\title{
16. X-RAY MINERALOGY STUDIES
}

\author{
R. W. Rex and B. Murray \\ University of California, Riverside, California
}

\section{INTRODUCTION}

The methods used in the X-ray mineralogy studies have been described briefly in the reports for Legs 1 and 2 and are discussed at greater length in Appendix III of Volume IV. The results of studies on core samples collected on Leg 5 of the Deep Sea Drilling Project are presented below.

\section{RESULTS}

\section{Site 32}

This site on the abyssal Delgada Fan provides a possible test area to compare California turbidite contributions with eolian and hydrogenous pelagic sediments. Recognition of these differences may be more complex than originally predicted.

Turbidites appear to be composed of a characteristic mixture that includes: K-feldspar, chlorite, mica, plagioclase, quartz and sometimes kaolinite. Calcite, montmorillonite, pyrite, zeolites, and amorphous material have no covariance within turbidites (Figure 1).

The pelagic lithogenous contribution is reflected in a close correlation of plagioclase with quartz as earlier reported by Rex and Goldberg (1962). It is notable that the plagioclase-quartz correlation (Figure 2) is not evident in some of the other Leg 5 holes because of volcanic plagioclase dilution. Further size studies are needed to see if the plagioclase-quartz correlation applies only to the aerosol-size range ( 1 to 20 microns), or to the coarser than 20-micron fraction which is attributed primarily to turbidity current transport. Pending resolution of this question the use of the quartz-plagioclase covariance as an indicator of eolian origin should be considered as only tentative.

Abundant K-feldspar seems to be restricted to the obviously turbidite sediments of both Plio-Pleistocene and Oligocene age. Montmorillonite is abundant in the Oligocene and Lower Miocene. The drop in montmorillonite abundance seems to be correlated with the lower-middle Miocene boundary in this area. The general scarcity of calcite implies a continuous depth below the calcium carbonate compensation depth. Chlorite abundances are difficult to interpret. In part, chlorite appears to be tied to turbidites and may serve as an indicator of uplift of California coastal metamorphic rocks such as the Franciscan series. Its absence during most of the Miocene might be indicative of entrapment by near coastal basins that could block seaward movement of turbidity currents. However, eolian chlorite is important in this part of the Pacific (Ferguson et al., 1970), and its presence or absence may also reflect changes of weathering style in the source areas.

The dolomite reported in the shipboard core description was not found in the X-ray diffraction analyses, even though careful analyses were performed to try to detect its presence. It is assumed that the sample coverage was insufficient to include the dolomitic basal portion of the Oligocene.

\section{Site 33}

The core at Site 33 is from the upper slope of an abyssal hill, westward of the base of Delgada Fan. Plagioclase feldspars are exceedingly abundant in this sediment while K-feldspars are of lesser importance, compared to Site 32. Quartz is relatively low in abundance, and clay minerals are of major importance. Mica is the dominant clay mineral followed by chlorite and kaolinite. Occasionally, montmorillonite-rich zones occur throughout the section, but montmorillonite, in general, is sparse except in the Middle Miocene. The montmorillonite appears to be associated with ashy zones. The pyrite reported in the shipboard analysis constitutes from 1 to 5 per cent of the $>20$-micron fraction of occasional samples throughout the cores. Pyrite usually consists of fillings and replacements of siliceous tests. The abundance of pyrite in the bulk samples is always less than 1 per cent; consequently, it is not listed in Figure 3. The general close correlation of quartz and plagioclase is suggestive that the quartzplagioclase correlation seen in eolian pelagic sediments may have been valid in most of Tertiary time. The mineralogy of this area is remarkably similar to the composition of modern aerosol dust reported in this area by Ferguson et al. (1970). No chert fragments were available for X-ray analysis.

\section{Site 34}

Site 34 is close to Site 33 and lies in a small abyssal plain adjacent to the abyssal hill flank location of Site 33 . The sediment assemblage is characteristically olive, gray or blue-gray mud containing mainly claysized material. The gross mineral assemblage is very similar to that observed in Site 33, except that pyrite 
is much more abundant especially in the Lower Pliocene and Middle Miocene of Site 34 (Figure 4). The amorphous content increases with age, being higher in Miocene sediments. The nannoplankton oozes are reflected by high calcite concentrations. The quartz and plagioclase are consistently covariant suggestive of an eolian contribution, while the mica, kaolinite and chlorite all show independent responses. Chlorite seems to be below detection limits from most of the Lower Miocene and Upper Oligocene section, whereas it was not seen in the Middle Miocene in Site 33. Sporadic occurrences of kaolinite appear in zones in the Miocene and Pliocene, and it is abundant in the Pleistocene. Kaolinite was not seen in the Lower Miocene and Upper Oligocene.

What appears to be diagenetic chlorite occurs at the bottom of the hole near the basaltic basement rock. The zones close to basement contact contain appreciable quantities of dolomite and clinoptilolite. Montmorillonite is absent in the Pleistocene, but is relatively abundant throughout the remainder of the hole. It is the most abundant mineral of several ashy zones of the Lower Miocene and Upper Oligocene.

\section{Site 35}

Site 35 is located in the western part of the Escanaba Trough, thought to be the active spreading center for the East Pacific Rise in this area. The assemblage consists almost entirely of turbidite-type materials with a banding suggestive of rhythmic varving (Figure 5). Detailed consideration of the mineralogy suggests minor mineralogic differences between the various layers, which would argue against their being artifacts resulting from multiple sampling of the same zone as a function of oscillation of the core barrel. The sediment assemblage is nearly mineralogically uniform throughout, reflecting primarily variations in the abundance of calcareous fossils and particle sizes. The fine fractions are richer in clay minerals, and the coarse fractions richer in plagioclase and quartz. Dolomite is common but not abundant from Cores 6 through 14. It is absent above and below these intervals. The abundance of kaolinite, chlorite and mica is suggestive that the Escanaba Trough has been receiving considerable quantities of continental sediments throughout the interval sampled by this drill hole. Quartz and plagioclase do not show obvious covariance suggesting that the lithogenous material is not primarily eolian.

\section{Site 36}

Site 36 lies in the abyssal hills region north of the Mendocino Fracture Zone. There appears to be a mixture of pelagic, continentally derived sediment in this suite of cores. The most striking mineralogic observation is that at 76 meters near the bottom of the hole, barite is abundant forming 3 to 6 per cent of the sediment (Figure 6). Barite occurs particularly in Cores 10 and 11 which show the greatest amount of reworked fossils.
Consequently, it may have been introduced by slumping from nearby upfaulted areas or it may be indigenous. Pyrite occurs as an infilling of fossils and worm tubes, and it is common in the Pleistocene and Pliocene. Montmorillonite is abundant in the Upper and Middle Miocene. Variations in plankton remains are reflected in the predominant inverse correlation between calcite and quartz (Figure 7).

Plagioclase and quartz show very close covariance suggestive of an eolian contribution (Figure 8). Occasionally some samples show anomalously abundant plagioclase, which is attributed to volcanic ash phenocrysts.

\section{Site 37}

Site 37 is located in the abyssal hills north of the Mendocino Fracture Zone. The sediments cored here contain round clay facies with various shades of coloring from yellow to dark brown. The sediments from Cores 1 and 2 contain abundant barite in a zone that contained reworked fossils (Figure 9). This site, which is considerably west of Site 36 but close to the Mendocino Fracture Zone, shows a barium enrichment similar to that reported in Site 36. The quartz-plagioclase relationship is widely scattered and suggests a divergent and variable source for the feldspars (Figure 10). The absence of plagioclase from quartz-rich sediments and their non-covariance suggest considerable contribution of feldspar from pyroclastic sources. The principal zeolite in this area is phillipsite, which was found in the Lower Pliocene (?) of Cores 2 and 3.

The most mineralogically striking observation is that the lower portion of Core 4 consists entirely of X-ray amorphous material. There is some suggestion of hematite in the diffraction pattern from this zone, but the majority of the sediment consists of mixed manganese and iron-hydrated oxides. It is suggested that this amorphous zone is mineralogically similar to the basal amorphous iron zone reported from the bottom of Hole 9A from the Atlantic.

\section{Site 38}

Site 38 is located due south of Site 37 and lies between the Mendocino and the Pioneer Fracture Zones. Cores consist of highly zeolitic "red" clay. The shallow clinoptilolite-containing zone reported in the core description was not provided for X-ray analysis. Phillipsite is extremely abundant constituting the entire fraction in some portions of Cores 3 and 4 (Figure 11). Core 2 is much more typical of a "red" clay and shows little covariance between quartz and plagioclase. Mica is the most abundant clay mineral, being more common than quartz. Site 38 is characterized by a nearly completely amorphous basal facies, possibly of Lower Tertiary age, which is diluted by variable amounts of calcite derived from nannoplankton. A very chloritic zone occurs in 
Core 5, which is associated with abundant plagioclase and kaolinite. It is possible that this chlorite zone might be a massive turbidite deposit extending over a large area. This same marker was also noted in Site 39. It merits further investigation both for its stratigraphic and geochemical implications.

\section{Site 39}

Site 39 is located several hundred miles south of Site 38 and is a relatively short distance north of the Murray Fracture Zone. It is located in an area of abyssal hills having a relief of 40 to 200 meters. The two cores analyzed showed a relative similarity to the assemblage seen in Site 38, several hundred miles away. A phillipsite-rich zone occurring in the lower portion of Core 1 is very similar to that observed in Cores 3 and 4, Site 38 (Figure 12). A highly chloritic marker which occurs in the bottom of Core 1 is similar to that observed in Core 4 of Site 38 . The basal zone of Upper Paleocene age consists of amorphous iron and manganese oxide. This amorphous iron-oxide zone appears to constitute a basal facies overlying basement in this area.

There does not seem to be a systematic covariance between plagioclase and quartz in the sediments in this area (Figure 13) suggesting a dilution of eolian sediment by other sources.

\section{Site 40}

Site 40 is located several hundred miles south of Site 39 , midway between the Molokai and Murray Fracture Zones in a pond of sediment around the base of a large abyssal hill. The upper portion of the hole contains very phillipsite-rich "red" clay characterized by abundant kaolinite and mica, as well as, some quartz and plagioclase (Figure 14). The phillipsite is replaced by clinoptilolite in the bottom of Core 1 in a unit which is thought to be of Upper Eocene age. The clinoptilolite zone is separated from the phillipsite zone by an entirely amorphous unit. The sediment below the clinoptilolite zone is X-ray amorphous to the bottom of the hole. Siliceous oozes constitute the majority of the X-ray amorphous materials. The cherts from Cores 17, 18 and 19 were not available for X-ray analysis. There appears to be a general covariance between quartz and plagioclase in the Upper Eocene, but there is considerable scatter in the correlation at higher quartz concentrations (Figure 15).

\section{Site 41}

Site 41 is only a short distance from Site 40 . Abundant phillipsite occurs here in Core 2 (Figure 16). The remainder of the mineral assemblage consists of quartz, mica, plagioclase, kaolinite and chlorite with occasional montmorillonite. The phillipsite-rich zone in the Eocene appears to be primarily altered volcanic ash with abundant phillipsite and plagioclase and little or no other clay minerals. Montmorillonite is surprisingly low or absent in this high phillipsite zone in the Eocene of Core 2, while it is abundant when associated with phillipsite in Core 1.

\section{Site 42}

Site 42 is located in the abyssal hills between the Clarion and Cliperton Fracture Zones in the northern margin of the thick equatorial siliceous and carbonate sediment belt. The X-ray mineralogy of the Eocene to Oligocene cores is very simple, with calcite usually constituting more than 90 per cent of the crystalline components (Figure 17). The amorphous material at times reaches 100 per cent, reflecting the abundance of biogenous opal. Pumice fragments and ashy zones in the Lower Oligocene and Upper Miocene cause high values of plagioclase. Scattered quartz zones in the Upper Eocene, Lower Oligocene and Upper Oligocene are suggested to be quartz possibly formed by the recrystallization of opal to chert.

A second adjacent hole, Hole 42.1, contained calcite and amorphous siliceous ooze of Middle Miocene age, but no other minerals in sufficient abundance to be recognized in the bulk samples (Figure 18).

\section{Site 43}

Site 43 is near the outer slope of the Hawaiian Arch in a depression close to abyssal hills. The cores show a greater mineralogical complexity than those encountered in Site 42, possibly because of the lesser dilution by biogenous ooze. Appreciable quantities of chlorite, kaolinite, quartz, plagioclase and montmorillonite were encountered (Figure 19). The chloritic zone of possible Middle Miocene age is suggestive of the chlorite-rich sediments found further to the northwest. Montmorillonite and plagioclase are probably of volcanic origin and constitute the main constituents of the sediments. The shortness of the cored interval make it difficult to try to correlate this section with holes further to the east.

\section{CONCLUSIONS}

The occurrences of a basal amorphous iron-oxide facies in cores from Sites 37, 38 and 39 are highly suggestive that sea floor spreading centers in both the Atlantic and Pacific emit iron- and manganese-rich solutions for a period of time after the initial eruption of the sea floor basalts. The significance of this sediment type is discussed elsewhere in this report. The occurrence of possible chloritic correlation marker zones associated with the Middle Tertiary deep sea sediment record is suggested in this leg. Detailed clay mineralogical studies should prove fruitful in helping to distinguish turbidite facies from pelagic sediment facies, such as, the eolian 
"red" clays of the Central Pacific. The stability of chlorite in sea water and possible diagenetic formation of chlorite may be fruitfully studied in the clays of Leg 5 .

\section{COMMENTS}

The X-ray diffraction samples for Leg 5 were reduced in size to 20 per cent of their normal volume through a shipboard oversight. Consequently, it was rarely possible to prepare more than a bulk sample for analysis. Systematic cross checks were made among the bulk $\mathrm{X}$-ray, shipboard descriptions and wet smear slides of the coarser than 20-micron fraction. Where all three sets of data are available, the authors observed lower zeolite concentrations than reported aboard ship, and, generally, noted an underestimation of calcite, quartz and amorphous matter with an overestimation of those phases which occur as large discrete crystals, grains or flakes. This appears to be a common error for numerous workers, including the authors, and should be considered in evaluating data in this report. The use of different samples from nearby sections of cores obviously may cause appreciable differences in quantitative results. Consequently, the majority of small discrepancies are thought to be within the noise range for the data reported. The larger discrepancies, where the X-ray data have been cross checked, are thought to be the results of sampling differences.

\section{REFERENCES}

Ferguson, W. S., Griffin, J. J. and Goldberg, E. D., 1970. Atmospheric dusts from the North Pacifica short note on long range eolian transport. $J$. Geophys. Res. 75, 1137.

Rex, R. W. and Goldberg, E. D., 1962. Insolubles. In The Sea. M. N. Hill (Ed.). New York (Interscience) $1,295$. 


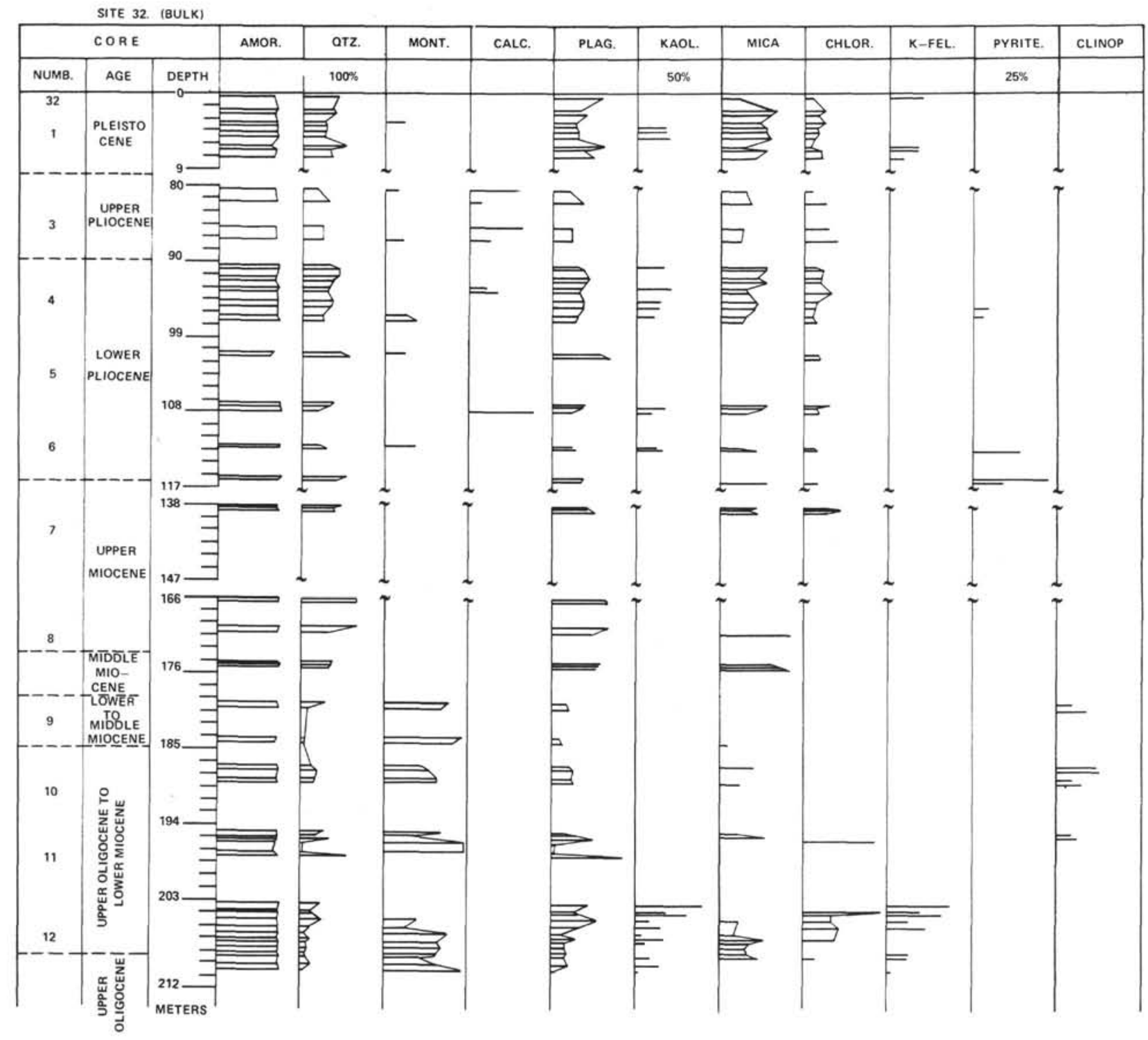

Figure 1. Site 32. (BULK) 


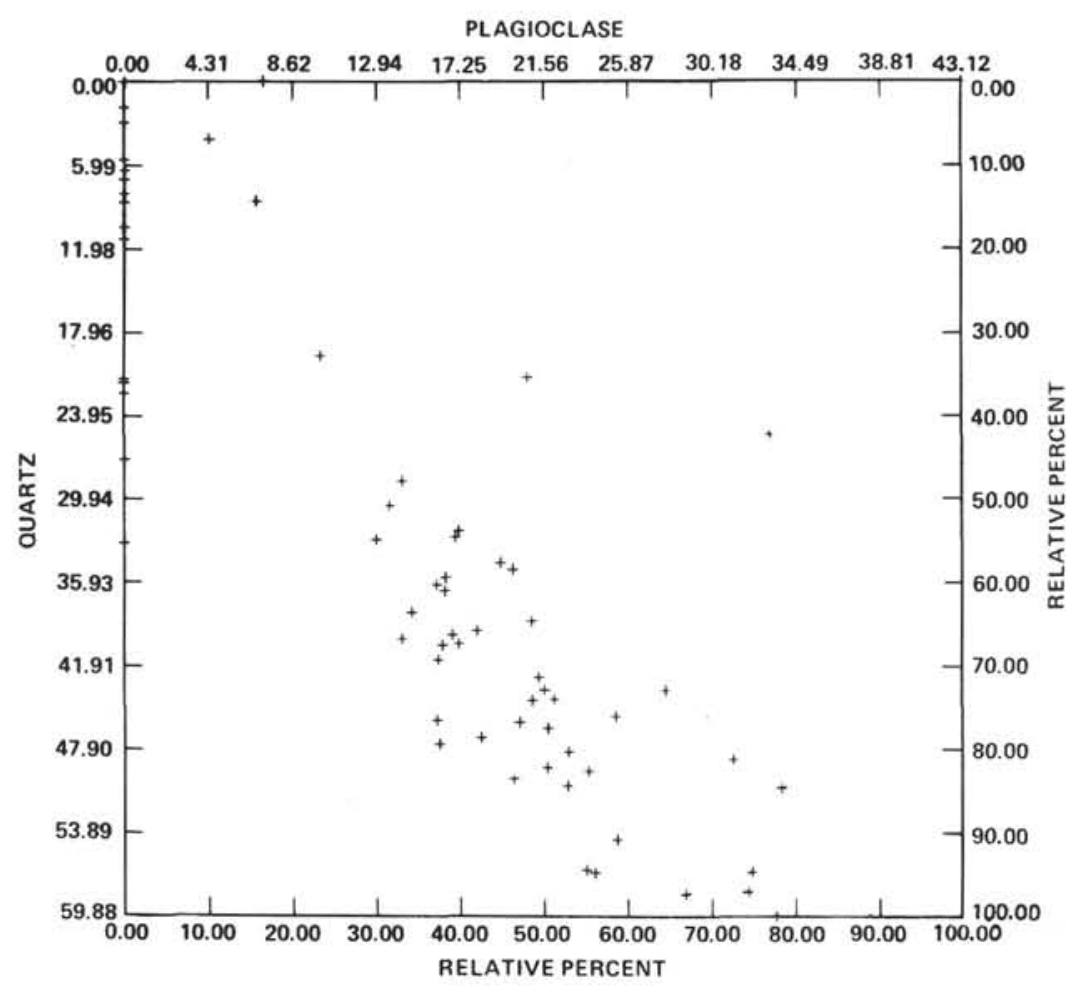

Figure 2. Site 32. Bulk Sample 4/3/70. 


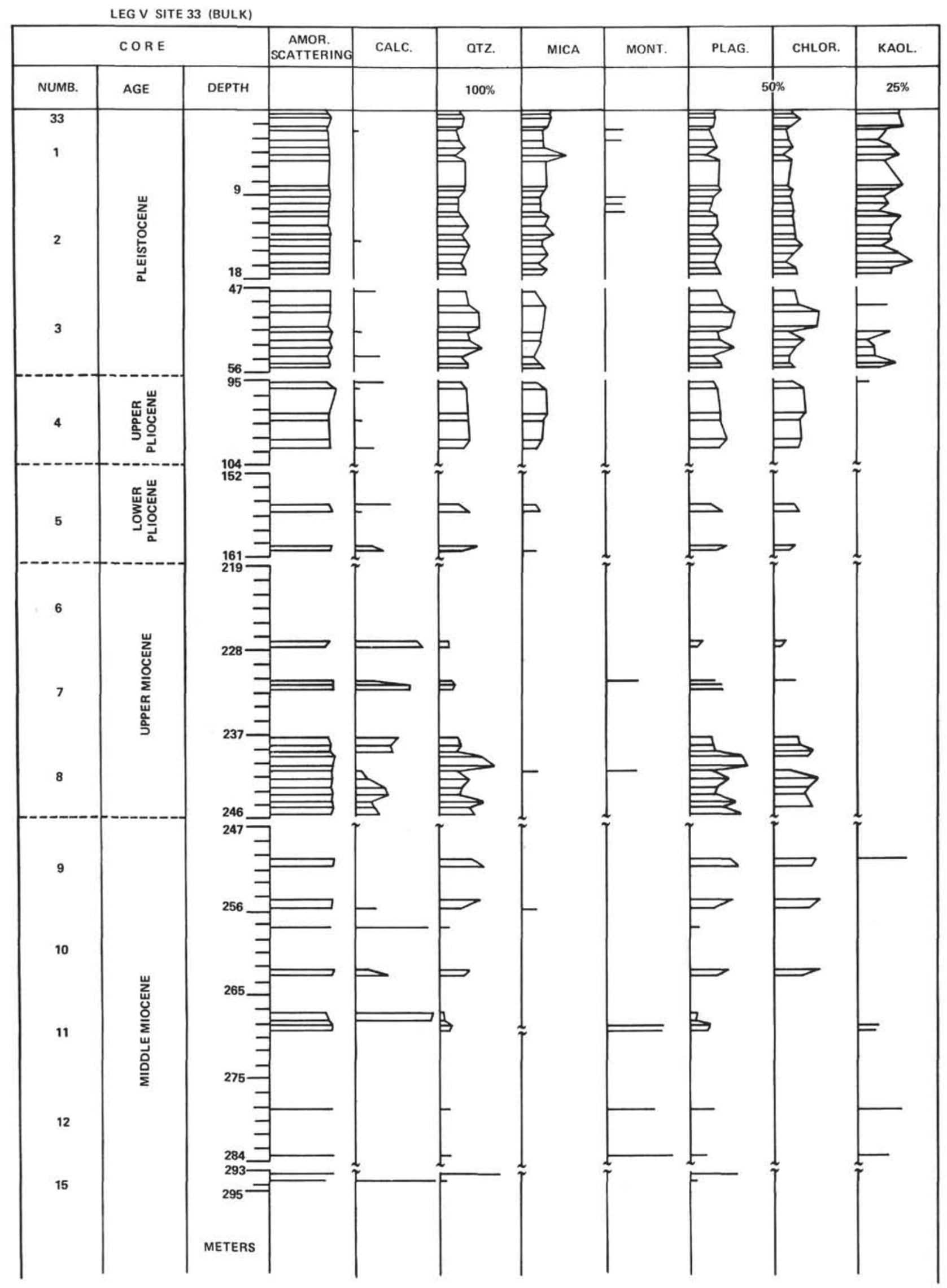

Figure 3. Site 33. (BULK) 
LEG V. SITE 34. (BULK)

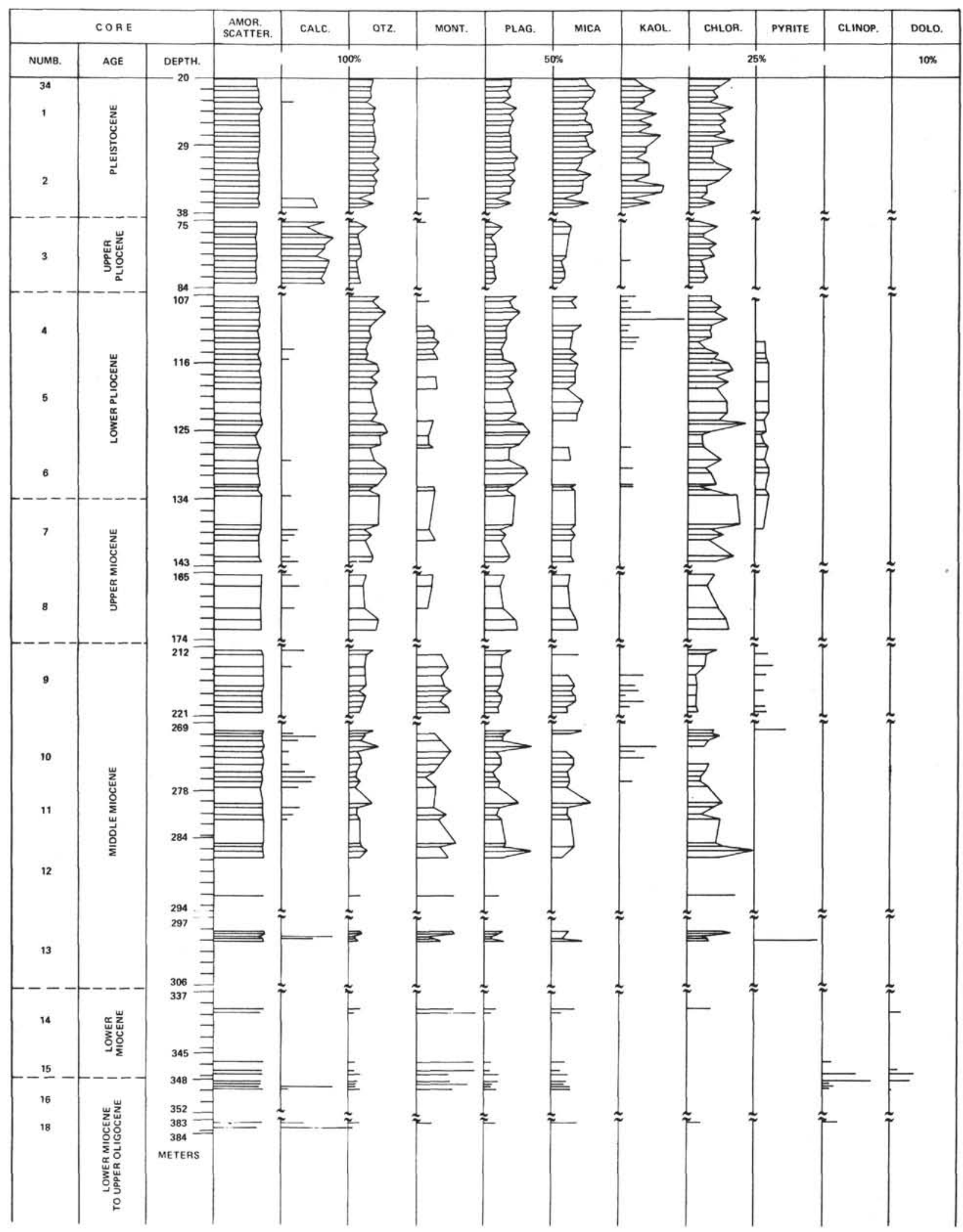

Figure 4. Site 34. (BULK) 


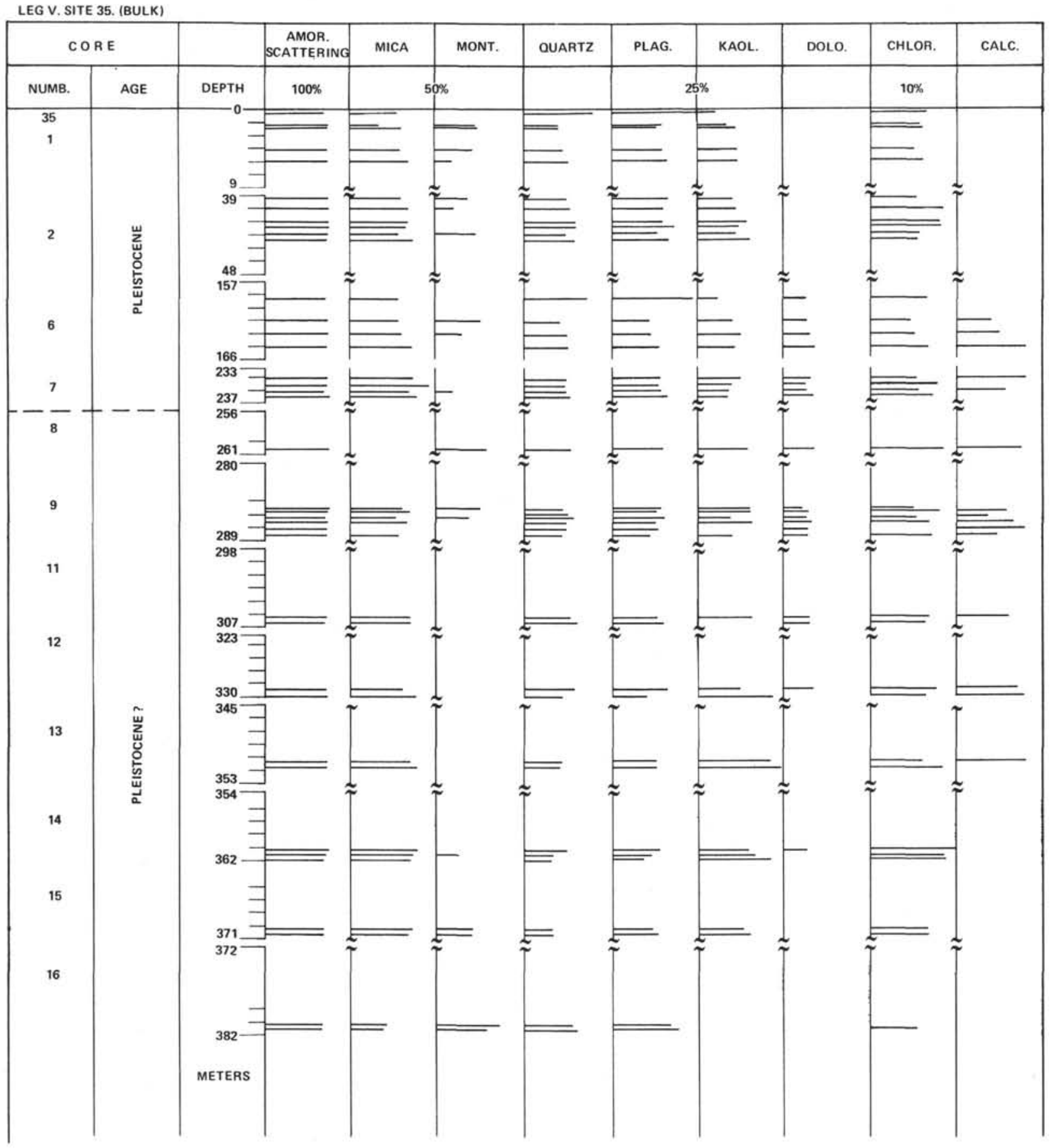

Figure 5. Site 35. (BULK) 


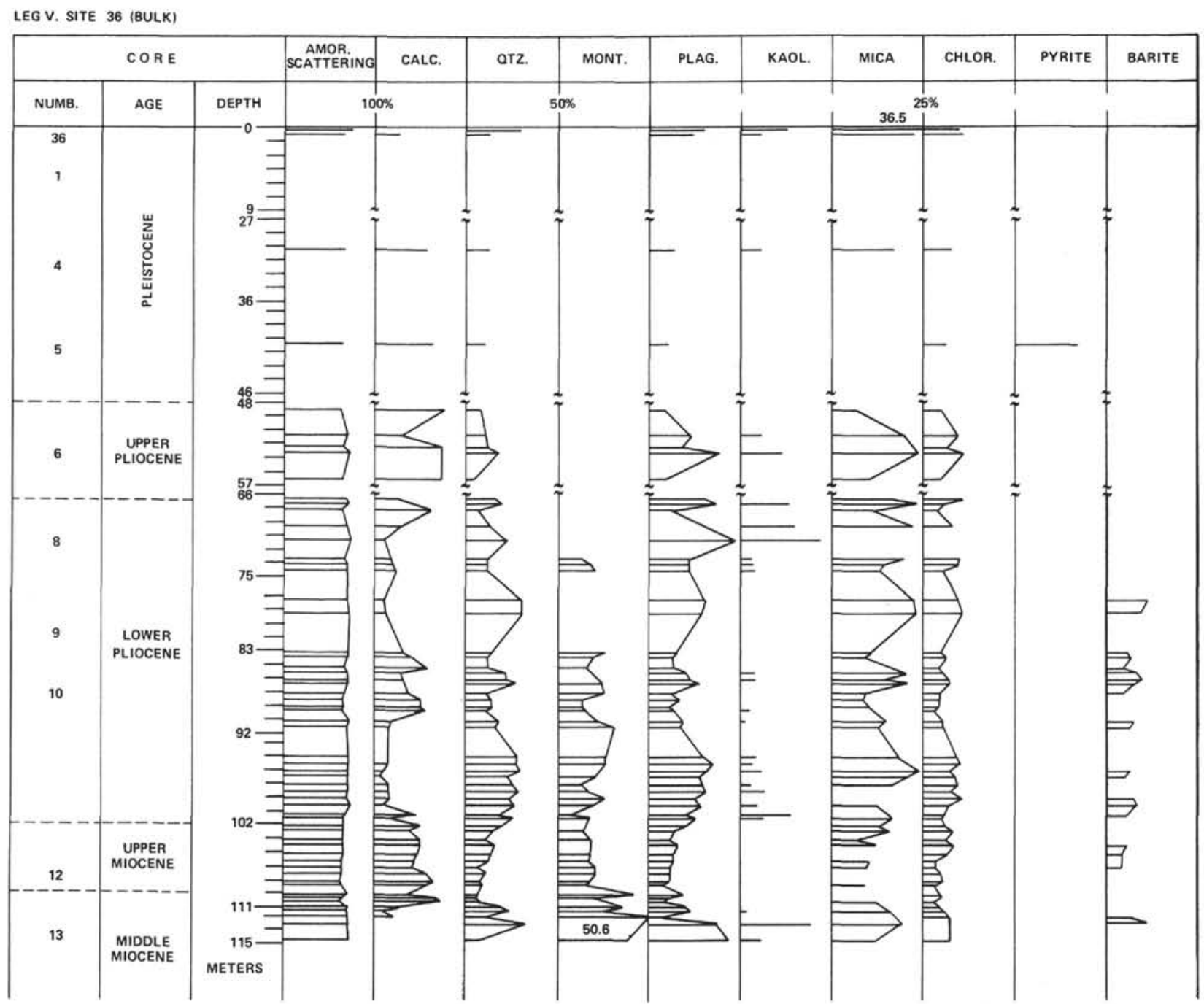

Figure 6. Site 36. (BULK) 


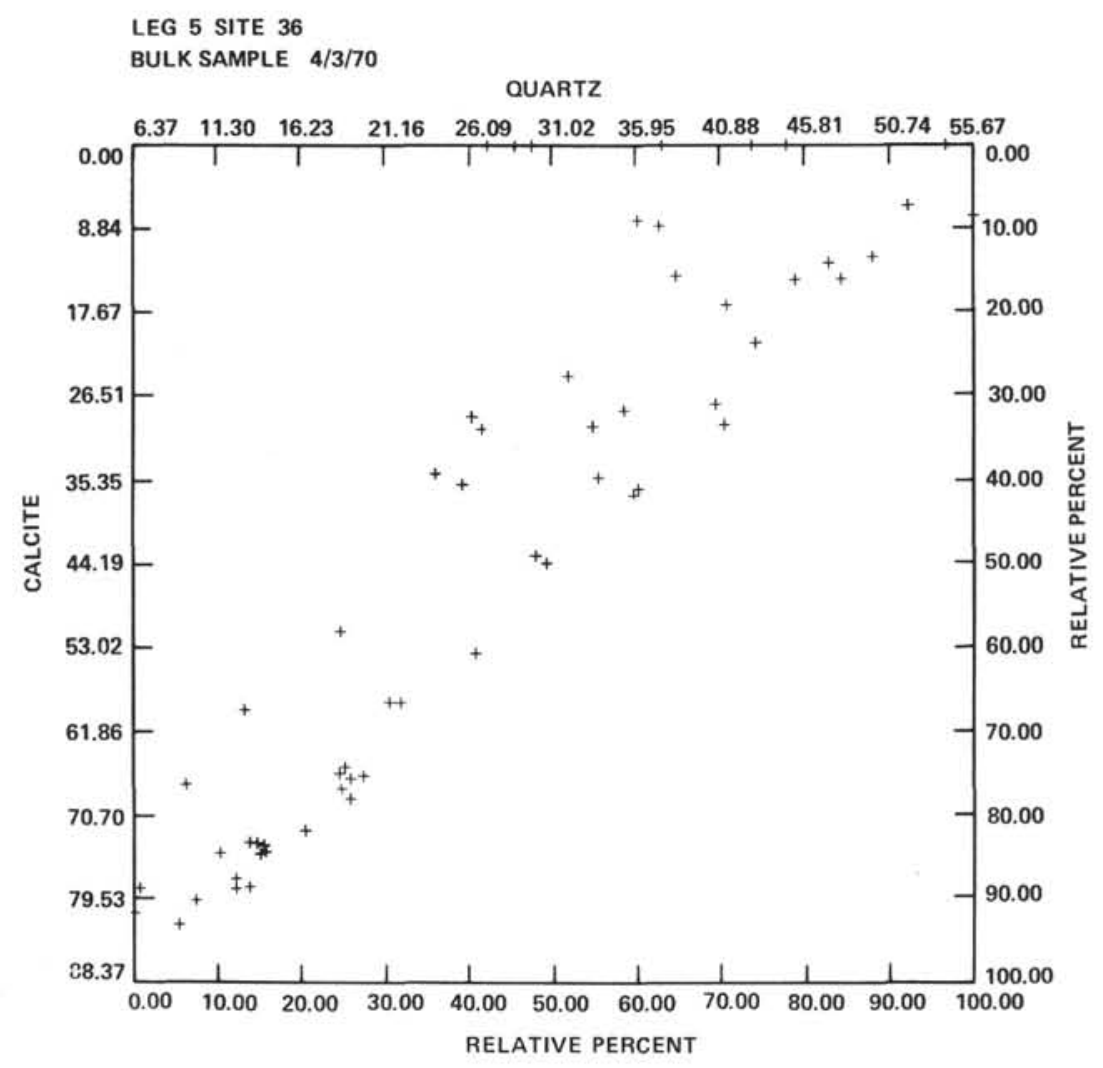

Figure 7. Site 36. Bulk Sample 4/3/70. 


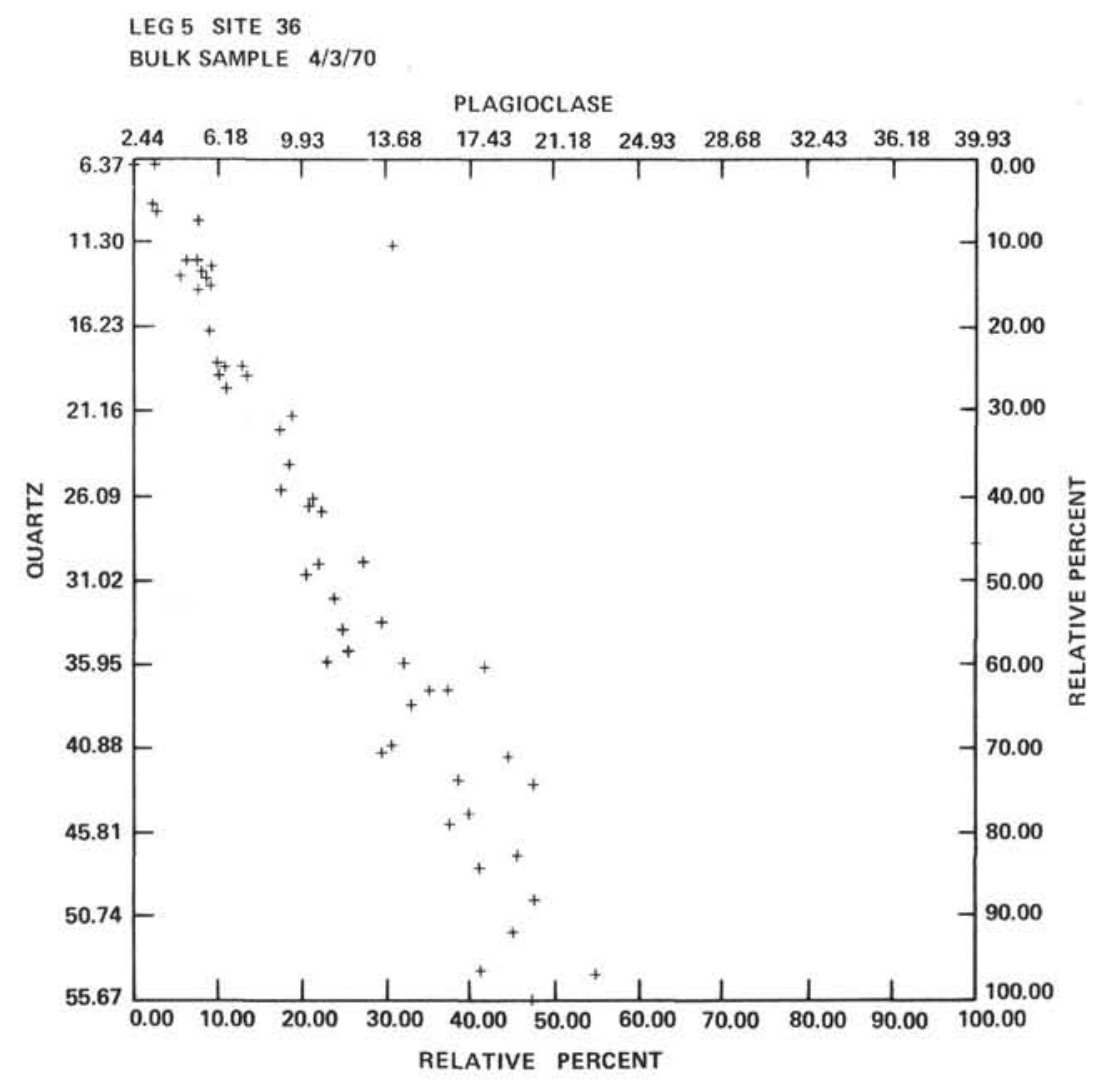

Figure 8. Site 36. Bulk Sample 4/3/70. 
LEG V. SITE 37 (BULK)

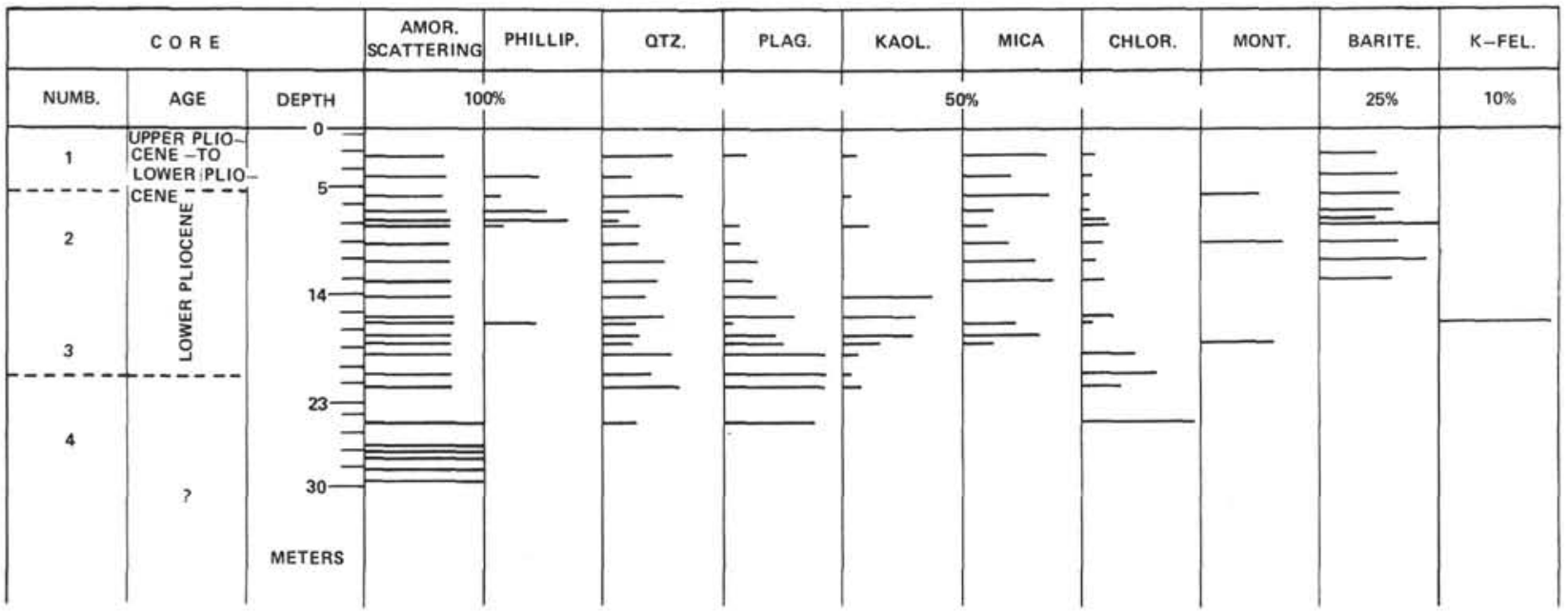

Figure 9. Site 37. (BULK)

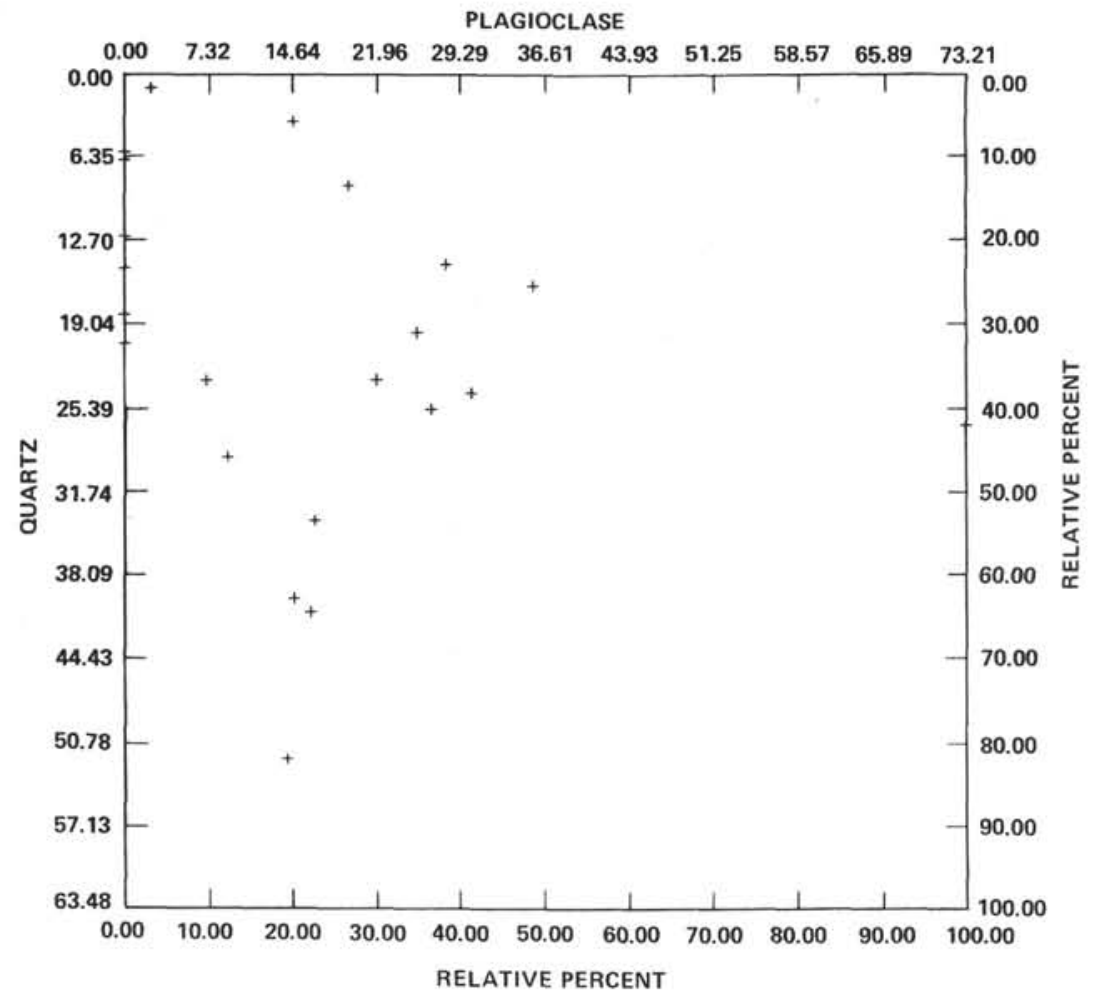

Figure 10. Site 37. Bulk Sample 4/3/70. 


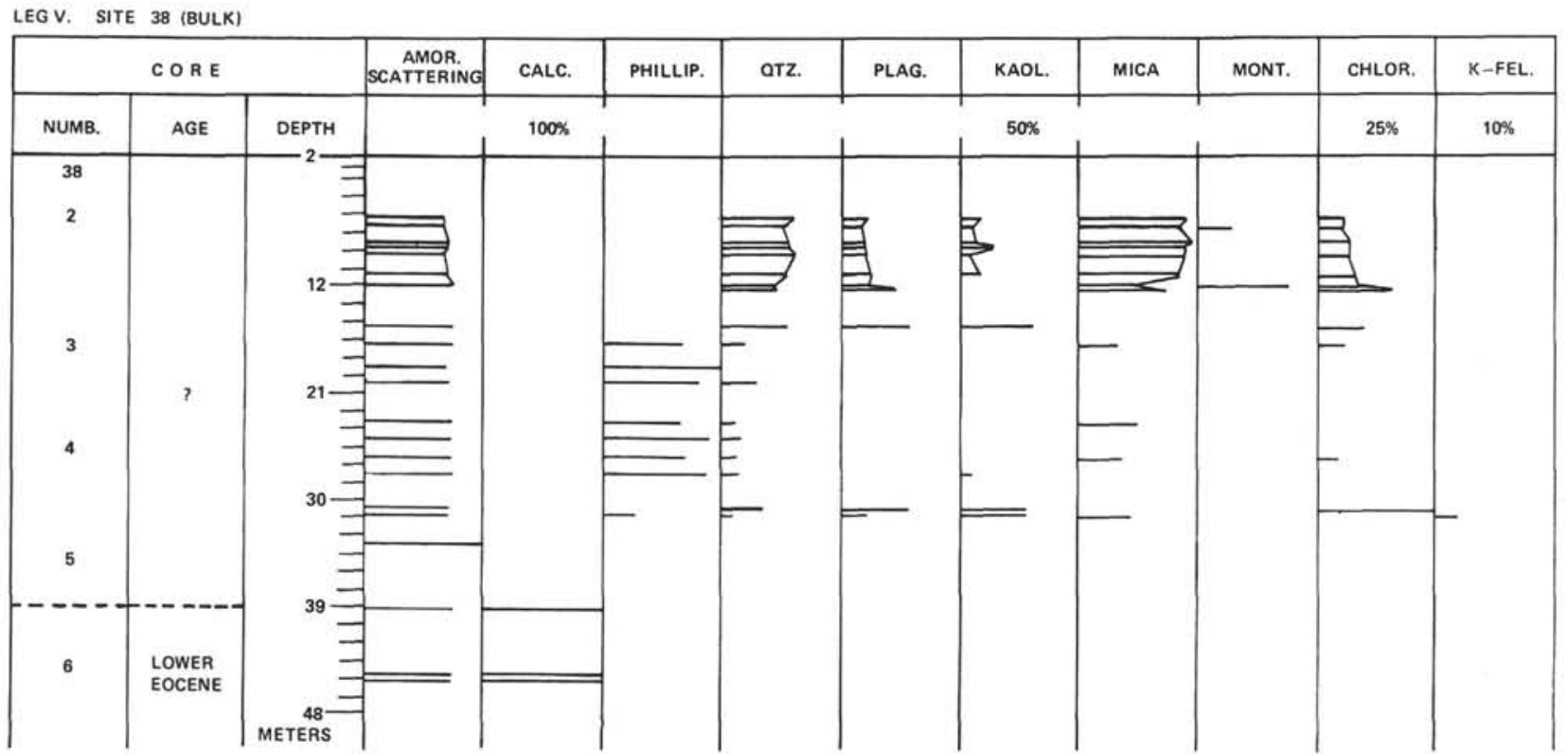

Figure 11. Site 38. (BULK)

LEG V. SITE 39 (BULK)

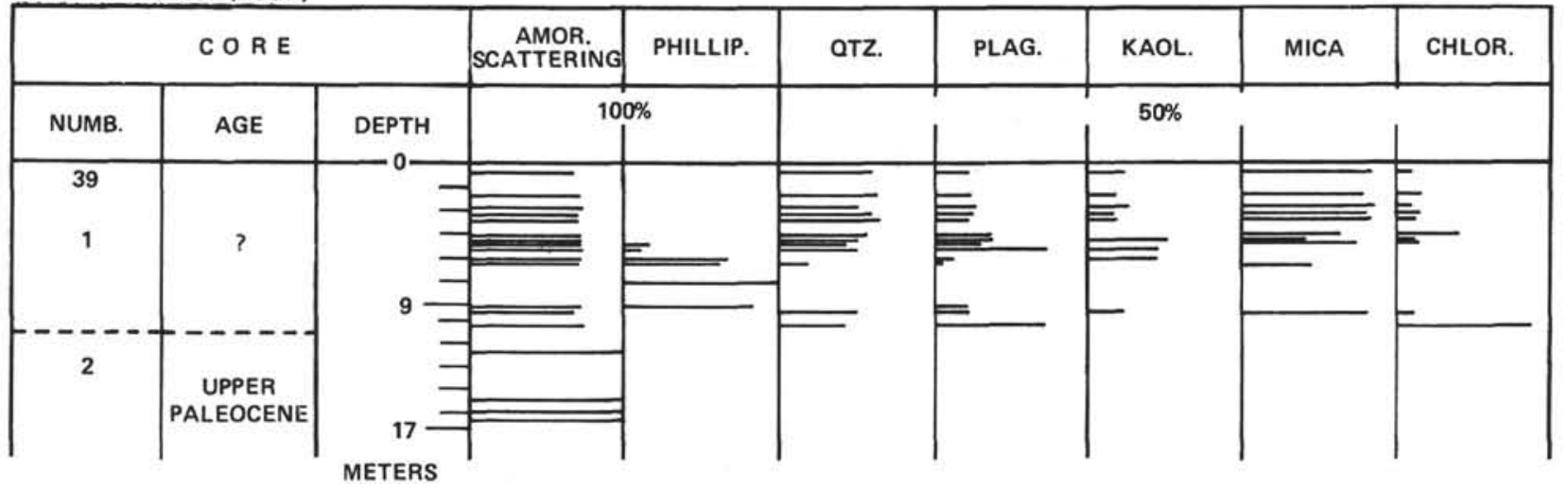

Figure 12. Site 39. (BULK) 


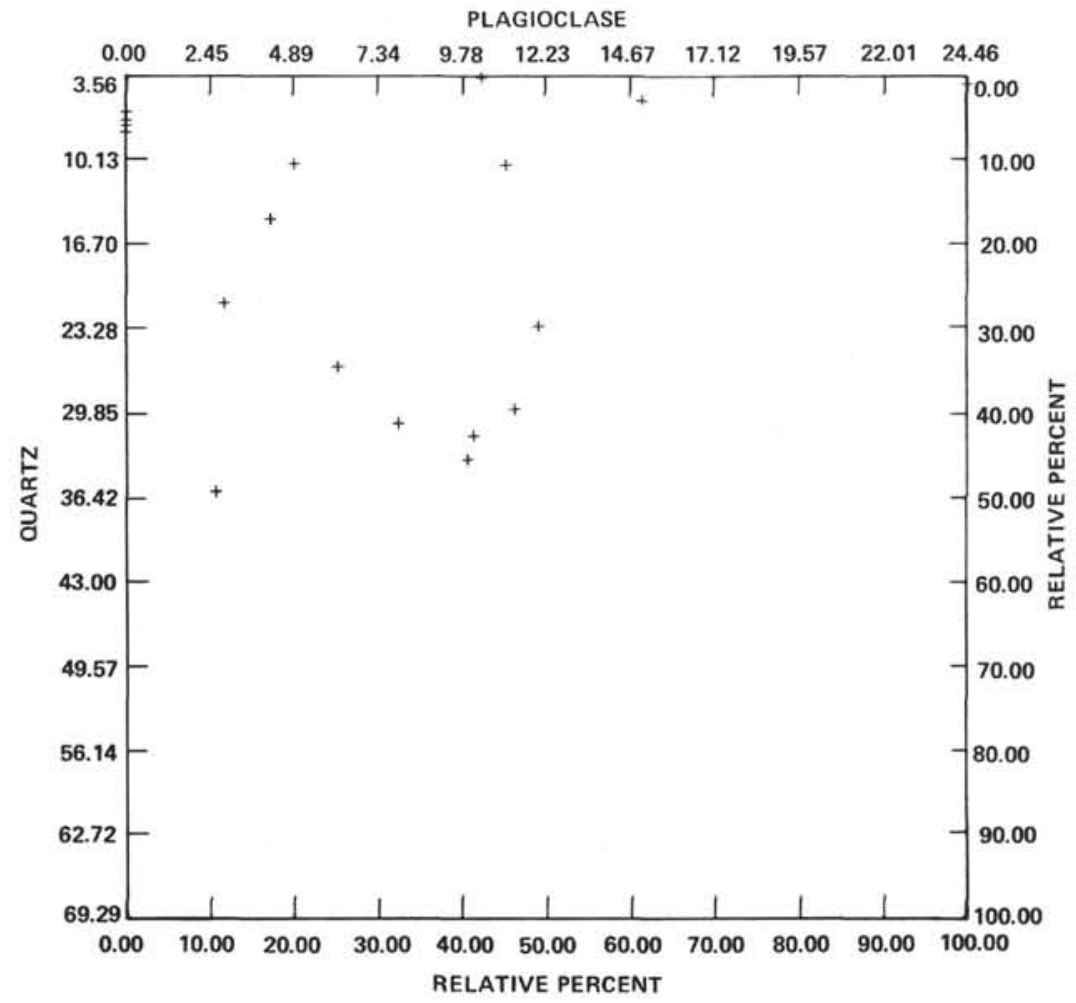

Figure 13. Site 39. Bulk Sample 4/3/70. 


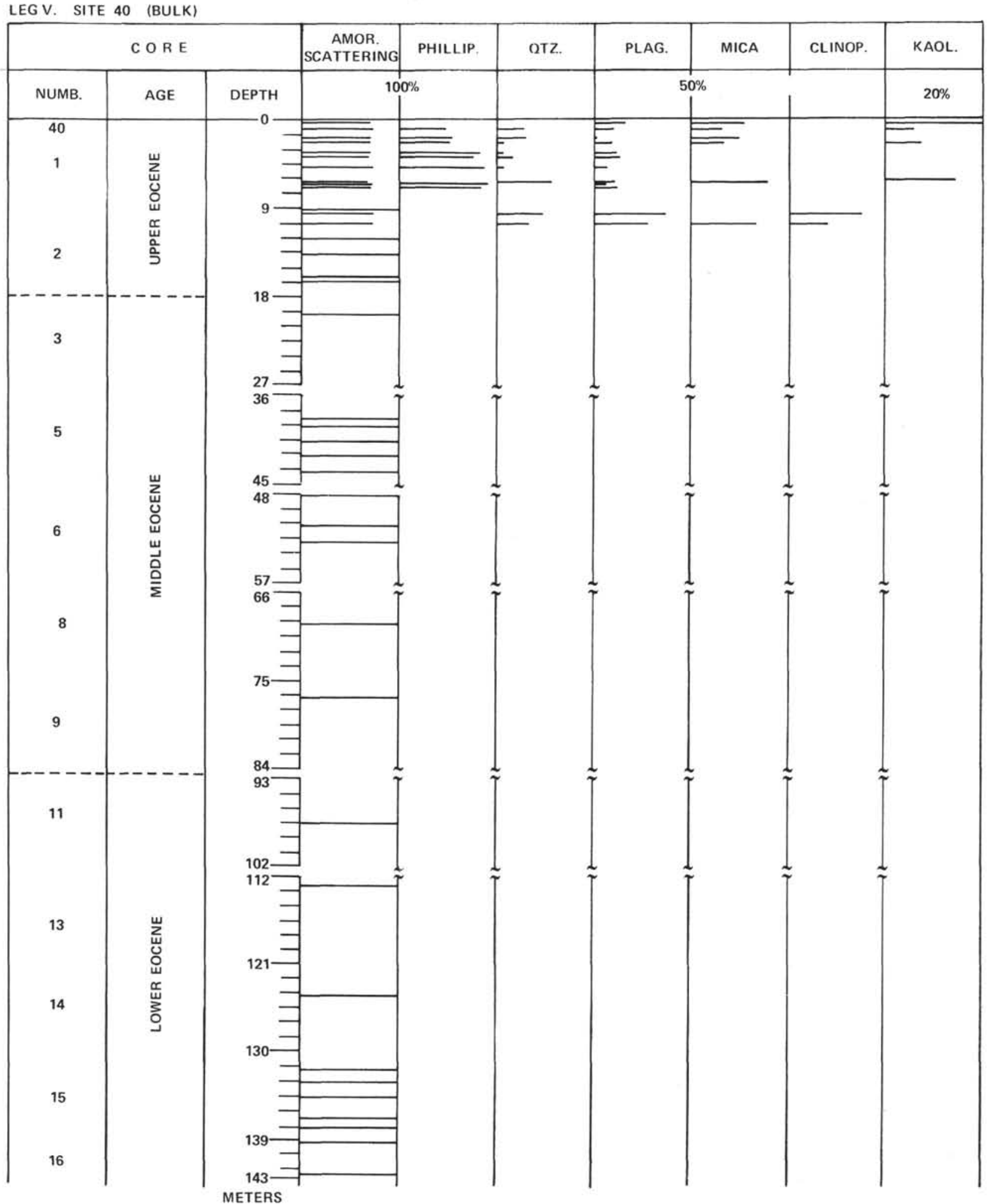

Figure 14. Site 40. (BULK) 


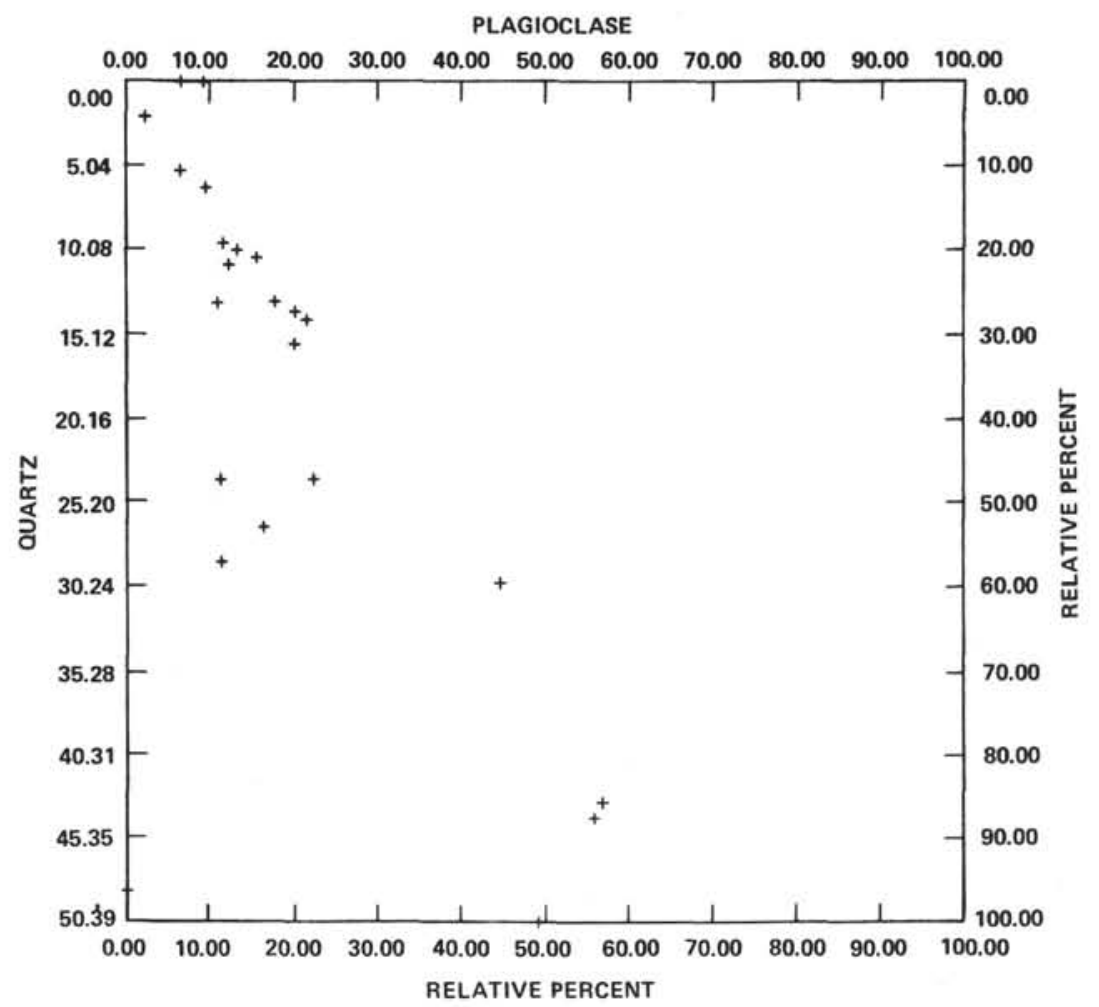

Figure 15. Site 40. Bulk Sample 4/3/70.

LEG V. SITE 41 (BULK)

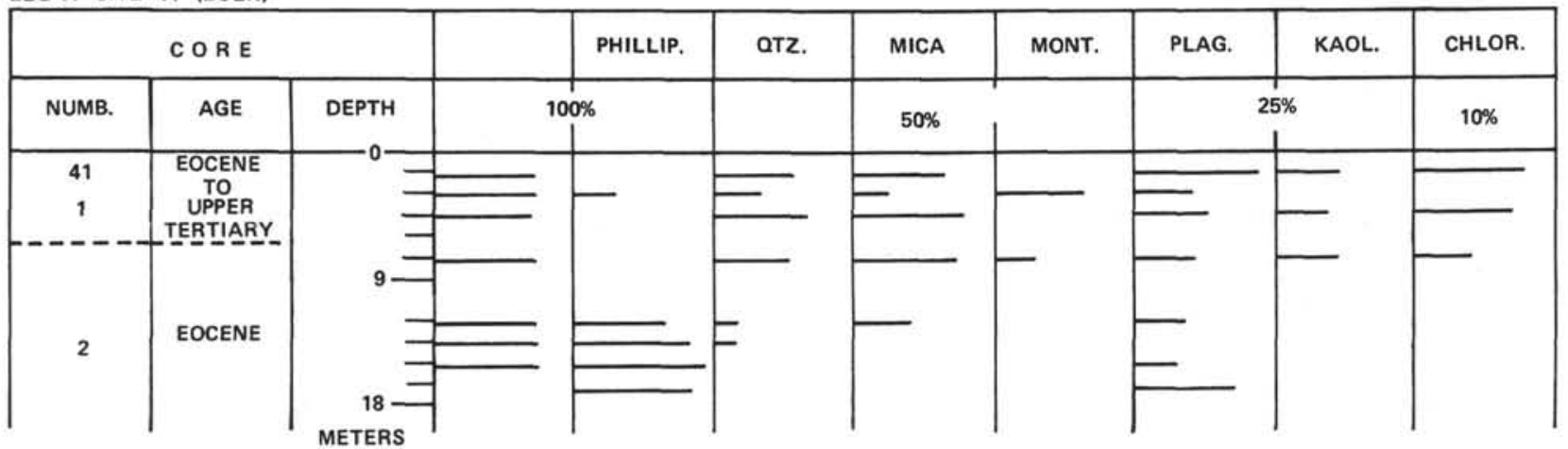

Figure 16. Site 41. (BULK) 


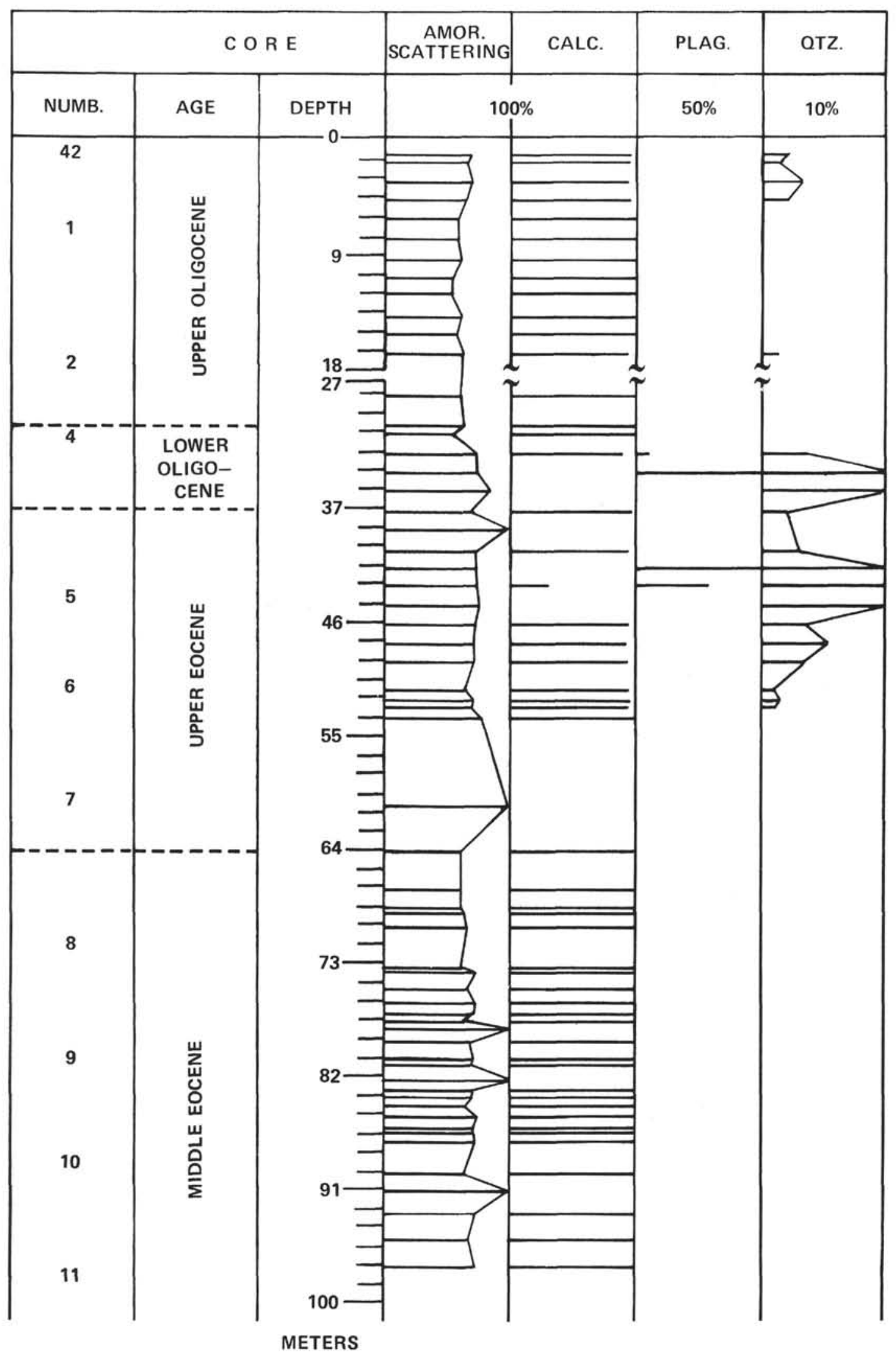

Figure 17. Site 42. (BULK) 


\begin{tabular}{|c|c|c|c|c|}
\hline \multicolumn{3}{|c|}{ CORE } & $\begin{array}{l}\text { AMOR. } \\
\text { SCATTERING }\end{array}$ & CALC. \\
\hline NUMB. & AGE & DEPTH & 100 & \\
\hline 42.1 & & & 默 & \\
\hline 1 & MIDDLE & & & \\
\hline & & $\begin{array}{c}107- \\
\text { METERS }\end{array}$ & & \\
\hline
\end{tabular}

Figure 18. Site 42.1. (BULK)

LEG V. SITE 43. (BULK)

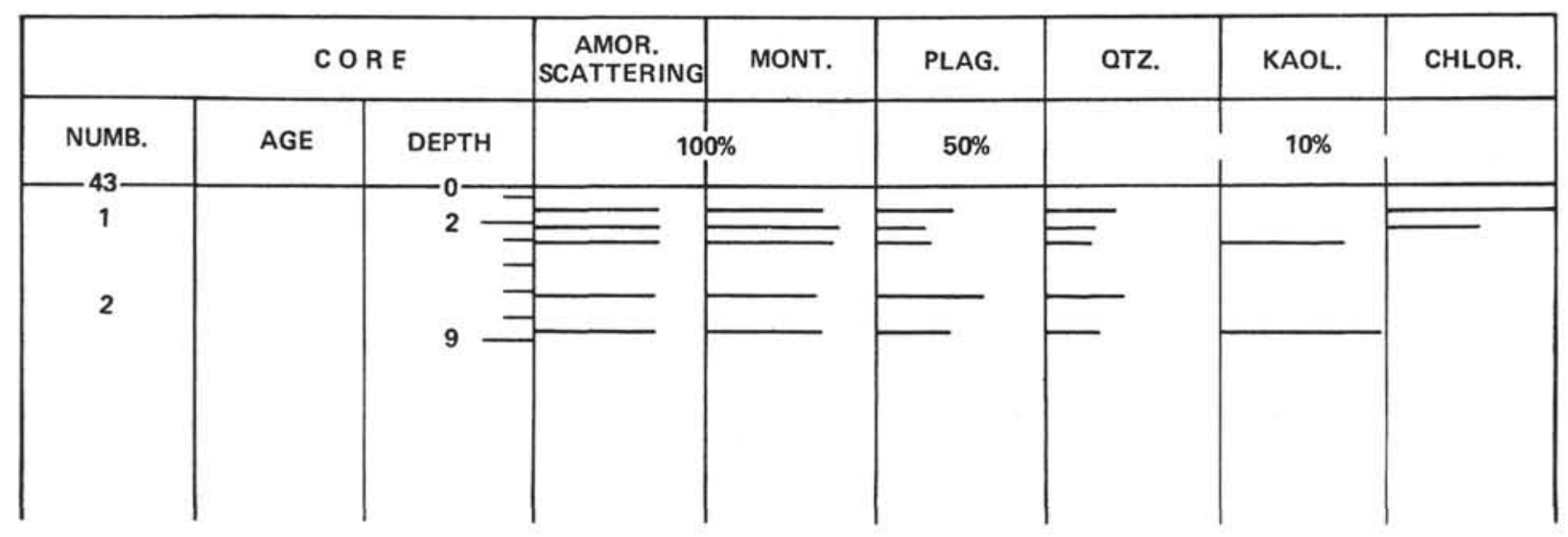

Figure 19. Site 43. (BULK) 
Results of X-Ray Diffraction Analysis of Bulk Samples from Leg 5

Hole 32

\begin{tabular}{|c|c|c|c|c|c|c|c|c|c|c|c|c|c|c|c|c|c|}
\hline Core & Section & $\begin{array}{l}\text { Depth } \\
\text { (cm) }\end{array}$ & Diff. & $\begin{array}{c}\% \\
\text { Amorphous }\end{array}$ & Calc. & Dolo. & Quar. & $\begin{array}{l}\text { K- } \\
\text { Feld. }\end{array}$ & Plag. & Kaol. & Mica & Chlo. & Mont. & Pyri. & Bari. & Clin. & Phil. \\
\hline 1 & 1 & $46-48$ & 89.4 & 68.8 & 00.0 & 00.0 & 44.0 & 10.1 & 30.4 & 00.0 & 10.6 & 4.8 & 00.0 & 00.0 & 00.0 & 00.0 & 00.0 \\
\hline 1 & 2 & NDG* & 89.9 & 70.3 & 00.0 & 00.0 & 36.4 & 00.0 & 15.3 & 00.0 & 34.9 & 13.4 & 00.0 & 00.0 & 00.0 & 00.0 & 00.0 \\
\hline 1 & 2 & NDG & 89.4 & 68.8 & 00.0 & 00.0 & 40.8 & 00.0 & 20.0 & 00.0 & 29.4 & 9.8 & 00.0 & 00.0 & 00.0 & 00.0 & 00.0 \\
\hline 1 & 3 & NDG & 90.1 & 70.9 & 00.0 & 00.0 & 28.4 & 00.0 & 14.5 & 00.0 & 23.3 & 13.6 & 20.2 & 00.0 & 00.0 & 00.0 & 00.0 \\
\hline 1 & 3 & $74-76$ & 89.8 & 70.0 & 00.0 & 00.0 & 30.5 & 00.0 & 14.9 & 17.1 & 29.3 & 8.2 & 00.0 & 00.0 & 00.0 & 00.0 & 00.0 \\
\hline 1 & 4 & $4-6$ & 90.0 & 70.6 & 00.0 & 00.0 & 29.5 & 00.0 & 15.0 & 17.7 & 28.3 & 9.4 & 00.0 & 00.0 & 00.0 & 00.0 & 00.0 \\
\hline 1 & 4 & $74-76$ & 90.3 & 71.5 & 00.0 & 00.0 & 29.2 & 00.0 & 15.2 & 18.7 & 31.3 & 5.6 & 00.0 & 00.0 & 00.0 & 00.0 & 00.0 \\
\hline 1 & 5 & $4-6$ & 87.5 & 63.2 & 00.0 & 00.0 & 52.1 & 00.0 & 31.2 & 00.0 & 12.5 & 4.2 & 00.0 & 00.0 & 00.0 & 00.0 & 00.0 \\
\hline 1 & 5 & $74-76$ & 89.6 & 69.4 & 00.0 & 00.0 & 34.5 & 8.6 & 19.0 & 00.0 & 28.3 & 10.0 & 00.0 & 00.0 & 00.0 & 00.0 & 00.0 \\
\hline 1 & 6 & $8-10$ & 89.1 & 67.1 & 00.0 & 00.0 & 37.9 & 8.0 & 25.0 & 00.0 & 18.2 & 11.0 & 00.0 & 00.0 & 00.0 & 00.0 & 00.0 \\
\hline 3 & 1 & $8-10$ & 89.3 & 68.5 & 33.2 & 00.0 & 18.6 & 4.3 & 9.6 & 00.0 & 15.2 & 5.9 & 13.2 & 00.0 & 00.0 & 00.0 & 00.0 \\
\hline 3 & 2 & 8.10 & 90.0 & 70.6 & 6.8 & 00.0 & 33.0 & 00.0 & 18.2 & 00.0 & 18.5 & 13.5 & 00.0 & 00.0 & 00.0 & 00.0 & 00.0 \\
\hline 3 & 4 & $8-10$ & 89.5 & 69.1 & 32.7 & 00.0 & 25.4 & 00.0 & 12.9 & 00.0 & 14.2 & 14.7 & 00.0 & 00.0 & 00.0 & 00.0 & 00.0 \\
\hline 3 & 5 & $8-10$ & 89.5 & 69.1 & 12.8 & 00.0 & 23.8 & 00.0 & 12.4 & 00.0 & 11.4 & 18.3 & 21.2 & 00.0 & 00.0 & 00.0 & 00.0 \\
\hline 4 & 1 & $10-12$ & 90.4 & 71.8 & 00.0 & 00.0 & 34.0 & 00.0 & 15.3 & 16.3 & 27.9 & 6.5 & 00.0 & 00.0 & 00.0 & 00.0 & 00.0 \\
\hline 4 & 1 & $74-76$ & 90.3 & 71.5 & 00.0 & 00.0 & 42.4 & 00.0 & 19.1 & 00.0 & 27.1 & 11.4 & 00.0 & 00.0 & 00.0 & 00.0 & 00.0 \\
\hline 4 & 2 & $4-6$ & 90.1 & 70.9 & 00.0 & 00.0 & 44.8 & 00.0 & 22.9 & 00.0 & 21.5 & 10.8 & 00.0 & 00.0 & 00.0 & 00.0 & 00.0 \\
\hline 4 & 2 & $74-76$ & 89.7 & 69.7 & 00.0 & 00.0 & 39.4 & 00.0 & 21.6 & 00.0 & 28.3 & 10.6 & 00.0 & 00.0 & 00.0 & 00.0 & 00.0 \\
\hline 4 & 3 & $4-6$ & 90.3 & 71.5 & 12.2 & 00.0 & 34.0 & 00.0 & 17.7 & 21.8 & 14.3 & 00.0 & 00.0 & 00.0 & 00.0 & 00.0 & 00.0 \\
\hline 4 & 3 & $74-76$ & 89.9 & 70.3 & 17.6 & 00.0 & 31.4 & 00.0 & 16.4 & 00.0 & 17.9 & 16.7 & 00.0 & 00.0 & 00.0 & 00.0 & 00.0 \\
\hline 4 & 4 & $15-17$ & 89.8 & 70.0 & 00.0 & 00.0 & 37.0 & 00.0 & 19.2 & 14.8 & 21.1 & 7.8 & 00.0 & 00.0 & 00.0 & 00.0 & 00.0 \\
\hline 4 & 4 & $118-120$ & 90.1 & 70.9 & 00.0 & 00.0 & 35.2 & 00.0 & 19.0 & 14.1 & 20.1 & 6.7 & 00.0 & 4.9 & 00.0 & 00.0 & 00.0 \\
\hline 4 & 5 & $20-22$ & 90.1 & 70.9 & 00.0 & 00.0 & 26.9 & 00.0 & 15.0 & 10.8 & 15.3 & 5.6 & 23.9 & 2.4 & 00.0 & 00.0 & 00.0 \\
\hline 4 & 5 & $90-92$ & 90.3 & 71.5 & 00.0 & 00.0 & 26.2 & 00.0 & 14.1 & 00.0 & 14.9 & 7.6 & 37.2 & 00.0 & 00.0 & 00.0 & 00.0 \\
\hline 5 & 2 & $12-13$ & 88.6 & 66.5 & 00.0 & 00.0 & 43.7 & 00.0 & 26.2 & 00.0 & 00.0 & 7.0 & 23.1 & 00.0 & 00.0 & 00.0 & 00.0 \\
\hline 5 & 2 & $73-75$ & 87.7 & 63.8 & 00.0 & 00.0 & 57.5 & 00.0 & 34.5 & 00.0 & 00.0 & 8.0 & 00.0 & 00.0 & 00.0 & 00.0 & 00.0 \\
\hline
\end{tabular}

*NDG $=$ No Data Given 
Results of X-Ray Diffraction Analysis of Bulk Samples from Leg 5 - Continued

Hole 32 - Continued

\begin{tabular}{|c|c|c|c|c|c|c|c|c|c|c|c|c|c|c|c|c|c|}
\hline Core & Section & $\begin{array}{l}\text { Depth } \\
(\mathrm{cm})\end{array}$ & Diff. & $\begin{array}{c}\% \\
\text { Amorphous }\end{array}$ & Calc. & Dolo. & Quar. & $\begin{array}{l}\text { K- } \\
\text { Feld. }\end{array}$ & Plag. & Kaol. & Mica & Chlo. & Mont. & Pyri. & Bari. & Clin. & Phil. \\
\hline 5 & 6 & $13-16$ & 90.7 & 72.6 & 00.0 & 00.0 & 37.3 & 00.0 & 19.8 & 00.0 & 27.6 & 15.3 & 00.0 & 00.0 & 00.0 & 00.0 & 00.0 \\
\hline 5 & 6 & $65-67$ & 90.8 & 72.9 & 00.0 & 00.0 & 32.6 & 00.0 & 17.6 & 16.9 & 26.7 & 6.2 & 00.0 & 00.0 & 00.0 & 00.0 & 00.0 \\
\hline 5 & 6 & $136-138$ & 91.0 & 73.5 & 37.6 & 00.0 & 19.2 & 00.0 & 10.4 & 9.4 & 14.5 & 8.8 & 00.0 & 00.0 & 00.0 & 00.0 & 00.0 \\
\hline 6 & 3 & $110-112$ & 90.9 & 73.2 & 00.0 & 00.0 & 20.1 & 00.0 & 11.0 & 11.2 & 10.5 & 5.4 & 35.7 & 00.0 & 00.0 & 00.0 & 00.0 \\
\hline 6 & 3 & $128-130$ & 91.0 & 73.5 & 00.0 & 00.0 & 29.1 & 00.0 & 13.4 & 15.1 & 21.5 & 7.8 & 00.0 & 13.1 & 00.0 & 00.0 & 00.0 \\
\hline 6 & 6 & $4-6$ & 91.5 & 75.0 & 00.0 & 00.0 & 53.8 & 00.0 & 24.2 & 00.0 & 00.0 & 00.0 & 00.0 & 22.0 & 00.0 & 00.0 & 00.0 \\
\hline 6 & 6 & $72-74$ & 90.8 & 72.9 & 00.0 & 00.0 & 35.6 & 00.0 & 16.0 & 00.0 & 29.2 & 9.6 & 00.0 & 9.6 & 00.0 & 00.0 & 00.0 \\
\hline 7 & 1 & $6-8$ & 90.4 & 71.8 & 00.0 & 00.0 & 45.0 & 00.0 & 20.7 & 00.0 & 22.1 & 12.2 & 00.0 & 00.0 & 00.0 & 00.0 & 00.0 \\
\hline 7 & 1 & $20-23$ & 90.1 & 70.9 & 00.0 & 00.0 & 39.8 & 00.0 & 22.7 & 00.0 & 16.3 & 21.1 & 00.0 & 00.0 & 00.0 & 00.0 & 00.0 \\
\hline 7 & 1 & $72-74$ & 90.7 & 72.6 & 00.0 & 00.0 & 40.6 & 00.0 & 25.2 & 00.0 & 23.2 & 11.0 & 00.0 & 00.0 & 00.0 & 00.0 & 00.0 \\
\hline 8 & 1 & $10-13$ & 91.0 & 73.5 & 00.0 & 00.0 & 67.6 & 00.0 & 32.2 & 00.0 & 00.0 & 00.0 & 00.0 & 00.0 & 00.0 & 00.0 & 00.0 \\
\hline 8 & 1 & $24-27$ & 91.1 & 73.8 & 00.0 & 00.0 & 66.5 & 00.0 & 33.5 & 00.0 & 00.0 & 00.0 & 00.0 & 00.0 & 00.0 & 00.0 & 00.0 \\
\hline 8 & 3 & $33-36$ & 91.5 & 75.0 & 00.0 & 00.0 & 66.0 & 00.0 & 34.0 & 00.0 & 00.0 & 00.0 & 00.0 & 00.0 & 00.0 & 00.0 & 00.0 \\
\hline 8 & 3 & $102-104$ & 91.2 & 74.1 & 00.0 & 00.0 & 34.4 & 00.0 & 23.4 & 00.0 & 42.2 & 00.0 & 00.0 & 00.0 & 00.0 & 00.0 & 00.0 \\
\hline 8 & 6 & $15-17$ & 90.9 & 73.2 & 00.0 & 00.0 & 38.9 & 00.0 & 29.2 & 00.0 & 31.9 & 00.0 & 00.0 & 00.0 & 00.0 & 00.0 & 00.0 \\
\hline 8 & 6 & $24-26$ & 91.3 & 74.4 & 00.0 & 00.0 & 36.6 & 00.0 & 27.5 & 00.0 & 35.9 & 00.0 & 00.0 & 00.0 & 00.0 & 00.0 & 00.0 \\
\hline 8 & 6 & $74-76$ & 91.0 & 73.5 & 00.0 & 00.0 & 33.6 & 00.0 & 25.2 & 00.0 & 41.2 & 00.0 & 00.0 & 00.0 & 00.0 & 00.0 & 00.0 \\
\hline 9 & 3 & $23-26$ & 90.8 & 72.9 & 00.0 & 00.0 & 27.3 & 00.0 & 9.2 & 00.0 & 00.0 & 00.0 & 77.8 & 00.0 & 00.0 & 5.6 & 00.0 \\
\hline 9 & 3 & $120-122$ & 91.2 & 74.1 & 00.0 & 00.0 & 9.8 & 00.0 & 10.6 & 00.0 & 00.0 & 00.0 & 69.8 & 00.0 & 00.0 & 9.8 & 00.0 \\
\hline 9 & 6 & $5-7$ & 90.7 & 72.6 & 00.0 & 00.0 & 3.6 & 00.0 & 4.6 & 00.0 & 00.0 & 00.0 & 91.8 & 00.0 & 00.0 & 00.0 & 00.0 \\
\hline 9 & 6 & $74-76$ & 89.9 & 70.3 & 00.0 & 00.0 & 4.5 & 00.0 & 6.8 & 00.0 & 5.4 & 00.0 & 80.3 & 00.0 & 00.0 & 00.0 & 00.0 \\
\hline 10 & 2 & $10-12$ & 90.7 & 72.6 & 00.0 & 00.0 & 12.4 & 00.0 & 10.3 & 00.0 & 20.4 & 00.0 & 44.3 & 00.0 & 00.0 & 12.4 & 00.0 \\
\hline 10 & 2 & $100-102$ & 90.9 & 73.2 & 00.0 & 00.0 & 20.0 & 00.0 & 13.4 & 00.0 & 00.0 & 00.0 & 53.4 & 00.0 & 00.0 & 13.2 & 00.0 \\
\hline 10 & 3 & $11-13$ & 90.7 & 72.6 & 00.0 & 00.0 & 18.0 & 00.0 & 12.1 & 00.0 & 00.0 & 00.0 & 64.0 & 00.0 & 00.0 & 5.9 & 00.0 \\
\hline 10 & 3 & $90-92$ & 90.9 & 73.2 & 00.0 & 00.0 & 17.4 & 00.0 & 13.1 & 00.0 & 12.6 & 00.0 & 62.0 & 00.0 & 00.0 & 7.5 & 00.0 \\
\hline
\end{tabular}


Hole 32 - Continued

\begin{tabular}{|c|c|c|c|c|c|c|c|c|c|c|c|c|c|c|c|c|c|}
\hline Core & Section & $\begin{array}{l}\text { Depth } \\
(\mathrm{cm})\end{array}$ & Diff. & $\begin{array}{c}\% \\
\text { Amorphous }\end{array}$ & Calc. & Dolo. & Quar. & $\begin{array}{l}\text { K- } \\
\text { Feld. }\end{array}$ & Plag. & Kaol. & Mica & Chlo. & Mont. & Pyri. & Bari. & Clin. & Phil. \\
\hline 11 & 1 & $89-91$ & 90.8 & 72.9 & 00.0 & 00.0 & 27.7 & 00.0 & 6.4 & 00.0 & 12.6 & 00.0 & 68.3 & 00.0 & 00.0 & 5.0 & 00.0 \\
\hline 11 & 1 & $139-141$ & 90.8 & 72.9 & 00.0 & 00.0 & 16.7 & 00.0 & 15.1 & 00.0 & 27.4 & 00.0 & 29.8 & 00.0 & 00.0 & 11.0 & 00.0 \\
\hline 11 & 2 & $15-17$ & 90.7 & 72.6 & 00.0 & 00.0 & 33.6 & 00.0 & 24.8 & 00.0 & 00.0 & 41.6 & 00.0 & 00.0 & 00.0 & 00.0 & 00.0 \\
\hline 11 & 2 & $78-82$ & 89.8 & 70.0 & 00.0 & 00.0 & 2.1 & 00.0 & 2.6 & 00.0 & 00.0 & 00.0 & 95.3 & 00.0 & 00.0 & 00.0 & 00.0 \\
\hline 11 & 3 & $26-28$ & 88.9 & 67.4 & 00.0 & 00.0 & 1.0 & 00.0 & 1.1 & 00.0 & 00.0 & 00.0 & 97.9 & 00.0 & 00.0 & 00.0 & 00.0 \\
\hline 11 & 3 & $74-76$ & 90.9 & 73.2 & 00.0 & 00.0 & 56.6 & 00.0 & 43.4 & 00.0 & 00.0 & 00.0 & 00.0 & 00.0 & 00.0 & 00.0 & 00.0 \\
\hline 12 & 1 & $45-47$ & 91.2 & 74.1 & 00.0 & 00.0 & 20.2 & 18.1 & 21.2 & 40.5 & 00.0 & 00.0 & 00.0 & 00.0 & 00.0 & 00.0 & 00.0 \\
\hline 12 & 1 & $98-100$ & 90.4 & 71.8 & 00.0 & 00.0 & 11.5 & 10.3 & 13.8 & 18.4 & 00.0 & 46.0 & 00.0 & 00.0 & 00.0 & 00.0 & 00.0 \\
\hline 12 & 2 & $5-7$ & 90.8 & 72.9 & 00.0 & 00.0 & 15.3 & 16.7 & 20.7 & 30.7 & 00.0 & 16.3 & 00.0 & 00.0 & 00.0 & 00.0 & 00.0 \\
\hline 12 & 2 & $75-77$ & 90.6 & 72.4 & 00.0 & 00.0 & 27.5 & 6.7 & 27.9 & 9.0 & 12.3 & 16.3 & 40.3 & 00.0 & 00.0 & 00.0 & 00.0 \\
\hline 12 & 3 & $9-11$ & 90.9 & 73.2 & 00.0 & 00.0 & 13.2 & 11.9 & 15.9 & 15.9 & 00.0 & 21.1 & 21.7 & 00.0 & 00.0 & 00.0 & 00.0 \\
\hline 12 & 3 & $90-92$ & 91.3 & 74.4 & 00.0 & 00.0 & 4.7 & 00.0 & 5.0 & 3.8 & 9.7 & 00.0 & 76.7 & 00.0 & 00.0 & 00.0 & 00.0 \\
\hline 12 & 4 & $7-9$ & 90.9 & 73.2 & 00.0 & 00.0 & 13.1 & 00.0 & 14.7 & 26.2 & 26.9 & 19.1 & 00.0 & 00.0 & 00.0 & 00.0 & 00.0 \\
\hline 12 & 4 & $20-22$ & 91.4 & 74.7 & 00.0 & 00.0 & 7.0 & 00.0 & 7.3 & 5.6 & 17.5 & 00.0 & 62.5 & 00.0 & 00.0 & 00.0 & 00.0 \\
\hline 12 & 4 & $124-126$ & 91.2 & 74.1 & 00.0 & 00.0 & 9.4 & 00.0 & 7.8 & 00.0 & 15.2 & 00.0 & 67.5 & 00.0 & 00.0 & 00.0 & 00.0 \\
\hline 12 & 5 & $20-22$ & 91.0 & 73.5 & 00.0 & 00.0 & 6.8 & 6.2 & 9.2 & 00.0 & 16.8 & 00.0 & 61.0 & 00.0 & 00.0 & 00.0 & 00.0 \\
\hline 12 & 5 & $74-76$ & 91.2 & 74.1 & 00.0 & 00.0 & 7.3 & 6.5 & 7.7 & 8.7 & 24.0 & 6.8 & 39.0 & 00.0 & 00.0 & 00.0 & 00.0 \\
\hline 12 & 6 & $20-22$ & 91.1 & 73.8 & 00.0 & 00.0 & 11.8 & 00.0 & 10.7 & 14.2 & 00.0 & 00.0 & 63.3 & 00.0 & 00.0 & 00.0 & 00.0 \\
\hline 12 & 6 & $74-76$ & 91.5 & 75.0 & 00.0 & 00.0 & 2.6 & 1.5 & 2.7 & 1.5 & 00.0 & 00.0 & 91.7 & 00.0 & 00.0 & 00.0 & 00.0 \\
\hline \multicolumn{18}{|c|}{ Hole 33} \\
\hline Core & Section & $\begin{array}{l}\text { Depth } \\
\text { (cm) }\end{array}$ & Diff. & $\begin{array}{c}\% \\
\text { Amorphous }\end{array}$ & Calc. & Dolo. & Quar. & $\begin{array}{l}\text { K- } \\
\text { Feld. }\end{array}$ & Plag. & Kaol. & Mica & Chlo. & Mont. & Pyri. & Bari. & Clin. & Phil. \\
\hline 1 & 1 & $4-6$ & 90.0 & 70.6 & 00.0 & 00.0 & 28.3 & 00.0 & 15.0 & 11.3 & 34.0 & 11.3 & 00.0 & 00.0 & 00.0 & 00.0 & 00.0 \\
\hline 1 & 1 & $86-88$ & 90.5 & 72.1 & 00.0 & 00.0 & 31.7 & 00.0 & 15.9 & 00.0 & 35.6 & 16.8 & 00.0 & 00.0 & 00.0 & 00.0 & 00.0 \\
\hline 1 & 2 & $5-6$ & 90.0 & 70.6 & 00.0 & 00.0 & 31.2 & 00.0 & 15.0 & 13.8 & 30.0 & 10.0 & 00.0 & 00.0 & 00.0 & 00.0 & 00.0 \\
\hline 1 & 2 & $80-81$ & 90.0 & 70.6 & 4.0 & 00.0 & 21.3 & 00.0 & 11.9 & 8.5 & 23.8 & 7.9 & 22.6 & 00.0 & 00.0 & 00.0 & 00.0 \\
\hline
\end{tabular}


Results of X-Ray Diffraction Analysis of Bulk Samples from Leg 5 - Continued

Hole 33 - Continued

\begin{tabular}{|c|c|c|c|c|c|c|c|c|c|c|c|c|c|c|c|c|c|}
\hline Core & Section & $\begin{array}{l}\text { Depth } \\
\text { (cm) }\end{array}$ & Diff. & $\begin{array}{c}\% \\
\text { Amorphous }\end{array}$ & Calc. & Dolo. & Quar. & $\begin{array}{l}\text { K- } \\
\text { Feld. }\end{array}$ & Plag. & Kaol. & Mica & Chlo. & Mont. & Pyri. & Bari. & Clin. & Phil. \\
\hline 1 & 3 & $6-8$ & 90.1 & 70.9 & 00.0 & 00.0 & 26.0 & 00.0 & 14.6 & 6.2 & 25.0 & 9.6 & 18.5 & 00.0 & 00.0 & 00.0 & 00.0 \\
\hline 1 & 3 & $94-96$ & 90.0 & 70.6 & 00.0 & 00.0 & 32.2 & 00.0 & 17.1 & 10.3 & 28.4 & 11.9 & 00.0 & 00.0 & 00.0 & 00.0 & 00.0 \\
\hline 1 & 4 & $18-19$ & 90.0 & 70.6 & 00.0 & 00.0 & 17.9 & 00.0 & 9.5 & 12.9 & 54.0 & 5.7 & 00.0 & 00.0 & 00.0 & 00.0 & 00.0 \\
\hline 1 & 4 & $80-81$ & 90.0 & 70.6 & 00.0 & 00.0 & 33.4 & 00.0 & 17.7 & 10.7 & 26.8 & 11.4 & 00.0 & 00.0 & 00.0 & 00.0 & 00.0 \\
\hline 1 & 6 & $45-46$ & 90.3 & 71.5 & 00.0 & 00.0 & 31.3 & 00.0 & 16.6 & 13.8 & 30.1 & 8.2 & 00.0 & 00.0 & 00.0 & 00.0 & 00.0 \\
\hline 1 & 6 & $85-86$ & 90.5 & 72.1 & 00.0 & 00.0 & 30.7 & 00.0 & 17.8 & 9.8 & 29.4 & 12.3 & 00.0 & 00.0 & 00.0 & 00.0 & 00.0 \\
\hline 2 & 1 & $16-17$ & 90.0 & 70.6 & 00.0 & 00.0 & 24.8 & 00.0 & 13.4 & 7.9 & 21.8 & 9.9 & 22.1 & 00.0 & 00.0 & 00.0 & 00.0 \\
\hline 2 & 1 & $75-76$ & 90.2 & 71.2 & 00.0 & 00.0 & 26.0 & 00.0 & 13.8 & 9.4 & 21.4 & 10.9 & 18.5 & 00.0 & 00.0 & 00.0 & 00.0 \\
\hline 2 & 2 & $4-5$ & 90.3 & 71.5 & 00.0 & 00.0 & 24.9 & 00.0 & 13.2 & 6.0 & 21.9 & 11.9 & 22.1 & 00.0 & 00.0 & 00.0 & 00.0 \\
\hline 2 & 2 & $74-75$ & 89.8 & 70.0 & 00.0 & 00.0 & 29.2 & 00.0 & 15.8 & 12.9 & 30.4 & 11.7 & 00.0 & 00.0 & 00.0 & 00.0 & 00.0 \\
\hline 2 & 3 & $4-5$ & 89.8 & 70.0 & 00.0 & 00.0 & 32.2 & 00.0 & 17.1 & 10.3 & 28.4 & 11.9 & 00.0 & 00.0 & 00.0 & 00.0 & 00.0 \\
\hline 2 & 3 & $125-126$ & 90.2 & 71.2 & 00.0 & 00.0 & 28.7 & 00.0 & 13.2 & 9.2 & 36.8 & 12.1 & 00.0 & 00.0 & 00.0 & 00.0 & 00.0 \\
\hline 2 & 4 & $16-17$ & 90.0 & 70.6 & 5.4 & 00.0 & 31.8 & 00.0 & 15.3 & 10.2 & 25.5 & 11.8 & 00.0 & 00.0 & 00.0 & 00.0 & 00.0 \\
\hline 2 & 4 & $75-76$ & 90.1 & 70.9 & 00.0 & 00.0 & 36.9 & 00.0 & 16.6 & 7.4 & 23.6 & 15.5 & 00.0 & 00.0 & 00.0 & 00.0 & 00.0 \\
\hline 2 & 5 & $32-34$ & 89.9 & 70.3 & 00.0 & 00.0 & 30.1 & 00.0 & 16.9 & 12.0 & 28.9 & 12.0 & 00.0 & 00.0 & 00.0 & 00.0 & 00.0 \\
\hline 2 & 5 & $103-104$ & 90.0 & 70.6 & 00.0 & 00.0 & 28.1 & 00.0 & 14.0 & 16.8 & 33.7 & 7.3 & 00.0 & 00.0 & 00.0 & 00.0 & 00.0 \\
\hline 2 & 6 & $14-15$ & 90.0 & 70.6 & 00.0 & 00.0 & 31.0 & 00.0 & 16.4 & 9.9 & 29.7 & 13.0 & 00.0 & 00.0 & 00.0 & 00.0 & 00.0 \\
\hline 2 & 6 & $74-75$ & 89.9 & 70.3 & 00.0 & 00.0 & 36.5 & 00.0 & 18.2 & 10.2 & 20.4 & 14.6 & 00.0 & 00.0 & 00.0 & 00.0 & 00.0 \\
\hline 3 & 1 & $24-25$ & 89.9 & 70.3 & 23.4 & 00.0 & 32.5 & 00.0 & 15.6 & 00.0 & 15.6 & 13.0 & 00.0 & 00.0 & 00.0 & 00.0 & 00.0 \\
\hline 3 & 2 & $19-20$ & 90.0 & 70.6 & 00.0 & 00.0 & 36.0 & 00.0 & 19.1 & 8.6 & 23.0 & 13.3 & 00.0 & 00.0 & 00.0 & 00.0 & 00.0 \\
\hline 3 & 2 & $92-93$ & 90.0 & 70.6 & 00.0 & 00.0 & 48.5 & 00.0 & 25.7 & 00.0 & 00.0 & 25.7 & 00.0 & 00.0 & 00.0 & 00.0 & 00.0 \\
\hline 3 & 3 & $126-127$ & 89.6 & 69.4 & 00.0 & 00.0 & 50.5 & 00.0 & 24.2 & 00.0 & 00.0 & 25.3 & 00.0 & 00.0 & 00.0 & 00.0 & 00.0 \\
\hline 3 & 4 & $15-17$ & 90.4 & 71.8 & 7.6 & 00.0 & 33.0 & 00.0 & 16.8 & 9.2 & 23.8 & 9.6 & 00.0 & 00.0 & 00.0 & 00.0 & 00.0 \\
\hline 3 & 4 & $100-101$ & 90.1 & 70.9 & 00.0 & 00.0 & 39.4 & 00.0 & 17.7 & 3.1 & 22.0 & 17.7 & 00.0 & 00.0 & 00.0 & 00.0 & 00.0 \\
\hline 3 & 5 & $10-11$ & 90.3 & 71.5 & 00.0 & 00.0 & 51.3 & 00.0 & 27.2 & 8.2 & 00.0 & 13.3 & 00.0 & 00.0 & 00.0 & 00.0 & 00.0 \\
\hline 3 & 5 & $146-147$ & 89.7 & 69.7 & 32.5 & 00.0 & 26.0 & 00.0 & 13.8 & 5.2 & 12.5 & 10.1 & 00.0 & 00.0 & 00.0 & 00.0 & 00.0 \\
\hline
\end{tabular}


Hole 33 - Continued

\begin{tabular}{|c|c|c|c|c|c|c|c|c|c|c|c|c|c|c|c|c|c|}
\hline Core & Section & $\begin{array}{l}\text { Depth } \\
\text { (cm) }\end{array}$ & Diff. & $\begin{array}{c}\% \\
\text { Amorphous }\end{array}$ & Calc. & Dolo. & Quar. & $\begin{array}{l}\mathrm{K}- \\
\text { Feld. }\end{array}$ & Plag. & Kaol. & Mica & Chlo. & Mont. & Pyri. & Bari. & Clin. & Phil. \\
\hline 3 & 6 & $20-21$ & 90.1 & 70.9 & 00.0 & 00.0 & 37.4 & 00.0 & 19.2 & 12.0 & 21.0 & 9.7 & 00.0 & 00.0 & 00.0 & 00.0 & 00.0 \\
\hline 3 & 6 & 98-99 & 90.2 & 71.2 & 00.0 & 00.0 & 36.1 & 00.0 & 18.4 & 7.2 & 26.0 & 12.3 & 00.0 & 00.0 & 00.0 & 00.0 & 00.0 \\
\hline 4 & 1 & $5-6$ & 89.3 & 68.5 & 34.5 & 00.0 & 26.9 & 00.0 & 13.1 & 2.8 & 13.8 & 9.0 & 00.0 & 00.0 & 00.0 & 00.0 & 00.0 \\
\hline 4 & 1 & $63-64$ & 92.9 & 79.1 & 5.6 & 00.0 & 32.9 & 00.0 & 15.8 & 00.0 & 29.3 & 16.4 & 00.0 & 00.0 & 00.0 & 00.0 & 00.0 \\
\hline 4 & 3 & $38-39$ & 89.9 & 70.3 & 00.0 & 00.0 & 35.1 & 00.0 & 17.5 & 00.0 & 28.8 & 18.6 & 00.0 & 00.0 & 00.0 & 00.0 & 00.0 \\
\hline 4 & 3 & $100-101$ & 89.9 & 70.3 & 6.9 & 00.0 & 36.2 & 00.0 & 18.5 & 00.0 & 23.2 & 15.2 & 00.0 & 00.0 & 00.0 & 00.0 & 00.0 \\
\hline 4 & 5 & 8-9 & 89.9 & 70.3 & 00.0 & 00.0 & 39.7 & 00.0 & 22.2 & 00.0 & 22.2 & 15.9 & 00.0 & 00.0 & 00.0 & 00.0 & 00.0 \\
\hline 4 & 5 & $125-126$ & 90.0 & 70.6 & 23.3 & 00.0 & 30.2 & 00.0 & 16.0 & 00.0 & 16.9 & 13.6 & 00.0 & 00.0 & 00.0 & 00.0 & 00.0 \\
\hline 5 & 3 & $7-8$ & 89.8 & 70.0 & 41.2 & 00.0 & 22.6 & 00.0 & 11.5 & 00.0 & 14.5 & 10.2 & 00.0 & 00.0 & 00.0 & 00.0 & 00.0 \\
\hline 5 & 3 & $120-121$ & 90.5 & 72.1 & 8.6 & 00.0 & 37.2 & 00.0 & 18.6 & 00.0 & 20.8 & 14.9 & 00.0 & 00.0 & 00.0 & 00.0 & 00.0 \\
\hline 5 & 6 & $28-29$ & 90.7 & 72.6 & 22.1 & 00.0 & 46.1 & 00.0 & 20.7 & 00.0 & 00.0 & 11.0 & 00.0 & 00.0 & 00.0 & 00.0 & 00.0 \\
\hline 5 & 6 & $68-69$ & 90.1 & 70.9 & 34.5 & 00.0 & 25.8 & 00.0 & 13.6 & 00.0 & 16.5 & 9.5 & 00.0 & 00.0 & 00.0 & 00.0 & 00.0 \\
\hline 6 & 6 & NDG a & 89.8 & 70.0 & 75.6 & 00.0 & 12.2 & 00.0 & 7.1 & 00.0 & 00.0 & 5.1 & 00.0 & 00.0 & 00.0 & 00.0 & 00.0 \\
\hline 6 & 6 & $134-135$ & 88.6 & 66.5 & 82.4 & 00.0 & 8.6 & 00.0 & 4.3 & 00.0 & 00.0 & 4.7 & 00.0 & 00.0 & 00.0 & 00.0 & 00.0 \\
\hline 7 & 3 & $12-13$ & 91.3 & 74.4 & 21.1 & 00.0 & 14.7 & 00.0 & 13.2 & 00.0 & 00.0 & 11.7 & 39.2 & 00.0 & 00.0 & 00.0 & 00.0 \\
\hline 7 & 3 & $27-28$ & 91.0 & 73.5 & 64.0 & 00.0 & 18.3 & 00.0 & 17.7 & 00.0 & 00.0 & 00.0 & 00.0 & 00.0 & 00.0 & 00.0 & 00.0 \\
\hline 7 & 3 & $108-109$ & 91.0 & 73.5 & 65.1 & 00.0 & 16.9 & 00.0 & 18.0 & 00.0 & 00.0 & 00.0 & 00.0 & 00.0 & 00.0 & 00.0 & 00.0 \\
\hline 8 & 1 & $19-20$ & 90.1 & 70.9 & 51.2 & 00.0 & 22.7 & 00.0 & 12.0 & 00.0 & 00.0 & 14.1 & 00.0 & 00.0 & 00.0 & 00.0 & 00.0 \\
\hline 8 & 1 & $106-107$ & 91.2 & 74.1 & 42.8 & 00.0 & 27.8 & 00.0 & 14.7 & 00.0 & 00.0 & 14.7 & 00.0 & 00.0 & 00.0 & 00.0 & 00.0 \\
\hline 8 & 2 & $25-26$ & 90.8 & 72.9 & 45.1 & 00.0 & 20.0 & 00.0 & 13.1 & 00.0 & 00.0 & 21.8 & 00.0 & 00.0 & 00.0 & 00.0 & 00.0 \\
\hline 8 & 2 & $75-76$ & 91.2 & 74.1 & 00.0 & 00.0 & 50.0 & 00.0 & 30.0 & 00.0 & 00.0 & 20.0 & 00.0 & 00.0 & 00.0 & 00.0 & 00.0 \\
\hline 8 & 3 & $6-7$ & 91.3 & 74.4 & 00.0 & 00.0 & 65.4 & 00.0 & 34.6 & 00.0 & 00.0 & 00.0 & 00.0 & 00.0 & 00.0 & 00.0 & 00.0 \\
\hline 8 & 3 & $75-76$ & 91.3 & 74.4 & 7.7 & 00.0 & 20.4 & 00.0 & 10.8 & 00.0 & 16.7 & 8.1 & 36.2 & 00.0 & 00.0 & 00.0 & 00.0 \\
\hline 8 & 4 & $15-16$ & 91.1 & 73.8 & 14.4 & 00.0 & 37.9 & 00.0 & 22.7 & 00.0 & 00.0 & 25.0 & 00.0 & 00.0 & 00.0 & 00.0 & 00.0 \\
\hline 8 & 4 & $124-125$ & 90.9 & 73.2 & 37.5 & 00.0 & 26.0 & 00.0 & 15.6 & 00.0 & 00.0 & 20.8 & 00.0 & 00.0 & 00.0 & 00.0 & 00.0 \\
\hline 8 & 5 & $46-47$ & 91.2 & 74.1 & 40.4 & 00.0 & 24.8 & 00.0 & 14.9 & 00.0 & 00.0 & 19.8 & 00.0 & 00.0 & 00.0 & 00.0 & 00.0 \\
\hline
\end{tabular}


Results of X-Ray Diffraction Analysis of Bulk Samples from Leg 5 - Continued

Hole 33 - Continued

\begin{tabular}{|c|c|c|c|c|c|c|c|c|c|c|c|c|c|c|c|c|c|}
\hline Core & Section & $\begin{array}{l}\text { Depth } \\
(\mathrm{cm})\end{array}$ & Diff. & $\begin{array}{c}\% \\
\text { Amorphous }\end{array}$ & Calc. & Dolo. & Quar. & $\begin{array}{l}\text { K- } \\
\text { Feld. }\end{array}$ & Plag. & Kaol. & Mica & Chlo. & Mont. & Pyri. & Bari. & Clin. & Phil. \\
\hline 8 & 5 & $111-112$ & 90.9 & 73.2 & 17.5 & 00.0 & 56.6 & 00.0 & 26.0 & 00.0 & 00.0 & 00.0 & 00.0 & 00.0 & 00.0 & 00.0 & 00.0 \\
\hline 8 & 6 & $14-15$ & 91.1 & 73.8 & 26.0 & 00.0 & 33.8 & 00.0 & 17.7 & 00.0 & 00.0 & 22.3 & 00.0 & 00.0 & 00.0 & 00.0 & 00.0 \\
\hline 8 & 6 & $93-94$ & 90.8 & 72.9 & 29.9 & 00.0 & 41.8 & 00.0 & 28.3 & 00.0 & 00.0 & 00.0 & 00.0 & 00.0 & 00.0 & 00.0 & 00.0 \\
\hline 9 & 3 & $16-17$ & 91.2 & 74.1 & 00.0 & 00.0 & 37.6 & 00.0 & 22.6 & 15.0 & 00.0 & 24.8 & 00.0 & 00.0 & 00.0 & 00.0 & 00.0 \\
\hline 9 & 3 & $123-124$ & 90.7 & 72.6 & 00.0 & 00.0 & 51.4 & 00.0 & 27.5 & 00.0 & 00.0 & 21.2 & 00.0 & 00.0 & 00.0 & 00.0 & 00.0 \\
\hline 9 & 6 & $10-11$ & 90.6 & 72.4 & 00.0 & 00.0 & 49.2 & 00.0 & 23.6 & 00.0 & 00.0 & 27.2 & 00.0 & 00.0 & 00.0 & 00.0 & 00.0 \\
\hline 9 & 6 & $134-135$ & 90.8 & 72.9 & 23.0 & 00.0 & 23.9 & 00.0 & 14.4 & 00.0 & 19.6 & 19.1 & 00.0 & 00.0 & 00.0 & 00.0 & 00.0 \\
\hline 10 & 2 & $5-6$ & 90.1 & 70.9 & 85.7 & 00.0 & 10.5 & 00.0 & 3.9 & 00.0 & 00.0 & 00.0 & 00.0 & 00.0 & 00.0 & 00.0 & 00.0 \\
\hline 10 & 5 & $13-14$ & 90.9 & 73.2 & 14.9 & 00.0 & 37.3 & 00.0 & 21.3 & 00.0 & 00.0 & 26.6 & 00.0 & 00.0 & 00.0 & 00.0 & 00.0 \\
\hline 10 & 5 & $75-76$ & 90.4 & 71.8 & 39.4 & 00.0 & 29.4 & 00.0 & 15.6 & 00.0 & 00.0 & 15.6 & 00.0 & 00.0 & 00.0 & 00.0 & 00.0 \\
\hline 11 & 2 & $31-33$ & 88.5 & 66.2 & 92.6 & 00.0 & 4.5 & 00.0 & 2.9 & 00.0 & 00.0 & 00.0 & 00.0 & 00.0 & 00.0 & 00.0 & 00.0 \\
\hline 11 & 2 & $131-132$ & 88.9 & 67.4 & 90.7 & 00.0 & 5.9 & 00.0 & 3.4 & 00.0 & 00.0 & 00.0 & 00.0 & 00.0 & 00.0 & 00.0 & 00.0 \\
\hline 11 & 3 & $10-11$ & 90.7 & 72.6 & 00.0 & 00.0 & 14.0 & 00.0 & 12.6 & 5.6 & 5.6 & 00.0 & 62.2 & 00.0 & 00.0 & 00.0 & 00.0 \\
\hline 11 & 3 & $73-74$ & 70.7 & 72.6 & 00.0 & 00.0 & 12.3 & 00.0 & 9.0 & 5.9 & 6.9 & 00.0 & 65.8 & 00.0 & 00.0 & 00.0 & 00.0 \\
\hline 12 & 3 & $9-10$ & 91.0 & 73.5 & 00.0 & 00.0 & 16.0 & 00.0 & 14.4 & 12.8 & 00.0 & 00.0 & 56.9 & 00.0 & 00.0 & 00.0 & 00.0 \\
\hline 12 & 6 & $75-76$ & 90.7 & 72.6 & 00.0 & 00.0 & 12.5 & 00.0 & 9.4 & 00.0 & 00.0 & 00.0 & 78.1 & 00.0 & 00.0 & 00.0 & 00.0 \\
\hline 15 & 1 & $29-30$ & 91.0 & 73.5 & 00.0 & 00.0 & 70.9 & 00.0 & 29.1 & 00.0 & 00.0 & 00.0 & 00.0 & 00.0 & 00.0 & 00.0 & 00.0 \\
\hline 15 & 1 & $100-102$ & 87.9 & 64.4 & 94.1 & 00.0 & 4.4 & 00.0 & 1.5 & 00.0 & 00.0 & 00.0 & 00.0 & 00.0 & 00.0 & 00.0 & 00.0 \\
\hline \multicolumn{18}{|c|}{ Hole 34} \\
\hline Core & Section & $\begin{array}{l}\text { Depth } \\
(\mathrm{cm})\end{array}$ & Diff. & $\begin{array}{c}\% \\
\text { Amorphous }\end{array}$ & Calc. & Dolo. & Quar. & $\begin{array}{l}\text { K- } \\
\text { Feld. }\end{array}$ & Plag. & Kaol. & Mica & Chlo. & Mont. & Pyri. & Bari. & Clin. & Phil. \\
\hline 1 & 1 & $11-12$ & 89.3 & 68.5 & 00.0 & 00.0 & 37.4 & 00.0 & 19.1 & 4.5 & 24.0 & 15.0 & 00.0 & 00.0 & 00.0 & 00.0 & 00.0 \\
\hline 1 & 1 & $130-131$ & 89.1 & 67.9 & 00.0 & 00.0 & 33.3 & 00.0 & 19.7 & 8.0 & 26.7 & 12.3 & 00.0 & 00.0 & 00.0 & 00.0 & 00.0 \\
\hline 1 & 2 & $7-8$ & 89.4 & 68.8 & 00.0 & 00.0 & 31.6 & 00.0 & 16.8 & 12.6 & 30.4 & 8.5 & 00.0 & 00.0 & 00.0 & 00.0 & 00.0 \\
\hline
\end{tabular}


Results of X-Ray Diffraction Analysis of Bulk Samples from Leg 5 - Continued

Hole 34 - Continued

\begin{tabular}{|c|c|c|c|c|c|c|c|c|c|c|c|c|c|c|c|c|c|}
\hline Core & Section & $\begin{array}{l}\text { Depth } \\
\text { (cm) }\end{array}$ & Diff. & $\begin{array}{c}\% \\
\text { Amorphous }\end{array}$ & Calc. & Dolo. & Quar. & $\begin{array}{l}\text { K- } \\
\text { Feld. }\end{array}$ & Plag. & Kaol. & Mica & Chlo. & Mont. & Pyri. & Bari. & Clin. & Phil. \\
\hline 1 & 2 & 134-135 & 89.2 & 68.2 & 00.0 & 00.0 & 33.1 & 00.0 & 18.5 & 7.9 & 29.1 & 11.2 & 00.0 & 00.0 & 00.0 & 00.0 & 00.0 \\
\hline 1 & 3 & $14-15$ & 89.3 & 68.5 & 19.6 & 00.0 & 25.8 & 00.0 & 14.5 & 8.3 & 24.8 & 7.0 & 00.0 & 00.0 & 00.0 & 00.0 & 00.0 \\
\hline 1 & 3 & $116-117$ & 89.8 & 70.0 & 00.0 & 00.0 & 36.9 & 00.0 & 22.1 & 4.4 & 20.7 & 15.9 & 00.0 & 00.0 & 00.0 & 00.0 & 00.0 \\
\hline 1 & 4 & $45-46$ & 89.4 & 68.8 & 00.0 & 00.0 & 34.7 & 00.0 & 17.7 & 12.5 & 25.0 & 10.1 & 00.0 & 00.0 & 00.0 & 00.0 & 00.0 \\
\hline 1 & 4 & $133-134$ & 89.4 & 68.8 & 00.0 & 00.0 & 36.9 & 00.0 & 19.9 & 5.9 & 23.6 & 13.6 & 00.0 & 00.0 & 00.0 & 00.0 & 00.0 \\
\hline 1 & 5 & $27-28$ & 89.5 & 69.1 & 00.0 & 00.0 & 33.1 & 00.0 & 18.5 & 10.6 & 27.2 & 10.6 & 00.0 & 00.0 & 00.0 & 00.0 & 00.0 \\
\hline 1 & 5 & $125-126$ & 89.3 & 68.5 & 00.0 & 00.0 & 35.3 & 00.0 & 17.0 & 5.6 & 29.0 & 13.1 & 00.0 & 00.0 & 00.0 & 00.0 & 00.0 \\
\hline 1 & 6 & $17-18$ & 89.4 & 68.8 & 00.0 & 00.0 & 36.4 & 00.0 & 18.2 & 14.5 & 23.3 & 7.6 & 00.0 & 00.0 & 00.0 & 00.0 & 00.0 \\
\hline 1 & 6 & $125-126$ & 89.3 & 68.5 & 00.0 & 00.0 & 39.1 & 00.0 & 19.5 & 00.0 & 25.0 & 16.4 & 00.0 & 00.0 & 00.0 & 00.0 & 00.0 \\
\hline 2 & 1 & $15-16$ & 89.6 & 69.4 & 00.0 & 00.0 & 36.5 & 00.0 & 19.7 & 8.8 & 26.3 & 8.8 & 00.0 & 00.0 & 00.0 & 00.0 & 00.0 \\
\hline 2 & 1 & $74-75$ & 89.6 & 69.4 & 00.0 & 00.0 & 33.4 & 00.0 & 18.1 & 9.4 & 30.1 & 9.0 & 00.0 & 00.0 & 00.0 & 00.0 & 00.0 \\
\hline 2 & 2 & $12-13$ & 89.0 & 67.6 & 00.0 & 00.0 & 42.4 & 00.0 & 24.2 & 5.1 & 20.3 & 8.0 & 00.0 & 00.0 & 00.0 & 00.0 & 00.0 \\
\hline 2 & 2 & $74-76$ & 89.1 & 67.9 & 00.0 & 00.0 & 37.0 & 00.0 & 21.8 & 10.4 & 20.7 & 10.0 & 00.0 & 00.0 & 00.0 & 00.0 & 00.0 \\
\hline 2 & 3 & $11-12$ & 89.6 & 69.4 & 00.0 & 00.0 & 43.8 & 00.0 & 22.4 & 00.0 & 17.5 & 16.2 & 00.0 & 00.0 & 00.0 & 00.0 & 00.0 \\
\hline 2 & 3 & $74-75$ & 89.8 & 70.0 & 00.0 & 00.0 & 33.4 & 00.0 & 17.7 & 21.4 & 27.4 & 00.0 & 00.0 & 00.0 & 00.0 & 00.0 & 00.0 \\
\hline 2 & 4 & $11-12$ & 89.4 & 68.8 & 00.0 & 00.0 & 40.3 & 00.0 & 21.4 & 4.8 & 22.6 & 10.9 & 00.0 & 00.0 & 00.0 & 00.0 & 00.0 \\
\hline 2 & 4 & $74-75$ & 89.7 & 69.7 & 00.0 & 00.0 & 35.0 & 00.0 & 19.2 & 16.8 & 22.4 & 6.6 & 00.0 & 00.0 & 00.0 & 00.0 & 00.0 \\
\hline 2 & 5 & $8-9$ & 89.6 & 69.4 & 00.0 & 00.0 & 37.6 & 00.0 & 19.2 & 15.0 & 21.0 & 7.1 & 00.0 & 00.0 & 00.0 & 00.0 & 00.0 \\
\hline 2 & 5 & $78-79$ & 88.4 & 65.9 & 46.8 & 00.0 & 13.9 & 00.0 & 7.3 & 2.9 & 7.8 & 3.9 & 17.3 & 00.0 & 00.0 & 00.0 & 00.0 \\
\hline 2 & 6 & $8-9$ & 89.4 & 68.8 & 00.0 & 00.0 & 35.7 & 00.0 & 18.9 & 10.0 & 25.7 & 9.6 & 00.0 & 00.0 & 00.0 & 00.0 & 00.0 \\
\hline 2 & 6 & $75-76$ & 88.7 & 66.8 & 50.8 & 00.0 & 19.3 & 00.0 & 9.5 & 2.1 & 12.7 & 5.6 & 00.0 & 00.0 & 00.0 & 00.0 & 00.0 \\
\hline 3 & 1 & $10-11$ & 87.7 & 63.8 & 63.4 & 00.0 & 8.9 & 00.0 & 4.3 & 00.0 & 7.9 & 3.6 & 11.9 & 00.0 & 00.0 & 00.0 & 00.0 \\
\hline 3 & 1 & $74-75$ & 88.7 & 66.8 & 37.4 & 00.0 & 25.2 & 00.0 & 12.6 & 00.0 & 14.1 & 10.6 & 00.0 & 00.0 & 00.0 & 00.0 & 00.0 \\
\hline 3 & 2 & 8-9 & 88.2 & 65.3 & 59.6 & 00.0 & 14.9 & 00.0 & 7.1 & 00.0 & 12.4 & 5.9 & 00.0 & 00.0 & 00.0 & 00.0 & 00.0 \\
\hline 3 & 2 & $71-72$ & 87.5 & 63.2 & 78.7 & 00.0 & 11.1 & 00.0 & 4.9 & 00.0 & 00.0 & 5.3 & 00.0 & 00.0 & 00.0 & 00.0 & 00.0 \\
\hline 3 & 3 & $10-12$ & 87.9 & 64.4 & 62.9 & 00.0 & 18.9 & 00.0 & 8.2 & 00.0 & 00.0 & 10.1 & 00.0 & 00.0 & 00.0 & 00.0 & 00.0 \\
\hline 3 & 3 & $71-72$ & 88.3 & 65.6 & 64.9 & 00.0 & 17.8 & 00.0 & 9.1 & 00.0 & 00.0 & 8.1 & 00.0 & 00.0 & 00.0 & 00.0 & 00.0 \\
\hline
\end{tabular}


Results of X-Ray Diffraction Analysis of Bulk Samples from Leg 5 - Continued

Hole $34-$ Continued

\begin{tabular}{|c|c|c|c|c|c|c|c|c|c|c|c|c|c|c|c|c|c|}
\hline Core & Section & $\begin{array}{l}\text { Depth } \\
(\mathrm{cm})\end{array}$ & Diff. & $\begin{array}{c}\% \\
\text { Amorphous }\end{array}$ & Calc. & Dolo. & Quar. & $\begin{array}{l}\text { K- } \\
\text { Feld. }\end{array}$ & Plag. & Kaol. & Mica & Chlo. & Mont. & Pyri. & Bari. & Clin. & Phil. \\
\hline 3 & 4 & $11-12$ & 88.6 & 66.5 & 50.5 & 00.0 & 19.5 & 00.0 & 9.5 & 00.0 & 10.5 & 10.0 & 00.0 & 00.0 & 00.0 & 00.0 & 00.0 \\
\hline 3 & 4 & $71-72$ & 87.8 & 64.1 & 70.3 & 00.0 & 11.0 & 00.0 & 5.9 & 2.9 & 5.9 & 4.0 & 00.0 & 00.0 & 00.0 & 00.0 & 00.0 \\
\hline 3 & 5 & $7-8$ & 87.9 & 64.4 & 66.2 & 00.0 & 12.8 & 00.0 & 7.2 & 00.0 & 8.3 & 5.5 & 00.0 & 00.0 & 00.0 & 00.0 & 00.0 \\
\hline 3 & 5 & $74-75$ & 87.9 & 64.4 & 65.5 & 00.0 & 14.0 & 00.0 & 6.8 & 00.0 & 8.2 & 5.5 & 00.0 & 00.0 & 00.0 & 00.0 & 00.0 \\
\hline 3 & 6 & $10-11$ & 88.2 & 65.3 & 59.1 & 00.0 & 15.4 & 00.0 & 8.3 & 00.0 & 9.8 & 7.4 & 00.0 & 00.0 & 00.0 & 00.0 & 00.0 \\
\hline 3 & 6 & $75-76$ & 87.7 & 63.8 & 65.1 & 00.0 & 18.0 & 00.0 & 6.1 & 00.0 & 5.4 & 5.4 & 00.0 & 00.0 & 00.0 & 00.0 & 00.0 \\
\hline 4 & 1 & $16-17$ & 89.7 & 69.7 & 00.0 & 00.0 & 45.2 & 00.0 & 22.6 & 5.4 & 18.1 & 8.6 & 00.0 & 00.0 & 00.0 & 00.0 & 00.0 \\
\hline 4 & 1 & $71-72$ & 89.7 & 69.7 & 00.0 & 00.0 & 36.8 & 00.0 & 17.3 & 2.9 & 14.7 & 8.8 & 19.5 & 00.0 & 00.0 & 00.0 & 00.0 \\
\hline 4 & 2 & $8-9$ & 89.7 & 69.7 & 00.0 & 00.0 & 45.0 & 00.0 & 21.2 & 3.6 & 18.0 & 12.2 & 00.0 & 00.0 & 00.0 & 00.0 & 00.0 \\
\hline 4 & 2 & $70-71$ & 89.8 & 70.0 & 00.0 & 00.0 & 52.6 & 00.0 & 26.8 & 10.5 & 00.0 & 10.0 & 00.0 & 00.0 & 00.0 & 00.0 & 00.0 \\
\hline 4 & 3 & $28-29$ & 89.6 & 69.4 & 00.0 & 00.0 & 41.8 & 00.0 & 20.1 & 23.8 & 00.0 & 14.2 & 00.0 & 00.0 & 00.0 & 00.0 & 00.0 \\
\hline 4 & 3 & $99-100$ & 89.6 & 69.4 & 00.0 & 00.0 & 33.1 & 00.0 & 16.2 & 2.6 & 21.8 & 8.6 & 17.5 & 00.0 & 00.0 & 00.0 & 00.0 \\
\hline 4 & 4 & $14-15$ & 89.5 & 69.1 & 00.0 & 00,0 & 31.2 & 00.0 & 15.3 & 2.5 & 15.3 & 8.1 & 27.7 & 00.0 & 00.0 & 00.0 & 00.0 \\
\hline 4 & 4 & $131-132$ & 89.6 & 69.4 & 00.0 & 00.0 & 32.6 & 00.0 & 16.6 & 6.5 & 13.4 & 7.8 & 23.1 & 00.0 & 00.0 & 00.0 & 00.0 \\
\hline 4 & 5 & $6-7$ & 89.5 & 69.1 & 00.0 & 00.0 & 28.3 & 00.0 & 15.0 & 5.7 & 13.9 & 3.7 & 30.3 & 3.1 & 00.0 & 00.0 & 00.0 \\
\hline 4 & 5 & $125-126$ & 89.3 & 68.5 & 17.7 & 00.0 & 24.3 & 00.0 & 11.9 & 4.8 & 13.8 & 5.8 & 21.6 & 00.0 & 00.0 & 00.0 & 00.0 \\
\hline 4 & 6 & 10-11 & 89.4 & 68.8 & 00.0 & 00.0 & 29.2 & 00.0 & 14.0 & 00.0 & 16.7 & 10.8 & 26.7 & 3.2 & 00.0 & 00.0 & 00.0 \\
\hline 4 & 6 & $124-125$ & 89.7 & 69.7 & 8.5 & 00.0 & 24.4 & 00.0 & 12.9 & 00.0 & 12.0 & 9.0 & 30.5 & 2.7 & 00.0 & 00.0 & 00.0 \\
\hline 5 & 1 & $3-4$ & 90.5 & 72.1 & 00.0 & 00.0 & 40.0 & 00.0 & 21.2 & 00.0 & 19.6 & 14.8 & 00.0 & 4.4 & 00.0 & 00.0 & 00.0 \\
\hline 5 & 1 & $139-140$ & 89.7 & 69.7 & 00.0 & 00.0 & 42.6 & 00.0 & 23.0 & 00.0 & 17.4 & 17.0 & 00.0 & 00.0 & 00.0 & 00.0 & 00.0 \\
\hline 5 & 2 & $30-31$ & 89.5 & 69.1 & 00.0 & 00.0 & 30.3 & 00.0 & 16.7 & 00.0 & 17.3 & 8.8 & 27.0 & 00.0 & 00.0 & 00.0 & 00.0 \\
\hline 5 & 2 & $123-124$ & 90.2 & 71.2 & 00.0 & 00.0 & 41.7 & 00.0 & 21.2 & 00.0 & 17.1 & 15.4 & 00.0 & 4.6 & 00.0 & 00.0 & 00.0 \\
\hline 5 & 3 & $42-43$ & 89.6 & 69.4 & 00.0 & 00.0 & 32.6 & 00.0 & 17.3 & 00.0 & 10.7 & 10.4 & 29.0 & 00.0 & 00.0 & 00.0 & 00.0 \\
\hline 5 & 4 & $87-88$ & 89.5 & 69.1 & 00.0 & 00.0 & 38.6 & 00.0 & 20.8 & 00.0 & 22.0 & 14.3 & 00.0 & 4.2 & 00.0 & 00.0 & 00.0 \\
\hline 5 & 5 & $75-78$ & 89.5 & 69.1 & 00.0 & 00.0 & 41.2 & 00.0 & 23.4 & 00.0 & 16.9 & 14.0 & 00.0 & 4.5 & 00.0 & 00.0 & 00.0 \\
\hline 5 & 6 & $15-16$ & 89.9 & 70.3 & 00.0 & 00.0 & 31.0 & 00.0 & 15.2 & 00.0 & 17.6 & 11.4 & 22.0 & 2.8 & 00.0 & 00.0 & 00.0 \\
\hline 5 & 6 & $62-63$ & 90.4 & 71.8 & 00.0 & 00.0 & 50.2 & 00.0 & 28.6 & 00.0 & 00.0 & 21.1 & 00.0 & 00.0 & 00.0 & 00.0 & 00.0 \\
\hline
\end{tabular}


Hole 34 -Continued

\begin{tabular}{|c|c|c|c|c|c|c|c|c|c|c|c|c|c|c|c|c|c|}
\hline Core & Section & $\begin{array}{l}\text { Depth } \\
\text { (cm) }\end{array}$ & Diff. & $\begin{array}{c}\% \\
\text { Amorphous }\end{array}$ & Calc. & Dolo. & Quar. & $\begin{array}{l}\text { K- } \\
\text { Feld. }\end{array}$ & Plag. & Kaol. & Mica & Chlo. & Mont. & Pyri. & Bari. & Clin. & Phil. \\
\hline 6 & 1 & $36-37$ & 88.1 & 65.0 & 00.0 & 00.0 & 55.6 & 00.0 & 33.3 & 00.0 & 00.0 & 7.2 & 00.0 & 3.9 & 00.0 & 00.0 & 00.0 \\
\hline 6 & 1 & $42-43$ & 87.3 & 62.6 & 00.0 & 00.0 & 44.8 & 00.0 & 31.8 & 00.0 & 00.0 & 4.9 & 16.1 & 2.2 & 00.0 & 00.0 & 00.0 \\
\hline 6 & 2 & $30-31$ & 87.6 & 63.5 & 00.0 & 00.0 & 48.3 & 00.0 & 25.6 & 00.0 & 00.0 & 5.3 & 17.4 & 3.4 & 00.0 & 00.0 & 00.0 \\
\hline 6 & 2 & $50-51$ & 90.0 & 70.6 & 00.0 & 00.0 & 33.2 & 00.0 & 18.3 & 4.0 & 11.0 & 6.3 & 23.6 & 4.6 & 00.0 & 00.0 & 00.0 \\
\hline 6 & 3 & $85-86$ & 89.1 & 67.9 & 13.6 & 00.0 & 38.8 & 00.0 & 19.0 & 00.0 & 12.8 & 12.4 & 00.0 & 3.4 & 00.0 & 00.0 & 00.0 \\
\hline 6 & 4 & $60-61$ & 88.9 & 67.4 & 00.0 & 00.0 & 54.0 & 00.0 & 29.7 & 4.3 & 00.0 & 7.0 & 00.0 & 4.9 & 00.0 & 00.0 & 00.0 \\
\hline 6 & 4 & $137-138$ & 88.8 & 67.1 & 00.0 & 00.0 & 54.3 & 00.0 & 32.1 & 00.0 & 00.0 & 8.7 & 00.0 & 4.9 & 00.0 & 00.0 & 00.0 \\
\hline 6 & 5 & $94-95$ & 89.8 & 70.0 & 00.0 & 00.0 & 45.1 & 00.0 & 23.7 & 5.2 & 17.7 & 10.3 & 00.0 & 00.0 & 00.0 & 00.0 & 00.0 \\
\hline 6 & 5 & $144-145$ & 89.2 & 68.2 & 00.0 & 00.0 & 34.5 & 00.0 & 19.0 & 4.1 & 14.1 & 3.8 & 24.5 & 00.0 & 00.0 & 00.0 & 00.0 \\
\hline 6 & 6 & $28-29$ & 89.3 & 68.5 & 00.0 & 00.0 & 30.8 & 00.0 & 16.6 & 00.0 & 15.1 & 7.4 & 27.4 & 2.7 & 00.0 & 00.0 & 00.0 \\
\hline 6 & 6 & $134-135$ & 90.2 & 71.2 & 11.5 & 00.0 & 42.7 & 00.0 & 23.1 & 00.0 & 00.0 & 17.9 & 00.0 & 4.7 & 00.0 & 00.0 & 00.0 \\
\hline 7 & 3 & $55-56$ & 90.0 & 70.6 & 00.0 & 00.0 & 42.5 & 00.0 & 20.8 & 00.0 & 17.4 & 19.1 & 00.0 & 00.0 & 00.0 & 00.0 & 00.0 \\
\hline 7 & 3 & $120-121$ & 89.9 & 70.3 & 23.5 & 00.0 & 21.7 & 00.0 & 10.6 & 00.0 & 14.3 & 8.0 & 19.3 & 2.4 & 00.0 & 00.0 & 00.0 \\
\hline 7 & 4 & $32-33$ & 89.6 & 69.4 & 20.5 & 00.0 & 33.5 & 00.0 & 16.1 & 00.0 & 16.4 & 13.4 & 00.0 & 00.0 & 00.0 & 00.0 & 00.0 \\
\hline 7 & 4 & $133-134$ & 89.7 & 69.7 & 11.9 & 00.0 & 25.8 & 00.0 & 12.4 & 00.0 & 14.7 & 7.5 & 27.6 & 00.0 & 00.0 & 00.0 & 00.0 \\
\hline 7 & 6 & $12-13$ & 89.5 & 69.1 & 12.9 & 00.0 & 35.8 & 00.0 & 19.3 & 00.0 & 14.7 & 17.2 & 00.0 & 00.0 & 00.0 & 00.0 & 00.0 \\
\hline 7 & 6 & $87-88$ & 90.0 & 70.6 & 27.2 & 00.0 & 31.6 & 00.0 & 15.5 & 00.0 & 15.5 & 10.0 & 00.0 & 00.0 & 00.0 & 00.0 & 00.0 \\
\hline 8 & 1 & $30-31$ & 90.2 & 71.2 & 15.3 & 00.0 & 25.5 & 00.0 & 14.5 & 00.0 & 12.5 & 9.4 & 22.7 & 00.0 & 00.0 & 00.0 & 00.0 \\
\hline 8 & 2 & $17-18$ & 90.0 & 70.6 & 27.7 & 00.0 & 20.1 & 00.0 & 11.8 & 00.0 & 11.4 & 7.4 & 21.5 & 00.0 & 00.0 & 00.0 & 00.0 \\
\hline 8 & 4 & $24-25$ & 89.8 & 70.0 & 20.1 & 00.0 & 25.4 & 00.0 & 13.7 & 00.0 & 12.5 & 10.3 & 18.0 & 00.0 & 00.0 & 00.0 & 00.0 \\
\hline 8 & 5 & $20-21$ & 90.8 & 72.9 & 00.0 & 00.0 & 43.8 & 00.0 & 24.1 & 00.0 & 18.0 & 14.0 & 00.0 & 00.0 & 00.0 & 00.0 & 00.0 \\
\hline 8 & 6 & $9-10$ & 89.6 & 69.4 & 00.0 & 00.0 & 40.5 & 00.0 & 24.7 & 00.0 & 19.8 & 15.0 & 00.0 & 00.0 & 00.0 & 00.0 & 00.0 \\
\hline 9 & 1 & $45-46$ & 90.0 & 70.6 & 35.3 & 00.0 & 35.3 & 00.0 & 19.1 & 00.0 & 00.0 & 10.2 & 00.0 & 00.0 & 00.0 & 00.0 & 00.0 \\
\hline 9 & 1 & $104-105$ & 91.1 & 73.8 & 00.0 & 00.0 & 22.4 & 00.0 & 12.8 & 00.0 & 18.4 & 6.5 & 35.9 & 4.0 & 00.0 & 00.0 & 00.0 \\
\hline 9 & 2 & $131-132$ & 91.2 & 74.1 & 12.1 & 00.0 & 20.9 & 00.0 & 11.1 & 00.0 & 00.0 & 6.0 & 44.7 & 5.2 & 00.0 & 00.0 & 00.0 \\
\hline 9 & 3 & $78-79$ & 90.9 & 73.2 & 00.0 & 00.0 & 23.2 & 00.0 & 13.7 & 8.3 & 11.4 & 2.5 & 37.1 & 3.7 & 00.0 & 00.0 & 00.0 \\
\hline
\end{tabular}


Results of X-Ray Diffraction Analysis of Bulk Samples from Leg 5 - Continued

Hole 34 - Continued

\begin{tabular}{|c|c|c|c|c|c|c|c|c|c|c|c|c|c|c|c|c|c|}
\hline Core & Section & $\begin{array}{l}\text { Depth } \\
\text { (cm) }\end{array}$ & Diff. & $\begin{array}{c}\% \\
\text { Amorphous }\end{array}$ & Calc. & Dolo. & Quar. & $\begin{array}{l}\text { K- } \\
\text { Feld. }\end{array}$ & Plag. & Kaol. & Mica & Chlo. & Mont. & Pyri. & Bari. & Clin. & Phil. \\
\hline 9 & 4 & 67 & 91.0 & 73.5 & 00.0 & 00.0 & 22.6 & 00.0 & 12.4 & 5.4 & 16.3 & 2.9 & 40.3 & 00.0 & 00.0 & 00.0 & 00.0 \\
\hline 9 & 4 & $142-143$ & 90.9 & 73.2 & 00.0 & 00.0 & 16.0 & 00.0 & 9.4 & 6.4 & 14.0 & 00.0 & 51.2 & 2.9 & 00.0 & 00.0 & 00.0 \\
\hline 9 & 5 & $37-38$ & 91.1 & 73.8 & 00.0 & 00.0 & 23.2 & 00.0 & 13.7 & 1.8 & 16.7 & 3.0 & 41.3 & 00.0 & 00.0 & 00.0 & 00.0 \\
\hline 9 & 5 & $124-125$ & 91.0 & 73.5 & 00.0 & 00.0 & 21.5 & 00.0 & 12.7 & 9.5 & 17.7 & 00.0 & 38.4 & 00.0 & 00.0 & 00.0 & 00.0 \\
\hline 9 & 6 & $25-26$ & 90.9 & 73.2 & 00.0 & 00.0 & 19.3 & 00.0 & 10.6 & 3.8 & 10.8 & 3.6 & 48.1 & 3.4 & 00.0 & 00.0 & 00.0 \\
\hline 9 & 6 & $125-126$ & 90.8 & 72.9 & 00.0 & 00.0 & 18.8 & 00.0 & 10.3 & 00.0 & 13.5 & 3.9 & 50.2 & 3.0 & 00.0 & 00.0 & 00.0 \\
\hline 10 & 1 & $107-108$ & 91.5 & 75.0 & 00.0 & 00.0 & 37.7 & 00.0 & 19.6 & 00.0 & 21.1 & 9.8 & 00.0 & 11.3 & 00.0 & 00.0 & 00.0 \\
\hline 10 & 1 & $113-114$ & 91.0 & 73.5 & 16.2 & 00.0 & 21.4 & 00.0 & 15.2 & 00.0 & 12.0 & 8.5 & 26.5 & 00.0 & 00.0 & 00.0 & 00.0 \\
\hline 10 & 2 & $12-13$ & 90.5 & 72.1 & 51.8 & 00.0 & 21.6 & 00.0 & 14.3 & 00.0 & 00.0 & 12.1 & 00.0 & 00.0 & 00.0 & 00.0 & 00.0 \\
\hline 10 & 2 & $74-76$ & 91.1 & 73.8 & 22.6 & 00.0 & 19.8 & 00.0 & 14.1 & 00.0 & 00.0 & 7.9 & 35.3 & 00.0 & 00.0 & 00.0 & 00.0 \\
\hline 10 & 3 & $10-11$ & 91.2 & 74.1 & 00.0 & 00.0 & 46.2 & 00.0 & 34.7 & 12.9 & 00.0 & 6.0 & 00.0 & 00.0 & 00.0 & 00.0 & 00.0 \\
\hline 10 & 3 & $72-73$ & 91.3 & 74.4 & 9.5 & 00.0 & 14.1 & 00.0 & 8.5 & 5.6 & 11.6 & 00.0 & 50.4 & 00.0 & 00.0 & 00.0 & 00.0 \\
\hline 10 & 4 & $13-14$ & 91.2 & 74.1 & 00.0 & 00.0 & 19.4 & 00.0 & 13.8 & 9.3 & 15.9 & 00.0 & 41.4 & 00.0 & 00.0 & 00.0 & 00.0 \\
\hline 10 & 4 & $128-129$ & 91.2 & 74.1 & 11.0 & 00.0 & 19.1 & 00.0 & 12.6 & 00.0 & 15.6 & 7.6 & 33.9 & 00.0 & 00.0 & 00.0 & 00.0 \\
\hline 10 & 5 & $42-43$ & 90.6 & 72.4 & 35.4 & 00.0 & 12.3 & 00.0 & 7.2 & 00.0 & 11.8 & 7.0 & 26.2 & 00.0 & 00.0 & 00.0 & 00.0 \\
\hline 10 & 5 & $126-127$ & 90.7 & 72.6 & 50.1 & 00.0 & 10.4 & 00.0 & 7.8 & 00.0 & 12.5 & 5.2 & 13.8 & 00.0 & 00.0 & 00.0 & 00.0 \\
\hline 10 & 6 & $43-44$ & 90.7 & 72.6 & 46.4 & 00.0 & 18.4 & 00.0 & 12.1 & 4.8 & 13.5 & 4.6 & 00.0 & 00.0 & 00.0 & 00.0 & 00.0 \\
\hline 10 & 6 & $135-136$ & 90.8 & 72.9 & 27.9 & 00.0 & 11.4 & 00.0 & 11.3 & 00.0 & 10.4 & 7.7 & 30.9 & 00.0 & 00.0 & 00.0 & 00.0 \\
\hline 11 & 2 & $17-18$ & 91.0 & 73.5 & 00.0 & 00.0 & 33.1 & 00.0 & 24.8 & 00.0 & 29.1 & 12.9 & 00.0 & 00.0 & 00.0 & 00.0 & 00.0 \\
\hline 11 & 2 & $71-72$ & 90.5 & 72.1 & 28.3 & 00.0 & 12.5 & 00.0 & 11.0 & 00.0 & 12.0 & 9.7 & 26.2 & 00.0 & 00.0 & 00.0 & 00.0 \\
\hline 11 & 3 & $26-27$ & 91.0 & 73.5 & 18.6 & 00.0 & 12.2 & 00.0 & 9.1 & 00.0 & 11.9 & 4.7 & 43.3 & 00.0 & 00.0 & 00.0 & 00.0 \\
\hline 11 & 3 & $116-117$ & 90.9 & 73.2 & 8.7 & 00.0 & 18.2 & 00.0 & 13.6 & 00.0 & 14.9 & 12.0 & 32.4 & 00.0 & 00.0 & 00.0 & 00.0 \\
\hline 12 & 1 & $92-93$ & 91.4 & 74.7 & 00.0 & 00.0 & 16.1 & 00.0 & 15.6 & 00.0 & 00.0 & 10.6 & 57.5 & 00.0 & 00.0 & 00.0 & 00.0 \\
\hline 12 & 1 & $135-136$ & 90.9 & 73.2 & 00.0 & 00.0 & 19.9 & 00.0 & 14.9 & 00.0 & 16.3 & 13.1 & 35.5 & 00.0 & 00.0 & 00.0 & 00.0 \\
\hline 12 & 2 & $40-41$ & 91.1 & 73.8 & 00.0 & 00.0 & 39.0 & 00.0 & 35.1 & 00.0 & 00.0 & 25.7 & 00.0 & 00.0 & 00.0 & 00.0 & 00.0 \\
\hline 12 & 2 & $147-148$ & 91.1 & 73.8 & 00.0 & 00.0 & 18.1 & 00.0 & 14.1 & 00.0 & 7.4 & 11.9 & 48.3 & 00.0 & 00.0 & 00.0 & 00.0 \\
\hline
\end{tabular}


Hole 34 - Continued

\begin{tabular}{|c|c|c|c|c|c|c|c|c|c|c|c|c|c|c|c|c|c|}
\hline Core & Section & $\begin{array}{l}\text { Depth } \\
(\mathrm{cm})\end{array}$ & Diff. & $\begin{array}{c}\% \\
\text { Amorphous }\end{array}$ & Calc. & Dolo. & Quar. & $\begin{array}{l}\text { K- } \\
\text { Feld. }\end{array}$ & Plag. & Kaol. & Mica & Chlo. & Mont. & Pyri. & Bari. & Clin. & Phil. \\
\hline 12 & 6 & $8-9$ & 91.1 & 73.8 & 00.0 & 00.0 & 16.9 & 00.0 & 10.6 & 00.0 & 00.0 & 17.4 & 54.3 & 00.0 & 00.0 & 00.0 & 00.0 \\
\hline 13 & 2 & 10 & 91.3 & 74.4 & 00.0 & 00.0 & 14.4 & 00.0 & 12.9 & 00.0 & 11.7 & 9.5 & 51.3 & 00.0 & 00.0 & 00.0 & 00.0 \\
\hline 13 & 2 & 22 & 91.6 & 75.3 & 00.0 & 00.0 & 20.0 & 00.0 & 10.4 & 00.0 & 00.0 & 15.8 & 53.6 & 00.0 & 00.0 & 00.0 & 00.0 \\
\hline 13 & 2 & 34 & 89.4 & 68.8 & 75.8 & 00.0 & 4.7 & 00.0 & 2.7 & 00.0 & 00.0 & 2.7 & 13.8 & 00.0 & 00.0 & 00.0 & 00.0 \\
\hline 13 & 2 & 60 & 90.3 & 71.5 & 49.7 & 00.0 & 8.8 & 00.0 & 5.4 & 00.0 & 6.2 & 6.7 & 23.0 & 00.0 & 00.0 & 00.0 & 00.0 \\
\hline 13 & 2 & 115 & 91.6 & 75.3 & 00.0 & 00.0 & 14.2 & 00.0 & 14.9 & 00.0 & 23.2 & 7.5 & 17.6 & 22.4 & 00.0 & 00.0 & 00.0 \\
\hline 14 & 2 & $78-79$ & 90.9 & 73.2 & 00.0 & 00.0 & 14.6 & 00.0 & 9.8 & 00.0 & 15.5 & 7.7 & 52.1 & 00.0 & 00.0 & 00.0 & 00.0 \\
\hline 14 & 2 & $128-129$ & 89.7 & 69.7 & 00.0 & 1.7 & 4.3 & 00.0 & 3.0 & 00.0 & 5.8 & 00.0 & 85.1 & 00.0 & 00.0 & 00.0 & 00.0 \\
\hline 15 & 1 & 110 & 90.2 & 71.2 & 00.0 & 00.0 & 6.0 & 00.0 & 3.6 & 00.0 & 7.4 & 00.0 & 80.9 & 00.0 & 00.0 & 2.0 & 00.0 \\
\hline 15 & 2 & 94 & 89.7 & 69.7 & 00.0 & 1.0 & 4.0 & 00.0 & 3.2 & 00.0 & 5.1 & 00.0 & 86.5 & 00.0 & 00.0 & 00.0 & 00.0 \\
\hline 15 & 2 & $122-123$ & 90.9 & 73.2 & 00.0 & 3.5 & 16.1 & 00.0 & 10.6 & 00.0 & 11.7 & 00.0 & 45.7 & 00.0 & 00.0 & 12.2 & 00.0 \\
\hline 16 & 1 & 34 & 90.2 & 71.2 & 00.0 & 3.1 & 10.9 & 00.0 & 8.9 & 00.0 & 10.7 & 00.0 & 48.7 & 00.0 & 00.0 & 17.4 & 00.0 \\
\hline 16 & 1 & 74 & 90.4 & 71.8 & 00.0 & 00.0 & 9.5 & 00.0 & 6.6 & 00.0 & 9.3 & 00.0 & 72.8 & 00.0 & 00.0 & 2.1 & 00.0 \\
\hline 16 & 1 & 80 & 88.7 & 66.8 & 72.7 & 00.0 & 6.8 & 00.0 & 4.9 & 00.0 & 12.1 & 00.0 & 00.0 & 00.0 & 00.0 & 3.4 & 00.0 \\
\hline 16 & 1 & 130 & 90.9 & 73.2 & 9.2 & 00.0 & 14.2 & 00.0 & 9.0 & 00.0 & 14.0 & 00.0 & 51.1 & 00.0 & 00.0 & 2.2 & 00.0 \\
\hline 18 & 1 & $\begin{array}{l}\text { core } \\
\text { catcher }\end{array}$ & 90.1 & 70.9 & 31.2 & 00.0 & 11.7 & 00.0 & 7.8 & 00.0 & 17.2 & 4.2 & 23.0 & 00.0 & 00.0 & 4.7 & 00.0 \\
\hline 18 & 1 & $\begin{array}{l}\text { core } \\
\text { catcher }\end{array}$ & 86.9 & 61.5 & 97.9 & 00.0 & 2.0 & 00.0 & 00.0 & 00.0 & 00.0 & 00.0 & 00.0 & 00.0 & 00.0 & 00.0 & 00.0 \\
\hline \multicolumn{18}{|c|}{ Hole 35} \\
\hline Core & Section & $\begin{array}{l}\text { Depth } \\
\text { (cm) }\end{array}$ & Diff. & $\begin{array}{c}\% \\
\text { Amorphous }\end{array}$ & Calc. & Dolo. & Quar. & $\begin{array}{l}\text { K- } \\
\text { Feld. }\end{array}$ & Plag. & Kaol. & Mica & Chlo. & Mont. & Pyri. & Bari. & Clin. & Phil. \\
\hline 1 & 1 & $24-25$ & 87.3 & 62.6 & 98.0 & 00.0 & 2.0 & 00.0 & 00.0 & 00.0 & 00.0 & 00.0 & 00.0 & 00.0 & 00.0 & 00.0 & 00.0 \\
\hline 1 & 1 & $80-81$ & 89.6 & 69.4 & 00.0 & 00.0 & 39.4 & 00.0 & 24.4 & 4.7 & 25.2 & 6.3 & 00.0 & 00.0 & 00.0 & 00.0 & 00.0 \\
\hline 1 & 2 & $50-51$ & 90.7 & 72.6 & 00.0 & 00.0 & 19.8 & 00.0 & 14.0 & 7.9 & 31.7 & 5.3 & 21.2 & 00.0 & 00.0 & 00.0 & 00.0 \\
\hline
\end{tabular}


Results of X-Ray Diffraction Analysis of Bulk Samples from Leg 5 - Continued

Hole 35 - Continued

\begin{tabular}{|c|c|c|c|c|c|c|c|c|c|c|c|c|c|c|c|c|c|}
\hline Core & Section & $\begin{array}{l}\text { Depth } \\
(\mathrm{cm})\end{array}$ & Diff. & $\begin{array}{c}\% \\
\text { Amorphous }\end{array}$ & Calc. & Dolo. & Quar. & $\begin{array}{l}\text { K- } \\
\text { Feld. }\end{array}$ & Plag. & Kaol. & Mica & Chlo. & Mont. & Pyri. & Bari. & Clin. & Phil. \\
\hline 1 & 2 & $90-91$ & 90.6 & 72.4 & 00.0 & 00.0 & 18.3 & 00.0 & 12.5 & 11.0 & 29.4 & 5.9 & 22.9 & 00.0 & 00.0 & 00.0 & 00.0 \\
\hline 1 & 3 & $22-23$ & 90.7 & 72.6 & 00.0 & 00.0 & 22.4 & 00.0 & 14.8 & 11.6 & 26.9 & 4.6 & 20.0 & 00.0 & 00.0 & 00.0 & 00.0 \\
\hline 1 & 5 & $11-12$ & 90.4 & 71.8 & 00.0 & 00.0 & 24.6 & 00.0 & 16.2 & 10.8 & 33.5 & 5.9 & 8.9 & 00.0 & 00.0 & 00.0 & 00.0 \\
\hline 2 & 1 & $72-73$ & 90.4 & 71.8 & 00.0 & 00.0 & 23.9 & 00.0 & 16.0 & 9.5 & 28.6 & 5.0 & 16.9 & 00.0 & 00.0 & 00.0 & 00.0 \\
\hline 2 & 2 & $23-24$ & 90.4 & 71.8 & 00.0 & 00.0 & 25.4 & 00.0 & 14.5 & 10.2 & 32.5 & 8.1 & 9.2 & 00.0 & 00.0 & 00.0 & 00.0 \\
\hline 2 & 3 & $5-6$ & 90.5 & 72.1 & 00.0 & 00.0 & 28.8 & 00.0 & 14.3 & 13.8 & 32.3 & 7.8 & 00.0 & 00.0 & 00.0 & 00.0 & 00.0 \\
\hline 2 & 3 & $71-72$ & 90.5 & 72.1 & 00.0 & 00.0 & 29.5 & 00.0 & 17.7 & 11.8 & 33.0 & 8.0 & 00.0 & 00.0 & 00.0 & 00.0 & 00.0 \\
\hline 2 & 4 & $13-14$ & 90.5 & 72.1 & 00.0 & 00.0 & 23.1 & 00.0 & 12.9 & 10.2 & 27.7 & 5.5 & 20.6 & 00.0 & 00.0 & 00.0 & 00.0 \\
\hline 2 & 4 & $69-70$ & 90.3 & 71.5 & 00.0 & 00.0 & 28.0 & 00.0 & 16.2 & 14.6 & 35.8 & 5.3 & 00.0 & 00.0 & 00.0 & 00.0 & 00.0 \\
\hline 6 & 2 & $51-52$ & 89.3 & 68.5 & 00.0 & 2.2 & 36.6 & 00.0 & 22.7 & 5.9 & 26.4 & 6.2 & 00.0 & 00.0 & 00.0 & 00.0 & 00.0 \\
\hline 6 & 4 & $10-11$ & 90.3 & 71.5 & 3.7 & 2.3 & 19.4 & 00.0 & 10.3 & 9.3 & 26.4 & 4.1 & 24.3 & 00.0 & 00.0 & 00.0 & 00.0 \\
\hline 6 & 5 & $10-12$ & 90.3 & 71.5 & 4.8 & 2.8 & 23.0 & 00.0 & 11.0 & 11.9 & 29.4 & 4.8 & 12.2 & 00.0 & 00.0 & 00.0 & 00.0 \\
\hline 6 & 6 & $11-12$ & 90.0 & 70.6 & 8.0 & 3.4 & 24.1 & 00.0 & 12.8 & 10.6 & 34.7 & 6.5 & 00.0 & 00.0 & 00.0 & 00.0 & 00.0 \\
\hline 7 & 3 & $18-19$ & 90.0 & 70.6 & 8.0 & 2.9 & 24.3 & 00.0 & 12.9 & 11.7 & 35.0 & 5.1 & 00.0 & 00.0 & 00.0 & 00.0 & 00.0 \\
\hline 7 & 3 & $94-95$ & 89.9 & 70.3 & 00.0 & 2.4 & 23.8 & 00.0 & 12.6 & 9.5 & 43.9 & 7.6 & 00.0 & 00.0 & 00.0 & 00.0 & 00.0 \\
\hline 7 & 4 & $7-8$ & 89.4 & 68.8 & 5.6 & 2.4 & 24.6 & 00.0 & 11.0 & 8.8 & 33.4 & 5.2 & 8.8 & 00.0 & 00.0 & 00.0 & 00.0 \\
\hline 7 & 4 & $74-75$ & 90.1 & 70.9 & 00.0 & 3.1 & 25.8 & 00.0 & 15.5 & 11.4 & 37.2 & 7.0 & 00.0 & 00.0 & 00.0 & 00.0 & 00.0 \\
\hline 8 & 1 & $105 \cdot 106$ & 90.4 & 71.8 & 7.4 & 3.3 & 25.4 & 00.0 & 14.0 & 13.2 & 28.5 & 8.1 & 00.0 & 00.0 & 00.0 & 00.0 & 00.0 \\
\hline 9 & 1 & $88-89$ & 90.3 & 71.5 & 00.0 & 1.8 & 20.5 & 00.0 & 11.7 & 13.9 & 28.5 & 5.3 & 18.2 & 00.0 & 00.0 & 00.0 & 00.0 \\
\hline 9 & 1 & $109-110$ & 89.9 & 70.3 & 5.6 & 2.7 & 24.3 & 00.0 & 12.4 & 13.6 & 33.7 & 7.8 & 00.0 & 00.0 & 00.0 & 00.0 & 00.0 \\
\hline 9 & 2 & $9-10$ & 89.0 & 67.6 & 3.5 & 2.4 & 27.1 & 00.0 & 14.6 & 8.7 & 24.4 & 4.9 & 14.6 & 00.0 & 00.0 & 00.0 & 00.0 \\
\hline 9 & 2 & $74-75$ & 89.8 & 70.0 & 6.2 & 2.7 & 24.6 & 00.0 & 12.1 & 15.8 & 32.3 & 6.4 & 00.0 & 00.0 & 00.0 & 00.0 & 00.0 \\
\hline 9 & 3 & $6-7$ & 89.8 & 70.0 & 8.0 & 2.7 & 24.3 & 00.0 & 12.6 & 12.6 & 00.0 & 00.0 & 00.0 & 00.0 & 00.0 & 00.0 & 00.0 \\
\hline 9 & 3 & $75-76$ & 89.7 & 69.7 & 4.9 & 2.5 & 21.2 & 00.0 & 10.2 & 9.3 & 26.1 & 6.8 & 00.0 & 00.0 & 00.0 & 00.0 & 00.0 \\
\hline 11 & 6 & $9-10$ & 89.7 & 69.7 & 5.8 & 2.8 & 25.0 & 00.0 & 12.1 & 15.0 & 32.8 & 6.5 & 00.0 & 00.0 & 00.0 & 00.0 & 00.0 \\
\hline 11 & 6 & $74-75$ & 89.2 & 68.2 & 00.0 & 2.6 & 29.2 & 00.0 & 14.3 & 14.0 & 33.6 & 6.1 & 00.0 & 00.0 & 00.0 & 00.0 & 00.0 \\
\hline
\end{tabular}


Results of X-Ray Diffraction Analysis of Bulk Samples from Leg 5 - Continued

Hole 35 - Continued

\begin{tabular}{|c|c|c|c|c|c|c|c|c|c|c|c|c|c|c|c|c|c|}
\hline Core & Section & $\begin{array}{l}\text { Depth } \\
(\mathrm{cm})\end{array}$ & Diff. & $\begin{array}{c}\% \\
\text { Amorphous }\end{array}$ & Calc. & Dolo. & Quar. & $\begin{array}{l}\text { K- } \\
\text { Feld. }\end{array}$ & Plag. & Kaol. & Mica & Chlo. & Mont. & Pyri. & Bari. & Clin. & Phil. \\
\hline 12 & 6 & $56-57$ & 89.2 & 68.2 & 6.7 & 3.0 & 27.7 & 00.0 & 15.0 & 11.1 & 29.6 & 7.2 & 00.0 & 00.0 & 00.0 & 00.0 & 00.0 \\
\hline 12 & 6 & $140-141$ & 88.3 & 70.0 & 7.5 & 00.0 & 21.4 & 00.0 & 9.0 & 20.6 & 35.2 & 6.2 & 00.0 & 00.0 & 00.0 & 00.0 & 00.0 \\
\hline 13 & 4 & $50-51$ & 87.7 & 69.7 & 7.8 & 00.0 & 20.5 & 00.0 & 11.7 & 20.5 & 33.6 & 5.9 & 00.0 & 00.0 & 00.0 & 00.0 & 00.0 \\
\hline 13 & 4 & $106-107$ & 87.7 & 69.7 & 00.0 & 00.0 & 20.2 & 00.0 & 12.1 & 23.4 & 36.3 & 8.1 & 00.0 & 00.0 & 00.0 & 00.0 & 00.0 \\
\hline 14 & 4 & $9-10$ & 89.8 & 70.0 & 00.0 & 2.4 & 24.5 & 00.0 & 12.7 & 13.7 & 36.3 & 10.3 & 00.0 & 00.0 & 00.0 & 00.0 & 00.0 \\
\hline 14 & 4 & 84.85 & 87.5 & 69.1 & 00.0 & 00.0 & 16.7 & 00.0 & 11.0 & 15.3 & 36.8 & 8.3 & 11.8 & 00.0 & 00.0 & 00.0 & 00.0 \\
\hline 14 & 4 & $140-141$ & 88.0 & 64.7 & 00.0 & 00.0 & 21.4 & 00.0 & 16.2 & 20.5 & 33.3 & 8.5 & 00.0 & 00.0 & 00.0 & 00.0 & 00.0 \\
\hline 15 & 4 & $6-7$ & 87.6 & 63.5 & 00.0 & 00.0 & 15.6 & 00.0 & 11.8 & 12.5 & 34.4 & 6.2 & 19.5 & 00.0 & 00.0 & 00.0 & 00.0 \\
\hline 15 & 4 & $74-75$ & 87.5 & 63.2 & 00.0 & 00.0 & 15.6 & 00.0 & 12.6 & 14.3 & 31.9 & 6.2 & 19.4 & 00.0 & 00.0 & 00.0 & 00.0 \\
\hline 16 & 2 & $19-20$ & 87.3 & 62.6 & 00.0 & 00.0 & 28.7 & 00.0 & 16.4 & 00.0 & 19.0 & 00.0 & 35.9 & 00.0 & 00.0 & 00.0 & 00.0 \\
\hline 16 & 2 & $90-91$ & 87.1 & 62.1 & 00.0 & 00.0 & 31.0 & 00.0 & 18.6 & 00.0 & 17.7 & 5.0 & 27.6 & 00.0 & 00.0 & 00.0 & 00.0 \\
\hline \multicolumn{18}{|c|}{ Hole 36} \\
\hline Core & Section & $\begin{array}{l}\text { Depth } \\
(\mathrm{cm})\end{array}$ & Diff. & $\begin{array}{c}\% \\
\text { Amorphous }\end{array}$ & Calc. & Dolo. & Quar. & $\begin{array}{l}\text { K- } \\
\text { Feld. }\end{array}$ & Plag. & Kaol. & Mica & Chlo. & Mont. & Pyri. & Bari. & Clin. & Phil. \\
\hline 1 & 1 & $10-11$ & 91.2 & 74.1 & 00.0 & 00.0 & 29.7 & 00.0 & 14.2 & 11.9 & 36.5 & 7.7 & 00.0 & 00.0 & 00.0 & 00.0 & 00.0 \\
\hline 1 & 1 & $75-76$ & 89.4 & 68.8 & 27.0 & 00.0 & 25.4 & 00.0 & 11.4 & 5.1 & 20.9 & 10.2 & 00.0 & 00.0 & 00.0 & 00.0 & 00.0 \\
\hline 4 & 3 & $20-22$ & 88.5 & 66.2 & 54.1 & 00.0 & 13.5 & 00.0 & 6.8 & 4.5 & 16.0 & 5.1 & 00.0 & 00.0 & 00.0 & 00.0 & 00.0 \\
\hline 5 & 3 & $70-71$ & 88.0 & 64.7 & 63.6 & 00.0 & 9.9 & 00.0 & 4.6 & 00.0 & 00.0 & 5.3 & 00.0 & 16.6 & 00.0 & 00.0 & 00.0 \\
\hline 6 & 1 & $90-91$ & 87.8 & 64.1 & 76.8 & 00.0 & 8.0 & 00.0 & 4.4 & 00.0 & 6.4 & 4.4 & 00.0 & 00.0 & 00.0 & 00.0 & 00.0 \\
\hline 6 & 3 & $75-76$ & 89.9 & 70.3 & 31.5 & 00.0 & 23.5 & 00.0 & 11.3 & 5.6 & 19.3 & 8.7 & 00.0 & 00.0 & 00.0 & 00.0 & 00.0 \\
\hline 6 & 4 & $27-28$ & 88.5 & 66.2 & 73.6 & 00.0 & 12.2 & 00.0 & 8.0 & 00.0 & 00.0 & 6.1 & 00.0 & 00.0 & 00.0 & 00.0 & 00.0 \\
\hline 6 & 4 & $110-111$ & 90.5 & 72.1 & 00.0 & 00.0 & 35.8 & 00.0 & 19.0 & 10.0 & 23.6 & 11.5 & 00.0 & 00.0 & 00.0 & 00.0 & 00.0 \\
\hline 6 & 6 & $85-86$ & 88.1 & 65.0 & 73.3 & 00.0 & 8.8 & 00.0 & 4.2 & 00.0 & 9.5 & 4.2 & 00.0 & 00.0 & 00.0 & 00.0 & 00.0 \\
\hline 8 & 1 & $75-76$ & 89.9 & 70.3 & 24.8 & 00.0 & 31.4 & 00.0 & 14.2 & 00.0 & 17.9 & 11.6 & 00.0 & 00.0 & 00.0 & 00.0 & 00.0 \\
\hline
\end{tabular}


Results of X-Ray Diffraction Analysis of Bulk Samples from Leg 5 - Continued

Hole 36 - Continued

\begin{tabular}{|c|c|c|c|c|c|c|c|c|c|c|c|c|c|c|c|c|c|}
\hline Core & Section & $\begin{array}{l}\text { Depth } \\
(\mathrm{cm})\end{array}$ & Diff. & $\begin{array}{c}\% \\
\text { Amorphous }\end{array}$ & Calc. & Dolo. & Quar. & $\begin{array}{l}\text { K- } \\
\text { Feld. }\end{array}$ & Plag. & Kaol. & Mica & Chlo. & Mont. & Pyri. & Bari. & Clin. & Phil. \\
\hline 8 & 1 & $83-84$ & 90.3 & 71.5 & 00.0 & 00.0 & 40.3 & 00.0 & 18.5 & 12.9 & 23.0 & 5.2 & 00.0 & 00.0 & 00.0 & 00.0 & 00.0 \\
\hline 8 & 2 & $11-12$ & 88.6 & 66.5 & 64.9 & 00.0 & 13.2 & 00.0 & 6.4 & 00.0 & 11.1 & 4.4 & 00.0 & 00.0 & 00.0 & 00.0 & 00.0 \\
\hline 8 & 3 & $68-69$ & 89.8 & 70.0 & 28.6 & 00.0 & 27.0 & 00.0 & 14.3 & 00.0 & 22.2 & 7.8 & 00.0 & 00.0 & 00.0 & 00.0 & 00.0 \\
\hline 8 & 4 & $57-58$ & 90.3 & 74.4 & 11.2 & 00.0 & 44.6 & 00.0 & 22.7 & 21.4 & 00.0 & 00.0 & 00.0 & 00.0 & 00.0 & 00.0 & 00.0 \\
\hline 8 & 5 & $108-109$ & 89.7 & 69.7 & 19.0 & 00.0 & 24.7 & 00.0 & 11.1 & 2.0 & 20.2 & 9.9 & 13.1 & 00.0 & 00.0 & 00.0 & 00.0 \\
\hline 8 & 6 & $10-11$ & 89.9 & 70.3 & 20.0 & 00.0 & 24.7 & 00.0 & 10.9 & 3.0 & 14.1 & 9.9 & 17.5 & 00.0 & 00.0 & 00.0 & 00.0 \\
\hline 8 & 6 & $75-76$ & 90.1 & 70.9 & 24.2 & 00.0 & 22.8 & 00.0 & 11.0 & 2.7 & 13.0 & 5.9 & 20.3 & 00.0 & 00.0 & 00.0 & 00.0 \\
\hline 9 & 2 & $60-61$ & 90.2 & 71.2 & 10.0 & 00.0 & 30.2 & 00.0 & 15.4 & 00.0 & 22.4 & 11.2 & 00.0 & 00.0 & 10.9 & 00.0 & 00.0 \\
\hline 9 & 3 & $39-40$ & 90.3 & 71.5 & 12.3 & 00.0 & 30.7 & 00.0 & 14.7 & 00.0 & 22.7 & 10.4 & 00.0 & 00.0 & 9.2 & 00.0 & 00.0 \\
\hline 10 & 1 & $29-30$ & 90.1 & 70.9 & 30.2 & 00.0 & 14.9 & 00.0 & 7.5 & 00.0 & 11.6 & 5.3 & 25.2 & 00.0 & 5.2 & 00.0 & 00.0 \\
\hline 10 & 1 & $75-76$ & 89.8 & 70.0 & 40.8 & 00.0 & 12.7 & 00.0 & 6.4 & 00.0 & 8.7 & 6.2 & 18.9 & 00.0 & 6.4 & 00.0 & 00.0 \\
\hline 10 & 2 & $11-12$ & 89.2 & 68.2 & 57.1 & 00.0 & 11.9 & 00.0 & 6.8 & 00.0 & 00.0 & 3.9 & 15.8 & 00.0 & 4.5 & 00.0 & 00.0 \\
\hline 10 & 2 & $75-76$ & 90.3 & 71.5 & 29.5 & 00.0 & 22.5 & 00.0 & 10.1 & 3.6 & 20.3 & 5.8 & 00.0 & 00.0 & 8.1 & 00.0 & 00.0 \\
\hline 10 & 3 & $6-7$ & 90.3 & 71.5 & 32.8 & 00.0 & 22.8 & 00.0 & 11.0 & 2.7 & 13.0 & 7.7 & 00.0 & 00.0 & 9.8 & 00.0 & 00.0 \\
\hline 10 & 3 & $74-75$ & 90.3 & 71.5 & 00.0 & 00.0 & 27.2 & 00.0 & 13.3 & 00.0 & 20.1 & 7.1 & 24.2 & 00.0 & 8.2 & 00.0 & 00.0 \\
\hline 10 & 4 & $10-11$ & 89.6 & 69.4 & 38.2 & 00.0 & 11.6 & 00.0 & 6.0 & 00.0 & 9.8 & 4.8 & 24.9 & 00.0 & 4.8 & 00.0 & 00.0 \\
\hline 10 & 4 & $70-71$ & 89.1 & 67.9 & 50.5 & 00.0 & 14.5 & 00.0 & 8.2 & 00.0 & 8.7 & 4.2 & 13.9 & 00.0 & 00.0 & 00.0 & 00.0 \\
\hline 10 & 5 & $10-11$ & 89.2 & 68.2 & 50.5 & 00.0 & 15.0 & 00.0 & 5.5 & 00.0 & 10.8 & 4.2 & 13.9 & 00.0 & 00.0 & 00.0 & 00.0 \\
\hline 10 & 5 & $75-76$ & 89.1 & 67.9 & 55.6 & 00.0 & 11.0 & 00.0 & 6.7 & 2.3 & 00.0 & 3.8 & 15.4 & 00.0 & 00.0 & 00.0 & 00.0 \\
\hline 10 & 6 & $10-11$ & 90.6 & 72.4 & 19.4 & 00.0 & 18.3 & 00.0 & 9.3 & 1.5 & 15.0 & 5.8 & 22.8 & 00.0 & 7.9 & 00.0 & 00.0 \\
\hline 10 & 6 & $75-76$ & 90.5 & 72.1 & 17.6 & 00.0 & 16.2 & 00.0 & 8.7 & 00.0 & 13.3 & 6.0 & 31.7 & 00.0 & 6.5 & 00.0 & 00.0 \\
\hline 11 & 2 & $10-11$ & 90.8 & 72.9 & 00.0 & 00.0 & 28.2 & 00.0 & 15.0 & 3.4 & 18.8 & 9.1 & 25.3 & 00.0 & 00.0 & 00.0 & 00.0 \\
\hline 11 & 2 & $75-76$ & 89.9 & 70.3 & 15.0 & 00.0 & 28.8 & 00.0 & 17.3 & 3.0 & 00.0 & 9.8 & 25.6 & 00.0 & 00.0 & 00.0 & 00.0 \\
\hline 11 & 3 & $15-16$ & 90.3 & 71.5 & 11.1 & 00.0 & 29.3 & 00.0 & 15.5 & 5.9 & 24.0 & 7.0 & 00.0 & 00.0 & 7.0 & 00.0 & 00.0 \\
\hline 11 & 3 & $75-76$ & 90.4 & 71.8 & 7.3 & 00.0 & 23.7 & 00.0 & 13.5 & 00.0 & 21.3 & 8.0 & 21.1 & 00.0 & 5.0 & 00.0 & 00.0 \\
\hline 11 & 4 & $10-11$ & 90.3 & 71.5 & 15.1 & 00.0 & 26.1 & 00.0 & 14.9 & 3.1 & 17.2 & 9.7 & 13.8 & 00.0 & 00.0 & 00.0 & 00.0 \\
\hline
\end{tabular}


Hole 36 - Continued

\begin{tabular}{|c|c|c|c|c|c|c|c|c|c|c|c|c|c|c|c|c|c|}
\hline Core & Section & $\begin{array}{l}\text { Depth } \\
\text { (cm) }\end{array}$ & Diff. & $\begin{array}{c}\% \\
\text { Amorphous }\end{array}$ & Calc. & Dolo. & Quar. & $\begin{array}{l}\text { K- } \\
\text { Feld. }\end{array}$ & Plag. & Kaol. & Mica & Chlo. & Mont. & Pyri. & Bari. & Clin. & Phil. \\
\hline 11 & 4 & $77-78$ & 89.9 & 70.3 & 26.4 & 00.0 & 28.6 & 00.0 & 15.5 & 6.9 & 00.0 & 7.4 & 15.2 & 00.0 & 00.0 & 00.0 & 00.0 \\
\hline 11 & 5 & $10-11$ & 90.2 & 71.2 & 18.2 & 00.0 & 24.9 & 00.0 & 12.2 & 00.0 & 00.0 & 10.5 & 26.7 & 00.0 & 7.5 & 00.0 & 00.0 \\
\hline 11 & 5 & $90-91$ & 90.2 & 71.2 & 9.5 & 00.0 & 26.4 & 00.0 & 13.4 & 4.2 & 12.9 & 6.9 & 18.7 & 00.0 & 7.9 & 00.0 & 00.0 \\
\hline 11 & 6 & $39-40$ & 89.7 & 69.7 & 45.2 & 00.0 & 17.2 & 00.0 & 9.6 & 13.4 & 00.0 & 6.1 & 8.5 & 00.0 & 00.0 & 00.0 & 00.0 \\
\hline 11 & 6 & $50-51$ & 90.1 & 70.9 & 10.5 & 00.0 & 25.1 & 00.0 & 12.8 & 6.0 & 16.5 & 5.3 & 17.8 & 00.0 & 6.0 & 00.0 & 00.0 \\
\hline 12 & 1 & $10-11$ & 89.7 & 69.7 & 50.8 & 00.0 & 19.6 & 00.0 & 19.8 & 00.0 & 13.0 & 00.0 & 6.9 & 00.0 & 00.0 & 00.0 & 00.0 \\
\hline 12 & 1 & $76-77$ & 89.6 & 69.4 & 38.5 & 00.0 & 15.0 & 00.0 & 7.4 & 00.0 & 16.4 & 8.4 & 14.2 & 00.0 & 00.0 & 00.0 & 00.0 \\
\hline 12 & 2 & $10-11$ & 88.9 & 67.4 & 50.9 & 00.0 & 10.6 & 00.0 & 6.1 & 00.0 & 6.6 & 6.9 & 18.8 & 00.0 & 00.0 & 00.0 & 00.0 \\
\hline 12 & 2 & $66-67$ & 89.2 & 68.2 & 50.3 & 00.0 & 15.2 & 00.0 & 7.8 & 00.0 & 21.8 & 8.4 & 00.0 & 00.0 & 5.5 & 00.0 & 00.0 \\
\hline 12 & 3 & $10-11$ & 89.3 & 68.5 & 48.7 & 00.0 & 14.5 & 00.0 & 6.8 & 00.0 & 00.0 & 7.4 & 18.0 & 00.0 & 4.6 & 00.0 & 00.0 \\
\hline 12 & 3 & $101-102$ & 89.2 & 68.2 & 46.3 & 00.0 & 13.7 & 00.0 & 7.2 & 00.0 & 9.9 & 3.8 & 17.1 & 00.0 & 00.0 & 00.0 & 00.0 \\
\hline 12 & 4 & $15-16$ & 89.0 & 67.6 & 43.7 & 00.0 & 12.8 & 00.0 & 5.7 & 00.0 & 9.3 & 4.1 & 20.3 & 00.0 & 4.1 & 00.0 & 00.0 \\
\hline 12 & 4 & $76-77$ & 88.7 & 66.8 & 57.1 & 00.0 & 11.3 & 00.0 & 5.6 & 00.0 & 00.0 & 4.8 & 21.1 & 00.0 & 00.0 & 00.0 & 00.0 \\
\hline 12 & 5 & $15-16$ & 88.5 & 66.2 & 63.2 & 00.0 & 8.6 & 00.0 & 5.6 & 00.0 & 00.0 & 5.3 & 17.1 & 00.0 & 00.0 & 00.0 & 00.0 \\
\hline 12 & 5 & $75-76$ & 88.6 & 66.5 & 56.6 & 00.0 & 9.7 & 00.0 & 4.4 & 00.0 & 9.7 & 3.8 & 15.6 & 00.0 & 00.0 & 00.0 & 00.0 \\
\hline 12 & 6 & $15-16$ & 90.2 & 71.2 & 37.2 & 00.0 & 7.0 & 00.0 & 9.6 & 00.0 & 00.0 & 5.0 & 41.5 & 00.0 & 00.0 & 00.0 & 00.0 \\
\hline 12 & 6 & $25-26$ & 89.0 & 67.6 & 69.8 & 00.0 & 5.8 & 00.0 & 5.1 & 00.0 & 00.0 & 00.0 & 19.3 & 00.0 & 00.0 & 00.0 & 00.0 \\
\hline 12 & 6 & $65-66$ & 88.4 & 65.9 & 73.0 & 00.0 & 7.2 & 00.0 & 4.2 & 00.0 & 12.5 & 3.0 & 00.0 & 00.0 & 00.0 & 00.0 & 00.0 \\
\hline 13 & 2 & $10-11$ & 90.6 & 72.4 & 29.0 & 00.0 & 20.1 & 00.0 & 9.8 & 00.0 & 00.0 & 5.2 & 35.8 & 00.0 & 00.0 & 00.0 & 00.0 \\
\hline 13 & 2 & $78-79$ & 90.5 & 72.1 & 8.8 & 00.0 & 23.0 & 00.0 & 11.8 & 1.8 & 15.2 & 6.7 & 32.7 & 00.0 & 00.0 & 00.0 & 00.0 \\
\hline 13 & 3 & $10-11$ & 90.7 & 72.6 & 21.9 & 00.0 & 10.7 & 00.0 & 2.6 & 00.0 & 00.0 & 7.2 & 50.6 & 00.0 & 6.1 & 00.0 & 00.0 \\
\hline 13 & 3 & $93-94$ & 90.8 & 72.9 & 00.0 & 00.0 & 32.9 & 00.0 & 18.1 & 18.4 & 18.8 & 00.0 & 00.0 & 00.0 & 11.8 & 00.0 & 00.0 \\
\hline 13 & 4 & $136-137$ & 90.9 & 73.2 & 00.0 & 00.0 & 14.4 & 00.0 & 21.7 & 5.8 & 11.8 & 7.6 & 38.6 & 00.0 & 00.0 & 00.0 & 00.0 \\
\hline
\end{tabular}


Results of X-Ray Diffraction Analysis of Bulk Samples from Leg 5 - Continued

Hole 37

\begin{tabular}{|c|c|c|c|c|c|c|c|c|c|c|c|c|c|c|c|c|c|}
\hline Core & Section & $\begin{array}{l}\text { Depth } \\
(\mathrm{cm})\end{array}$ & Diff. & $\begin{array}{c}\% \\
\text { Amorphous }\end{array}$ & Calc. & Dolo. & Quar. & $\begin{array}{l}\text { K- } \\
\text { Feld. }\end{array}$ & Plag. & Kaol. & Mica & Chlo. & Mont. & Pyri. & Bari. & Clin. & Phil. \\
\hline 1 & 3 & $50-51$ & 88.7 & 69.7 & 00.0 & 00.0 & 29.3 & 00.0 & 10.6 & 7.0 & 36.1 & 5.3 & 00.0 & 00.0 & 11.1 & 00.0 & 00.0 \\
\hline 1 & 4 & $62-63$ & 90.0 & 70.6 & 00.0 & 00.0 & 13.2 & 00.0 & 00.0 & 00.0 & 21.1 & 3.4 & 00.0 & 00.0 & 15.3 & 00.0 & 47.0 \\
\hline 2 & 1 & 64-65 & 89.2 & 68.2 & 00.0 & 00.0 & 33.3 & 00.0 & 00.0 & 2.8 & 36.4 & 3.0 & 24.9 & 00.0 & 16.3 & 00.0 & 16.6 \\
\hline 2 & 2 & $34-35$ & 89.9 & 70.3 & 00.0 & 00.0 & 11.8 & 00.0 & 1.4 & 00.0 & 14.6 & 4.4 & 00.0 & 00.0 & 15.1 & 00.0 & 52.7 \\
\hline 2 & 2 & $92-93$ & 90.7 & 72.6 & 00.0 & 00.0 & 7.2 & 00.0 & 00.0 & 00.0 & 00.0 & 10.5 & 00.0 & 00.0 & 11.6 & 00.0 & 70.8 \\
\hline 2 & 3 & $15-16$ & 90.4 & 71.8 & 00.0 & 00.0 & 16.7 & 00.0 & 7.5 & 11.3 & 10.7 & 11.0 & 00.0 & 00.0 & 25.0 & 00.0 & 17.8 \\
\hline 2 & 4 & $14-15$ & 90.6 & 72.4 & 00.0 & 00.0 & 15.4 & 00.0 & 8.2 & 00.0 & 19.0 & 8.2 & 33.0 & 00.0 & 16.3 & 00.0 & 00.0 \\
\hline 2 & 5 & $23-24$ & 90.8 & 72.9 & 00.0 & 00.0 & 25.8 & 00.0 & 13.7 & 00.0 & 31.8 & 6.7 & 00.0 & 00.0 & 22.0 & 00.0 & 00.0 \\
\hline 2 & 6 & $19-20$ & 90.9 & 73.2 & 00.0 & 00.0 & 24.2 & 00.0 & 12.6 & 00.0 & 38.8 & 9.6 & 00.0 & 00.0 & 14.9 & 00.0 & 00.0 \\
\hline 3 & 1 & $10-12$ & 91.3 & 74.4 & 00.0 & 00.0 & 38.4 & 00.0 & 23.0 & 38.4 & 00.0 & 00.0 & 00.0 & 00.0 & 00.0 & 00.0 & 00.0 \\
\hline 3 & 2 & $21-22$ & 91.5 & 75.0 & 00.0 & 00.0 & 25.4 & 00.0 & 30.5 & 30.5 & 00.0 & 13.4 & 00.0 & 00.0 & 00.0 & 00.0 & 00.0 \\
\hline 3 & 2 & $59-60$ & 91.4 & 74.7 & 00.0 & 00.0 & 13.9 & 9.2 & 4.2 & 00.0 & 22.8 & 5.6 & 00.0 & 00.0 & 00.0 & 00.0 & 44.4 \\
\hline 3 & 3 & NDG & 91.3 & 74.4 & 00.0 & 00.0 & 16.1 & 00.0 & 21.7 & 29.0 & 33.0 & 00.0 & 00.0 & 00.0 & 00.0 & 00.0 & 00.0 \\
\hline 3 & 3 & $110-111$ & 91.1 & 73.8 & 00.0 & 00.0 & 12.9 & 00.0 & 25.8 & 17.2 & 13.6 & 00.0 & 30.6 & 00.0 & 00.0 & 00.0 & 00.0 \\
\hline 3 & 4 & $42-43$ & 91.1 & 73.8 & 00.0 & 00.0 & 28.6 & 00.0 & 42.8 & 5.7 & 00.0 & 22.8 & 00.0 & 00.0 & 00.0 & 00.0 & 00.0 \\
\hline 3 & 5 & $65-66$ & 91.7 & 75.6 & 00.0 & 00.0 & 20.5 & 00.0 & 43.8 & 4.7 & 00.0 & 31.0 & 00.0 & 00.0 & 00.0 & 00.0 & 00.0 \\
\hline 3 & 6 & $33-34$ & 91.4 & 74.7 & 00.0 & 00.0 & 31.6 & 00.0 & 42.7 & 8.9 & 00.0 & 16.8 & 00.0 & 00.0 & 00.0 & 00.0 & 00.0 \\
\hline 4 & 2 & $66-67$ & 91.3 & 74.4 & 00.0 & 00.0 & 14.1 & 00.0 & 38.5 & 00.0 & 00.0 & 47.4 & 00.0 & 00.0 & 00.0 & 00.0 & 00.0 \\
\hline 4 & 2 & $81-82$ & 91.1 & 100.0 & 00.0 & 00.0 & 00.0 & 00.0 & 00.0 & 00.0 & 00.0 & 00.0 & 00.0 & 00.0 & 00.0 & 00.0 & 00.0 \\
\hline 4 & 3 & $119-120$ & 90.1 & 100.0 & 00.0 & 00.0 & 00.0 & 00.0 & 00.0 & 00.0 & 00.0 & 00.0 & 00.0 & 00.0 & 00.0 & 00.0 & 00.0 \\
\hline 4 & 4 & $2-3$ & 89.9 & 100.0 & 00.0 & 00.0 & 00.0 & 00.0 & 00.0 & 00.0 & 00.0 & 00.0 & 00.0 & 00.0 & 00.0 & 00.0 & 00.0 \\
\hline 4 & 4 & $69-70$ & 91.0 & 100.0 & 00.0 & 00.0 & 00.0 & 00.0 & 00.0 & 00.0 & 00.0 & 00.0 & 00.0 & 00.0 & 00.0 & 00.0 & 00.0 \\
\hline 4 & 5 & $3-4$ & 91.5 & 100.0 & 00.0 & 00.0 & 00.0 & 00.0 & 00.0 & 00.0 & 00.0 & 00.0 & 00.0 & 00.0 & 00.0 & 00.0 & 00.0 \\
\hline 4 & 5 & $120-121$ & 91.0 & 100.0 & 00.0 & 00.0 & 00.0 & 00.0 & 00.0 & 00.0 & 00.0 & 00.0 & 00.0 & 00.0 & 00.0 & 00.0 & 00.0 \\
\hline
\end{tabular}


Hole 38

\begin{tabular}{|c|c|c|c|c|c|c|c|c|c|c|c|c|c|c|c|c|c|}
\hline Core & Section & $\begin{array}{l}\text { Depth } \\
(\mathrm{cm})\end{array}$ & Diff. & $\begin{array}{c}\% \\
\text { Amorphous }\end{array}$ & Calc. & Dolo. & Quar. & $\begin{array}{l}\text { K- } \\
\text { Feld. }\end{array}$ & Plag. & Kaol. & Mica & Chlo. & Mont. & Pyri. & Bari. & Clin. & Phil. \\
\hline 2 & 3 & $10-11$ & 89.3 & 68.5 & 00.0 & 00.0 & 30.4 & 00.0 & 10.6 & 8.5 & 45.0 & 5.5 & 00.0 & 00.0 & 00.0 & 00.0 & 00.0 \\
\hline 2 & 3 & $75-76$ & 89.6 & 69.4 & 00.0 & 00.0 & 25.3 & 00.0 & 9.1 & 6.1 & 41.5 & 4.6 & 13.4 & 00.0 & 00.0 & 00.0 & 00.0 \\
\hline 2 & 4 & $65-66$ & 89.8 & 70.0 & 00.0 & 00.0 & 27.8 & 00.0 & 10.9 & 6.7 & 47.9 & 6.7 & 00.0 & 00.0 & 00.0 & 00.0 & 00.0 \\
\hline 2 & 4 & $100-101$ & 89.8 & 70.0 & 00.0 & 00.0 & 28.6 & 00.0 & 10.6 & 16.0 & 44.7 & 00.0 & 00.0 & 00.0 & 00.0 & 00.0 & 00.0 \\
\hline 2 & 5 & $30-31$ & 89.7 & 69.7 & 00.0 & 00.0 & 32.0 & 00.0 & 11.5 & 5.1 & 44.6 & 6.7 & 00.0 & 00.0 & 00.0 & 00.0 & 00.0 \\
\hline 2 & 6 & $18-19$ & 90.0 & 70.6 & 00.0 & 00.0 & 28.0 & 00.0 & 11.5 & 9.0 & 43.7 & 7.8 & 00.0 & 00.0 & 00.0 & 00.0 & 00.0 \\
\hline 2 & 6 & $139-140$ & 91.1 & 73.8 & 00.0 & 00.0 & 21.2 & 00.0 & 11.7 & 00.0 & 20.8 & 8.5 & 37.8 & 00.0 & 00.0 & 00.0 & 00.0 \\
\hline 3 & 1 & $13-14$ & 91.1 & 73.8 & 00.0 & 00.0 & 23.0 & 00.0 & 24.1 & 00.0 & 37.7 & 15.2 & 0.00 & 00.0 & 00.0 & 00.0 & 00.0 \\
\hline 3 & 3 & $10-11$ & 91.2 & 74.1 & 00.0 & 00.0 & 28.8 & 00.0 & 30.2 & 31.1 & 00.0 & 9.8 & 00.0 & 00.0 & 00.0 & 00.0 & 00.0 \\
\hline 3 & 4 & $10-11$ & 91.1 & 73.8 & 00.0 & 00.0 & 10.6 & 00.0 & 00.0 & 00.0 & 15.9 & 5.3 & 00.0 & 00.0 & 00.0 & 00.0 & 68.2 \\
\hline 3 & 5 & $86-87$ & 89.7 & 69.7 & 00.0 & 00.0 & 00.0 & 00.0 & 00.0 & 00.0 & 00.0 & 00.0 & 00.0 & 00.0 & 00.0 & 00.0 & 100.0 \\
\hline 3 & 6 & $22-23$ & 91.4 & 74.7 & 00.0 & 00.0 & 16.4 & 00.0 & 00.0 & 00.0 & 00.0 & 00.0 & 00.0 & 00.0 & 00.0 & 00.0 & 83.6 \\
\hline 4 & 2 & $78-79$ & 90.6 & 72.4 & 00.0 & 00.0 & 5.9 & 00.0 & 00.0 & 00.0 & 25.3 & 00.0 & 00.0 & 00.0 & 00.0 & 00.0 & 68.8 \\
\hline 4 & 3 & $60-61$ & 90.9 & 73.2 & 00.0 & 00.0 & 9.7 & 00.0 & 00.0 & 00.0 & 00.0 & 00.0 & 00.0 & 00.0 & 00.0 & 00.0 & 90.3 \\
\hline 4 & 4 & $75-76$ & 90.5 & 72.1 & 00.0 & 00.0 & 8.0 & 00.0 & 00.0 & 00.0 & 17.5 & 4.6 & 00.0 & 00.0 & 00.0 & 00.0 & 69.9 \\
\hline 4 & 5 & $74-75$ & 90.9 & 73.2 & 00.0 & 00.0 & 8.5 & 00.0 & 00.0 & 4.9 & 00.0 & 00.0 & 00.0 & 00.0 & 00.0 & 00.0 & 86.6 \\
\hline 5 & 1 & $79-80$ & 91.1 & 73.8 & 00.0 & 00.0 & 17.7 & 00.0 & 27.9 & 29.8 & 00.0 & 24.6 & 00.0 & 00.0 & 00.0 & 00.0 & 00.0 \\
\hline 5 & 1 & $132-133$ & 90.5 & 72.1 & 00.0 & 00.0 & 5.8 & 1.6 & 12.0 & 29.6 & 22.3 & 00.0 & 00.0 & 00.0 & 00.0 & 00.0 & 28.6 \\
\hline 5 & 3 & $75-76$ & 91.2 & 100.0 & 00.0 & 00.0 & 00.0 & 00.0 & 00.0 & 00.0 & 00.0 & 00.0 & 00.0 & 00.0 & 00.0 & 00.0 & 00.0 \\
\hline 6 & 1 & $10-11$ & 88.1 & 65.0 & 100.0 & 00.0 & 00.0 & 00.0 & 00.0 & 00.0 & 00.0 & 00.0 & 00.0 & 00.0 & 00.0 & 00.0 & 00.0 \\
\hline 6 & 4 & $140-141$ & 88.4 & 65.9 & 100.0 & 00.0 & 00.0 & 00.0 & 00.0 & 00.0 & 00.0 & 00.0 & 00.0 & 00.0 & 00.0 & 00.0 & 00.0 \\
\hline 6 & 5 & $11-13$ & 87.8 & 64.1 & 100.0 & 00.0 & 00.0 & 00.0 & 00.0 & 00.0 & 00.0 & 00.0 & 00.0 & 00.0 & 00.0 & 00.0 & 00.0 \\
\hline
\end{tabular}


Results of X-Ray Diffraction Analysis of Bulk Samples from Leg 5 - Continued

Hole 39

\begin{tabular}{|c|c|c|c|c|c|c|c|c|c|c|c|c|c|c|c|c|c|}
\hline Core & Section & $\begin{array}{l}\text { Depth } \\
\text { (cm) }\end{array}$ & Diff. & $\begin{array}{c}\% \\
\text { Amorphous }\end{array}$ & Calc. & Dolo. & Quar. & $\begin{array}{l}\text { K- } \\
\text { Feld. }\end{array}$ & Plag. & Kaol. & Mica & Chlo. & Mont. & Pyri. & Bari. & Clin. & Phil. \\
\hline 1 & 1 & $66-67$ & 89.3 & 68.5 & 00.0 & 00.0 & 31.0 & 00.0 & 11.5 & 11.2 & 42.2 & 4.0 & 00.0 & 00.0 & 00.0 & 00.0 & 00.0 \\
\hline 1 & 2 & $41-42$ & 89.9 & 70.3 & 00.0 & 00.0 & 32.7 & 00.0 & 12.1 & 9.1 & 39.2 & 6.9 & 00.0 & 00.0 & 00.0 & 00.0 & 00.0 \\
\hline 1 & 2 & $142-143$ & 90.6 & 72.4 & 00.0 & 00.0 & 25.4 & 00.0 & 13.9 & 13.2 & 42.6 & 4.8 & 00.0 & 00.0 & 00.0 & 00.0 & 00.0 \\
\hline 1 & 3 & $19-20$ & 90.3 & 71.5 & 00.0 & 00.0 & 30.1 & 00.0 & 13.2 & 8.4 & 41.0 & 7.2 & 00.0 & 00.0 & 00.0 & 00.0 & 00.0 \\
\hline 1 & 3 & $62-63$ & 90.1 & 70.9 & 00.0 & 00.0 & 32.0 & 00.0 & 11.8 & 9.0 & 41.0 & 6.2 & 00.0 & 00.0 & 00.0 & 00.0 & 00.0 \\
\hline 1 & 4 & $10-11$ & 90.9 & 73.2 & 00.0 & 00.0 & 28.4 & 00.0 & 19.3 & 00.0 & 31.8 & 20.4 & 00.0 & 00.0 & 00.0 & 00.0 & 00.0 \\
\hline 1 & 4 & $29-30$ & 90.9 & 73.2 & 00.0 & 00.0 & 26.3 & 00.0 & 19.2 & 26.3 & 21.0 & 7.1 & 00.0 & 00.0 & 00.0 & 00.0 & 00.0 \\
\hline 1 & 4 & $45-46$ & 90.5 & 72.1 & 00.0 & 00.0 & 22.5 & 00.0 & 15.3 & 00.0 & 37.8 & 8.3 & 00.0 & 00.0 & 00.0 & 00.0 & 16.0 \\
\hline 1 & 4 & $90-91$ & 90.7 & 72.6 & 00.0 & 00.0 & 25.0 & 00.0 & 37.5 & 24.0 & 00.0 & 00.0 & 00.0 & 00.0 & 00.0 & 00.0 & 13.5 \\
\hline 1 & 5 & $33-34$ & 90.5 & 72.1 & 00.0 & 00.0 & 00.0 & 00.0 & 7.3 & 23.4 & 00.0 & 00.0 & 00.0 & 00.0 & 00.0 & 00.0 & 69.3 \\
\hline 1 & 5 & $36-37$ & 90.8 & 72.9 & 00.0 & 00.0 & 10.8 & 00.0 & 2.9 & 00.0 & 23.5 & 00.0 & 00.0 & 00.0 & 00.0 & 00.0 & 62.7 \\
\hline 1 & 6 & $12-13$ & 90.2 & 71.2 & 00.0 & 00.0 & 00.0 & 00.0 & 00.0 & 00.0 & 00.0 & 00.0 & 00.0 & 00.0 & 00.0 & 00.0 & 100.0 \\
\hline 2 & 1 & $78-79$ & 91.0 & 73.5 & 00.0 & 00.0 & 00.0 & 00.0 & 12.2 & 00.0 & 00.0 & 00.0 & 00.0 & 00.0 & 00.0 & 00.0 & 87.8 \\
\hline 2 & 1 & $109-110$ & 90.0 & 70.6 & 00.0 & 00.0 & 26.0 & 00.0 & 13.0 & 12.5 & 41.5 & 7.0 & 00.0 & 00.0 & 00.0 & 00.0 & 00.0 \\
\hline 2 & 2 & $49-50$ & 91.0 & 73.5 & 00.0 & 00.0 & 21.2 & 00.0 & 35.3 & 00.0 & 00.0 & 43.5 & 00.0 & 00.0 & 00.0 & 00.0 & 00.0 \\
\hline 2 & 3 & $60-61$ & 91.0 & 100.0 & 00.0 & 00.0 & 00.0 & 00.0 & 00.0 & 00.0 & 00.0 & 00.0 & 00.0 & 00.0 & 00.0 & 00.0 & 00.0 \\
\hline 2 & 5 & $70-71$ & 91.0 & 100.0 & 00.0 & 00.0 & 00.0 & 00.0 & 00.0 & 00.0 & 00.0 & 00.0 & 00.0 & 00.0 & 00.0 & 00.0 & 00.0 \\
\hline 2 & 6 & $10-11$ & 91.0 & 100.0 & 00.0 & 00.0 & 00.0 & 00.0 & 00.0 & 00.0 & 00.0 & 00.0 & 00.0 & 00.0 & 00.0 & 00.0 & 00.0 \\
\hline 2 & 6 & $65-66$ & 91.0 & 100.0 & 00.0 & 00.0 & 00.0 & 00.0 & 00.0 & 00.0 & 00.0 & 00.0 & 00.0 & 00.0 & 00.0 & 00.0 & 00.0 \\
\hline \multicolumn{18}{|c|}{ Hole 40} \\
\hline Core & Section & $\begin{array}{l}\text { Depth } \\
\text { (cm) }\end{array}$ & Diff. & $\begin{array}{c}\% \\
\text { Amorphous }\end{array}$ & Calc. & Dolo. & Quar. & $\begin{array}{l}\text { K- } \\
\text { Feld. }\end{array}$ & Plag. & Kaol. & Mica & Chlo. & Mont. & Pyri. & Bari. & Clin. & Phil. \\
\hline 1 & 1 & $24-25$ & 90.5 & 72.1 & 00.0 & 00.0 & 29.7 & 00.0 & 17.5 & 25.8 & 26.9 & 00.0 & 00.0 & 00.0 & 00.0 & 00.0 & 00.0 \\
\hline 1 & 1 & $80-81$ & 90.5 & 72.1 & 00.0 & 00.0 & 14.9 & 00.0 & 11.3 & 7.6 & 17.0 & 00.0 & 00.0 & 00.0 & 00.0 & 00.0 & 49.1 \\
\hline 1 & 2 & $17-18$ & 90.5 & 72.1 & 00.0 & 00.0 & 16.1 & 00.0 & 1.9 & 00.0 & 25.4 & 00.0 & 00.0 & 00.0 & 00.0 & 00.0 & 56.6 \\
\hline
\end{tabular}


Hole 40 - Continued

\begin{tabular}{|c|c|c|c|c|c|c|c|c|c|c|c|c|c|c|c|c|c|}
\hline Core & Section & $\begin{array}{l}\text { Depth } \\
\text { (cm) }\end{array}$ & Diff. & $\begin{array}{c}\% \\
\text { Amorphous }\end{array}$ & Calc. & Dolo. & Quar. & $\begin{array}{l}\text { K- } \\
\text { Feld. }\end{array}$ & Plag. & Kaol. & Mica & Chlo. & Mont. & Pyri. & Bari. & Clin. & Phil. \\
\hline 1 & 2 & $75-76$ & 90.6 & 72.4 & 00.0 & 00.0 & 5.4 & 00.0 & 10.2 & 9.9 & 18.1 & 00.0 & 00.0 & 00.0 & 00.0 & 00.0 & 56.3 \\
\hline 1 & 3 & $10-11$ & 90.4 & 71.8 & 00.0 & 00.0 & 4.7 & 00.0 & 11.6 & 00.0 & 00.0 & 00.0 & 00.0 & 00.0 & 00.0 & 00.0 & 83.7 \\
\hline 1 & 3 & $75-76$ & 90.4 & 71.8 & 00.0 & 00.0 & 9.2 & 00.0 & 13.9 & 00.0 & 00.0 & 00.0 & 00.0 & 00.0 & 00.0 & 00.0 & 76.9 \\
\hline 1 & 4 & $13-14$ & 90.8 & 72.9 & 00.0 & 00.0 & 4.4 & 00.0 & 6.7 & 00.0 & 00.0 & 00.0 & 00.0 & 00.0 & 00.0 & 00.0 & 88.9 \\
\hline 1 & 5 & $10-11$ & 89.7 & 69.7 & 00.0 & 00.0 & 29.4 & 00.0 & 11.8 & 18.8 & 40.0 & 00.0 & 00.0 & 00.0 & 00.0 & 00.0 & 00.0 \\
\hline 1 & 5 & $11-12$ & 90.2 & 71.2 & 00.0 & 00.0 & 1.8 & 00.0 & 6.9 & 00.0 & 00.0 & 00.0 & 00.0 & 00.0 & 00.0 & 00.0 & 91.3 \\
\hline 1 & 5 & $76-77$ & 90.3 & 71.5 & 00.0 & 00.0 & 1.6 & 00.0 & 12.0 & 00.0 & 00.0 & 00.0 & 00.0 & 00.0 & 00.0 & 00.0 & 86.4 \\
\hline 2 & 1 & $3-4$ & 91.4 & 100.0 & 00.0 & 00.0 & 00.0 & 00.0 & 00.0 & 00.0 & 00.0 & 00.0 & 00.0 & 00.0 & 00.0 & 00.0 & 00.0 \\
\hline 2 & 1 & $73-74$ & 91.4 & 74.7 & 00.0 & 00.0 & 24.5 & 00.0 & 37.5 & 00.0 & 00.0 & 00.0 & 00.0 & 00.0 & 00.0 & 38.0 & 00.0 \\
\hline 2 & 2 & $5-6$ & 91.4 & 74.7 & 00.0 & 00.0 & 17.2 & 00.0 & 28.4 & 00.0 & 34.3 & 00.0 & 00.0 & 00.0 & 00.0 & 20.1 & 00.0 \\
\hline 2 & 3 & $10-11$ & 92.0 & 100.0 & 00.0 & 00.0 & 00.0 & 00.0 & 00.0 & 00.0 & 00.0 & 00.0 & 00.0 & 00.0 & 00.0 & 00.0 & 00.0 \\
\hline 2 & 4 & $15-16$ & 92.1 & 100.0 & 00.0 & 00.0 & 00.0 & 00.0 & 00.0 & 00.0 & 00.0 & 00.0 & 00.0 & 00.0 & 00.0 & 00.0 & 00.0 \\
\hline 2 & 5 & $109-110$ & 92.4 & 100.0 & 00.0 & 00.0 & 00.0 & 00.0 & 00.0 & 00.0 & 00.0 & 00.0 & 00.0 & 00.0 & 00.0 & 00.0 & 00.0 \\
\hline 2 & 6 & $6-7$ & 92.1 & 100.0 & 00.0 & 00.0 & 00.0 & 00.0 & 00.0 & 00.0 & 00.0 & 00.0 & 00.0 & 00.0 & 00.0 & 00.0 & 00.0 \\
\hline 3 & 2 & $13-14$ & 92.3 & 100.0 & 00.0 & 00.0 & 00.0 & 00.0 & 00.0 & 00.0 & 00.0 & 00.0 & 00.0 & 00.0 & 00.0 & 00.0 & 00.0 \\
\hline 5 & 2 & $77-78$ & 92.3 & 100.0 & 00.0 & 00.0 & 00.0 & 00.0 & 00.0 & 00.0 & 00.0 & 00.0 & 00.0 & 00.0 & 00.0 & 00.0 & 00.0 \\
\hline 5 & 3 & $10-11$ & 92.4 & 100.0 & 00.0 & 00.0 & 00.0 & 00.0 & 00.0 & 00.0 & 00.0 & 00.0 & 00.0 & 00.0 & 00.0 & 00.0 & 00.0 \\
\hline 5 & 4 & $17-18$ & 92.5 & 100.0 & 00.0 & 00.0 & 00.0 & 00.0 & 00.0 & 00.0 & 00.0 & 00.0 & 00.0 & 00.0 & 00.0 & 00.0 & 00.0 \\
\hline 5 & 5 & $10-11$ & 92.4 & 100.0 & 00.0 & 00.0 & 00.0 & 00.0 & 00.0 & 00.0 & 00.0 & 00.0 & 00.0 & 00.0 & 00.0 & 00.0 & 00.0 \\
\hline 5 & 6 & $20-21$ & 92.3 & 100.0 & 00.0 & 00.0 & 00.0 & 00.0 & 00.0 & 00.0 & 00.0 & 00.0 & 00.0 & 00.0 & 00.0 & 00.0 & 00.0 \\
\hline 6 & 1 & $11-12$ & 92.3 & 100.0 & 00.0 & 00.0 & 00.0 & 00.0 & 00.0 & 00.0 & 00.0 & 00.0 & 00.0 & 00.0 & 00.0 & 00.0 & 00.0 \\
\hline 6 & 3 & $16-18$ & 92.4 & 100.0 & 00.0 & 00.0 & 00.0 & 00.0 & 00.0 & 00.0 & 00.0 & 00.0 & 00.0 & 00.0 & 00.0 & 00.0 & 00.0 \\
\hline 6 & 4 & $10-11$ & 92.4 & 100.0 & 00.0 & 00.0 & 00.0 & 00.0 & 00.0 & 00.0 & 00.0 & 00.0 & 00.0 & 00.0 & 00.0 & 00.0 & 00.0 \\
\hline 8 & 3 & $10-11$ & 92.4 & 100.0 & 00.0 & 00.0 & 00.0 & 00.0 & 00.0 & 00.0 & 00.0 & 00.0 & 00.0 & 00.0 & 00.0 & 00.0 & 00.0 \\
\hline 9 & 2 & $10-11$ & 91.8 & 100.0 & 00.0 & 00.0 & 00.0 & 00.0 & 00.0 & 00.0 & 00.0 & 00.0 & 00.0 & 00.0 & 00.0 & 00.0 & 00.0 \\
\hline
\end{tabular}


Results of X-Ray Diffraction Analysis of Bulk Samples from Leg 5 - Continued

Hole 40 - Continued

\begin{tabular}{|c|c|c|c|c|c|c|c|c|c|c|c|c|c|c|c|c|c|}
\hline Core & Section & $\begin{array}{l}\text { Depth } \\
(\mathrm{cm})\end{array}$ & Diff. & $\begin{array}{c}\% \\
\text { Amorphous }\end{array}$ & Calc. & Dolo. & Quar. & $\begin{array}{l}\text { K- } \\
\text { Feld. }\end{array}$ & Plag. & Kaol. & Mica & Chlo. & Mont. & Pyri. & Bari. & Clin. & Phil. \\
\hline 11 & 4 & $10-11$ & 92.7 & 100.0 & 00.0 & 00.0 & 00.0 & 00.0 & 00.0 & 00.0 & 00.0 & 00.0 & 00.0 & 00.0 & 00.0 & 00.0 & 00.0 \\
\hline 13 & 1 & $100-101$ & 92.8 & 100.0 & 00.0 & 00.0 & 00.0 & 00.0 & 00.0 & 00.0 & 00.0 & 00.0 & 00.0 & 00.0 & 00.0 & 00.0 & 00.0 \\
\hline 14 & 3 & $10-11$ & 92.9 & 100.0 & 00.0 & 00.0 & 00.0 & 00.0 & 00.0 & 00.0 & 00.0 & 00.0 & 00.0 & 00.0 & 00.0 & 00.0 & 00.0 \\
\hline 15 & 2 & $50-51$ & 92.9 & 100.0 & 00.0 & 00.0 & 00.0 & 00.0 & 00.0 & 00.0 & 00.0 & 00.0 & 00.0 & 00.0 & 00.0 & 00.0 & 00.0 \\
\hline 15 & 3 & $10-11$ & 92.8 & 100.0 & 00.0 & 00.0 & 00.0 & 00.0 & 00.0 & 00.0 & 00.0 & 00.0 & 00.0 & 00.0 & 00.0 & 00.0 & 00.0 \\
\hline 15 & 4 & $10-11$ & 93.0 & 100.0 & 00.0 & 00.0 & 00.0 & 00.0 & 00.0 & 00.0 & 00.0 & 00.0 & 00.0 & 00.0 & 00.0 & 00.0 & 00.0 \\
\hline 15 & 5 & $90-91$ & 92.9 & 100.0 & 00.0 & 00.0 & 00.0 & 00.0 & 00.0 & 00.0 & 00.0 & 00.0 & 00.0 & 00.0 & 00.0 & 00.0 & 00.0 \\
\hline 15 & 6 & $33-34$ & 93.1 & 100.0 & 00.0 & 00.0 & 00.0 & 00.0 & 00.0 & 00.0 & 00.0 & 00.0 & 00.0 & 00.0 & 00.0 & 00.0 & 00.0 \\
\hline 16 & 1 & $10-11$ & 93.4 & 100.0 & 00.0 & 00.0 & 00.0 & 00.0 & 00.0 & 00.0 & 00.0 & 00.0 & 00.0 & 00.0 & 00.0 & 00.0 & 00.0 \\
\hline 16 & 3 & $85-86$ & 92.4 & 100.0 & 00.0 & 00.0 & 00.0 & 00.0 & 00.0 & 00.0 & 00.0 & 00.0 & 00.0 & 00.0 & 00.0 & 00.0 & 00.0 \\
\hline \multicolumn{18}{|c|}{ Hole 41} \\
\hline Core & Section & $\begin{array}{l}\text { Depth } \\
(\mathrm{cm})\end{array}$ & Diff. & $\begin{array}{c}\% \\
\text { Amorphous }\end{array}$ & Calc. & Dolo. & Quar. & $\begin{array}{l}\text { K- } \\
\text { Feld. }\end{array}$ & Plag. & Kaol. & Mica & Chlo. & Mont. & Pyri. & Bari. & Clin. & Phil. \\
\hline 1 & 1 & $46-47$ & 90.9 & 73.2 & 00.0 & 00.0 & 24.9 & 00.0 & 20.2 & 24.9 & 29.9 & 00.0 & 00.0 & 00.0 & 00.0 & 00.0 & 00.0 \\
\hline 1 & 2 & $10-11$ & 91.2 & 74.1 & 00.0 & 00.0 & 28.3 & 00.0 & 21.0 & 11.3 & 31.7 & 7.6 & 00.0 & 00.0 & 00.0 & 00.0 & 00.0 \\
\hline 1 & 3 & $10-11$ & 91.1 & 73.8 & 00.0 & 00.0 & 17.2 & 00.0 & 9.3 & 00.0 & 12.4 & 00.0 & 30.6 & 00.0 & 00.0 & 00.0 & 30.5 \\
\hline 1 & 4 & $16-17$ & 90.4 & 71.8 & 00.0 & 00.0 & 33.9 & 00.0 & 12.2 & 9.5 & 38.0 & 6.4 & 00.0 & 00.0 & 00.0 & 00.0 & 00.0 \\
\hline 1 & 6 & $17-18$ & 90.6 & 72.4 & 00.0 & 00.0 & 26.4 & 00.0 & 9.8 & 10.5 & 35.9 & 3.4 & 14.0 & 00.0 & 00.0 & 00.0 & 00.0 \\
\hline 2 & 1 & $10-11$ & 91.1 & 73.8 & 00.0 & 00.0 & 7.7 & 00.0 & 8.9 & 00.0 & 19.2 & 00.0 & 00.0 & 00.0 & 00.0 & 00.0 & 64.2 \\
\hline 2 & 2 & $10-11$ & 91.3 & 74.4 & 00.0 & 00.0 & 8.7 & 00.0 & 00.0 & 00.0 & 00.0 & 00.0 & 00.0 & 00.0 & 00.0 & 00.0 & 82.6 \\
\hline 2 & 3 & $10-11$ & 91.3 & 74.4 & 00.0 & 00.0 & 00.0 & 00.0 & 6.8 & 00.0 & 00.0 & 00.0 & 00.0 & 00.0 & 00.0 & 00.0 & 93.2 \\
\hline 2 & 4 & $36-37$ & 91.0 & 73.5 & 00.0 & 00.0 & 00.0 & 00.0 & 16.7 & 00.0 & 00.0 & 00.0 & 00.0 & 00.0 & 00.0 & 00.0 & 83.3 \\
\hline
\end{tabular}


Results of X-Ray Diffraction Analysis of Bulk Samples from Leg 5 - Continued

\begin{tabular}{|c|c|c|c|c|c|c|c|c|c|c|c|c|c|c|c|c|c|}
\hline Core & Section & $\begin{array}{l}\text { Depth } \\
\text { (cm) }\end{array}$ & Diff. & $\begin{array}{c}\% \\
\text { Amorphous }\end{array}$ & Calc. & Dolo. & Quar. & $\begin{array}{l}\text { K- } \\
\text { Feld. }\end{array}$ & Plag. & Kaol. & Mica & Chlo. & Mont. & Pyri. & Bari. & Clin. & Phil. \\
\hline 1 & 1 & $123-124$ & 89.8 & 70.0 & 97.5 & 00.0 & 2.5 & 00.0 & 00.0 & 00.0 & 00.0 & 00.0 & 00.0 & 00.0 & 00.0 & 00.0 & 00.0 \\
\hline 1 & 2 & $10-11$ & 89.5 & 69.1 & 98.2 & 00.0 & 1.8 & 00.0 & 00.0 & 00.0 & 00.0 & 00.0 & 00.0 & 00.0 & 00.0 & 00.0 & 00.0 \\
\hline 1 & 3 & $10-11$ & 90.3 & 71.5 & 94.1 & 00.0 & 3.4 & 00.0 & 2.5 & 00.0 & 00.0 & 00.0 & 00.0 & 00.0 & 00.0 & 00.0 & 00.0 \\
\hline 1 & 4 & $16-17$ & 89.1 & 67.9 & 97.8 & 00.0 & 2.2 & 00.0 & 00.0 & 00.0 & 00.0 & 00.0 & 00.0 & 00.0 & 00.0 & 00.0 & 00.0 \\
\hline 1 & 5 & $16-17$ & 86.5 & 60.3 & 100.0 & 00.0 & 00.0 & 00.0 & 00.0 & 00.0 & 00.0 & 00.0 & 00.0 & 00.0 & 00.0 & 00.0 & 00.0 \\
\hline 1 & 6 & $21-22$ & 86.7 & 60.9 & 100.0 & 00.0 & 00.0 & 00.0 & 00.0 & 00.0 & 00.0 & 00.0 & 00.0 & 00.0 & 00.0 & 00.0 & 00.0 \\
\hline 2 & 1 & $32-33$ & 87.4 & 62.9 & 99.2 & 00.0 & 00.0 & 00.0 & 00.0 & 00.0 & 00.0 & 00.0 & 00.0 & 00.0 & 00.0 & 00.0 & 00.0 \\
\hline 2 & 2 & $15-16$ & 85.8 & 58.2 & 100.0 & 00.0 & 00.0 & 00.0 & 00.0 & 00.0 & 00.0 & 00.0 & 00.0 & 00.0 & 00.0 & 00.0 & 00.0 \\
\hline 2 & 3 & $10-11$ & 85.9 & 58.5 & 100.0 & 00.0 & 00.0 & 00.0 & 00.0 & 00.0 & 00.0 & 00.0 & 00.0 & 00.0 & 00.0 & 00.0 & 00.0 \\
\hline 2 & 4 & $40-41$ & 87.5 & 63.2 & 99.3 & 00.0 & 00.0 & 00.0 & 00.0 & 00.0 & 00.0 & 00.0 & 00.0 & 00.0 & 00.0 & 00.0 & 00.0 \\
\hline 2 & 5 & $17-18$ & 86.8 & 61.2 & 99.9 & 00.0 & 00.0 & 00.0 & 00.0 & 00.0 & 00.0 & 00.0 & 00.0 & 00.0 & 00.0 & 00.0 & 00.0 \\
\hline 2 & 6 & $11-12$ & 88.3 & 65.6 & 98.7 & 00.0 & 1.3 & 00.0 & 00.0 & 00.0 & 00.0 & 00.0 & 00.0 & 00.0 & 00.0 & 00.0 & 00.0 \\
\hline 4 & 1 & $5-6$ & 87.2 & 62.4 & 100.0 & 00.0 & 00.0 & 00.0 & 00.0 & 00.0 & 00.0 & 00.0 & 00.0 & 00.0 & 00.0 & 00.0 & 00.0 \\
\hline 4 & 2 & $129-130$ & 88.7 & 66.8 & 99.1 & 00.0 & 00.0 & 00.0 & 00.0 & 00.0 & 00.0 & 00.0 & 00.0 & 00.0 & 00.0 & 00.0 & 00.0 \\
\hline 4 & 3 & $10-11$ & 86.1 & 59.1 & 100.0 & 00.0 & 00.0 & 00.0 & 00.0 & 00.0 & 00.0 & 00.0 & 00.0 & 00.0 & 00.0 & 00.0 & 00.0 \\
\hline 4 & 4 & $10-11$ & 91.4 & 74.7 & 91.0 & 00.0 & 3.9 & 00.0 & 5.1 & 00.0 & 00.0 & 00.0 & 00.0 & 00.0 & 00.0 & 00.0 & 00.0 \\
\hline 4 & 5 & $20-21$ & 92.6 & 78.2 & 00.0 & 00.0 & 46.1 & 00.0 & 53.9 & 00.0 & 00.0 & 00.0 & 00.0 & 00.0 & 00.0 & 00.0 & 00.0 \\
\hline 4 & 6 & $23-24$ & 92.8 & 78.8 & 00.0 & 00.0 & 100.0 & 00.0 & 00.0 & 00.0 & 00.0 & 00.0 & 00.0 & 00.0 & 00.0 & 00.0 & 00.0 \\
\hline 5 & 1 & $24-25$ & 90.6 & 72.4 & 97.8 & 00.0 & 2.2 & 00.0 & 00.0 & 00.0 & 00.0 & 00.0 & 00.0 & 00.0 & 00.0 & 00.0 & 00.0 \\
\hline 5 & 2 & $10-11$ & 92.9 & 100.0 & 00.0 & 00.0 & 00.0 & 00.0 & 00.0 & 00.0 & 00.0 & 00.0 & 00.0 & 00.0 & 00.0 & 00.0 & 00.0 \\
\hline 5 & 3 & $50-51$ & 91.2 & 74.1 & 96.9 & 00.0 & 3.1 & 00.0 & 00.0 & 00.0 & 00.0 & 00.0 & 00.0 & 00.0 & 00.0 & 00.0 & 00.0 \\
\hline 5 & 4 & $11-12$ & 91.3 & 74.4 & 00.0 & 00.0 & 47.0 & 00.0 & 53.0 & 00.0 & 00.0 & 00.0 & 00.0 & 00.0 & 00.0 & 00.0 & 00.0 \\
\hline 5 & 5 & $10-11$ & 92.4 & 77.6 & 33.2 & 00.0 & 36.9 & 00.0 & 29.9 & 00.0 & 00.0 & 00.0 & 00.0 & 00.0 & 00.0 & 00.0 & 00.0 \\
\hline 5 & 6 & $3-4$ & 92.7 & 78.5 & 00.0 & 00.0 & 100.0 & 00.0 & 00.0 & 00.0 & 00.0 & 00.0 & 00.0 & 00.0 & 00.0 & 00.0 & 00.0 \\
\hline 6 & 1 & $10-11$ & 91.5 & 75.0 & 96.3 & 00.0 & 3.7 & 00.0 & 00.0 & 00.0 & 00.0 & 00.0 & 00.0 & 00.0 & 00.0 & 00.0 & 00.0 \\
\hline
\end{tabular}


Results of X-Ray Diffraction Analysis of Bulk Samples from Leg 5 - Continued

Hole 42 - Continued

\begin{tabular}{|c|c|c|c|c|c|c|c|c|c|c|c|c|c|c|c|c|c|}
\hline Core & Section & $\begin{array}{l}\text { Depth } \\
\text { (cm) }\end{array}$ & Diff. & $\begin{array}{c}\% \\
\text { Amorphous }\end{array}$ & Calc. & Dolo. & Quar. & $\begin{array}{l}\text { K- } \\
\text { Feld. }\end{array}$ & Plag. & Kaol. & Mica & Chlo. & Mont. & Pyri. & Bari. & Clin. & Phil. \\
\hline 6 & 2 & $22-23$ & 91.7 & 75.6 & 94.7 & 00.0 & 5.3 & 00.0 & 00.0 & 00.0 & 00.0 & 00.0 & 00.0 & 00.0 & 00.0 & 00.0 & 00.0 \\
\hline 6 & 3 & $35-36$ & 91.5 & 75.0 & 96.4 & 00.0 & 3.6 & 00.0 & 00.0 & 00.0 & 00.0 & 00.0 & 00.0 & 00.0 & 00.0 & 00.0 & 00.0 \\
\hline 6 & 4 & $74-75$ & 89.6 & 69.4 & 98.8 & 00.0 & 1.2 & 00.0 & 00.0 & 00.0 & 00.0 & 00.0 & 00.0 & 00.0 & 00.0 & 00.0 & 00.0 \\
\hline 6 & 5 & $14-15$ & 90.2 & 71.2 & 98.4 & 00.0 & 1.6 & 00.0 & 00.0 & 00.0 & 00.0 & 00.0 & 00.0 & 00.0 & 00.0 & 00.0 & 00.0 \\
\hline 6 & 5 & $81-82$ & 90.2 & 71.2 & 98.5 & 00.0 & 1.5 & 00.0 & 00.0 & 00.0 & 00.0 & 00.0 & 00.0 & 00.0 & 00.0 & 00.0 & 00.0 \\
\hline 6 & 6 & $20-21$ & 93.9 & 82.1 & 100.0 & 00.0 & 00.0 & 00.0 & 00.0 & 00.0 & 00.0 & 00.0 & 00.0 & 00.0 & 00.0 & 00.0 & 00.0 \\
\hline 7 & 4 & $86-87$ & 92.0 & 100.0 & 00.0 & 00.0 & 00.0 & 00.0 & 00.0 & 00.0 & 00.0 & 00.0 & 00.0 & 00.0 & 00.0 & 00.0 & 00.0 \\
\hline 8 & 1 & $38-39$ & 87.7 & 63.8 & 100.0 & 00.0 & 00.0 & 00.0 & 00.0 & 00.0 & 00.0 & 00.0 & 00.0 & 00.0 & 00.0 & 00.0 & 00.0 \\
\hline 8 & 3 & $23-24$ & 88.2 & 65.3 & 100.0 & 00.0 & 00.0 & 00.0 & 00.0 & 00.0 & 00.0 & 00.0 & 00.0 & 00.0 & 00.0 & 00.0 & 00.0 \\
\hline 8 & 4 & $6-7$ & 87.6 & 63.5 & 100.0 & 00.0 & 00.0 & 00.0 & 00.0 & 00.0 & 00.0 & 00.0 & 00.0 & 00.0 & 00.0 & 00.0 & 00.0 \\
\hline 8 & 4 & $49-50$ & 88.8 & 67.1 & 100.0 & 00.0 & 00.0 & 00.0 & 00.0 & 00.0 & 00.0 & 00.0 & 00.0 & 00.0 & 00.0 & 00.0 & 00.0 \\
\hline 8 & 5 & $9-10$ & 88.7 & 66.8 & 100.0 & 00.0 & 00.0 & 00.0 & 00.0 & 00.0 & 00.0 & 00.0 & 00.0 & 00.0 & 00.0 & 00.0 & 00.0 \\
\hline 9 & 1 & $48-49$ & 88.1 & 65.0 & 100.0 & 00.0 & 00.0 & 00.0 & 00.0 & 00.0 & 00.0 & 00.0 & 00.0 & 00.0 & 00.0 & 00.0 & 00.0 \\
\hline 9 & 1 & $60-61$ & 91.8 & 75.9 & 100.0 & 00.0 & 00.0 & 00.0 & 00.0 & 00.0 & 00.0 & 00.0 & 00.0 & 00.0 & 00.0 & 00.0 & 00.0 \\
\hline 9 & 2 & $50-51$ & 89.8 & 70.0 & 100.0 & 00.0 & 00.0 & 00.0 & 00.0 & 00.0 & 00.0 & 00.0 & 00.0 & 00.0 & 00.0 & 00.0 & 00.0 \\
\hline 9 & 3 & $12-13$ & 91.8 & 75.9 & 100.0 & 00.0 & 00.0 & 00.0 & 00.0 & 00.0 & 00.0 & 00.0 & 00.0 & 00.0 & 00.0 & 00.0 & 00.0 \\
\hline 9 & 3 & $80-81$ & 91.9 & 76.2 & 100.0 & 00.0 & 00.0 & 00.0 & 00.0 & 00.0 & 00.0 & 00.0 & 00.0 & 00.0 & 00.0 & 00.0 & 00.0 \\
\hline 9 & 4 & $18-19$ & 89.7 & 69.7 & 100.0 & 00.0 & 00.0 & 00.0 & 00.0 & 00.0 & 00.0 & 00.0 & 00.0 & 00.0 & 00.0 & 00.0 & 00.0 \\
\hline 9 & 4 & $72-73$ & 92.1 & 100.0 & 00.0 & 00.0 & 00.0 & 00.0 & 00.0 & 00.0 & 00.0 & 00.0 & 00.0 & 00.0 & 00.0 & 00.0 & 00.0 \\
\hline 9 & 5 & $34-35$ & 90.0 & 70.6 & 100.0 & 00.0 & 00.0 & 00.0 & 00.0 & 00.0 & 00.0 & 00.0 & 00.0 & 00.0 & 00.0 & 00.0 & 00.0 \\
\hline 9 & 6 & $8-9$ & 91.3 & 74.4 & 100.0 & 00.0 & 00.0 & 00.0 & 00.0 & 00.0 & 00.0 & 00.0 & 00.0 & 00.0 & 00.0 & 00.0 & 00.0 \\
\hline 9 & 6 & $56-57$ & 90.5 & 72.1 & 100.0 & 00.0 & 00.0 & 00.0 & 00.0 & 00.0 & 00.0 & 00.0 & 00.0 & 00.0 & 00.0 & 00.0 & 00.0 \\
\hline 10 & 1 & $46-48$ & 92.2 & 100.0 & 00.0 & 00.0 & 00.0 & 00.0 & 00.0 & 00.0 & 00.0 & 00.0 & 00.0 & 00.0 & 00.0 & 00.0 & 00.0 \\
\hline 10 & 1 & $111-112$ & 91.1 & 73.8 & 100.0 & 00.0 & 00.0 & 00.0 & 00.0 & 00.0 & 00.0 & 00.0 & 00.0 & 00.0 & 00.0 & 00.0 & 00.0 \\
\hline 10 & 2 & $5-6$ & 90.1 & 70.9 & 100.0 & 00.0 & 00.0 & 00.0 & 00.0 & 00.0 & 00.0 & 00.0 & 00.0 & 00.0 & 00.0 & 00.0 & 00.0 \\
\hline
\end{tabular}


Hole 42 - Continued

\begin{tabular}{|c|c|c|c|c|c|c|c|c|c|c|c|c|c|c|c|c|c|}
\hline Core & Section & $\begin{array}{l}\text { Depth } \\
\text { (cm) }\end{array}$ & Diff. & $\begin{array}{c}\% \\
\text { Amorphous }\end{array}$ & Calc. & Dolo. & Quar. & $\begin{array}{l}\text { K- } \\
\text { Feld. }\end{array}$ & Plag. & Kaol. & Mica & Chlo. & Mont. & Pyri. & Bari. & Clin. & Phil. \\
\hline 10 & 2 & $81-82$ & 89.4 & 68.8 & 100.0 & 00.0 & 00.0 & 00.0 & 00.0 & 00.0 & 00.0 & 00.0 & 00.0 & 00.0 & 00.0 & 00.0 & 00.0 \\
\hline 10 & 3 & $18-20$ & 92.3 & 77.4 & 100.0 & 00.0 & 00.0 & 00.0 & 00.0 & 00.0 & 00.0 & 00.0 & 00.0 & 00.0 & 00.0 & 00.0 & 00.0 \\
\hline 10 & 3 & $113-114$ & 91.2 & 74.1 & 100.0 & 00.0 & 00.0 & 00.0 & 00.0 & 00.0 & 00.0 & 00.0 & 00.0 & 00.0 & 00.0 & 00.0 & 00.0 \\
\hline 10 & 4 & $4-5$ & 91.3 & 74.4 & 100.0 & 00.0 & 00.0 & 00.0 & 00.0 & 00.0 & 00.0 & 00.0 & 00.0 & 00.0 & 00.0 & 00.0 & 00.0 \\
\hline 10 & 4 & $94-95$ & 90.6 & 72.4 & 100.0 & 00.0 & 00.0 & 00.0 & 00.0 & 00.0 & 00.0 & 00.0 & 00.0 & 00.0 & 00.0 & 00.0 & 00.0 \\
\hline 10 & 6 & $14-15$ & 89.3 & 68.5 & 100.0 & 00.0 & 00.0 & 00.0 & 00.0 & 00.0 & 00.0 & 00.0 & 00.0 & 00.0 & 00.0 & 00.0 & 00.0 \\
\hline 11 & 1 & $29-30$ & 92.1 & 100.0 & 00.0 & 00.0 & 00.0 & 00.0 & 00.0 & 00.0 & 00.0 & 00.0 & 00.0 & 00.0 & 00.0 & 00.0 & 00.0 \\
\hline 11 & 2 & $48-49$ & 92.0 & 76.5 & 100.0 & 00.0 & 00.0 & 00.0 & 00.0 & 00.0 & 00.0 & 00.0 & 00.0 & 00.0 & 00.0 & 00.0 & 00.0 \\
\hline 11 & 3 & $107-108$ & 89.7 & 69.7 & 100.0 & 00.0 & 00.0 & 00.0 & 00.0 & 00.0 & 00.0 & 00.0 & 00.0 & 00.0 & 00.0 & 00.0 & 00.0 \\
\hline 11 & 5 & $27-28$ & 90.8 & 72.9 & 100.0 & 00.0 & 00.0 & 00.0 & 00.0 & 00.0 & 00.0 & 00.0 & 00.0 & 00.0 & 00.0 & 00.0 & 00.0 \\
\hline \multicolumn{18}{|c|}{ Hole 42.1} \\
\hline Core & Section & $\begin{array}{l}\text { Depth } \\
\text { (cm) }\end{array}$ & Diff. & $\begin{array}{c}\% \\
\text { Amorphous }\end{array}$ & Calc. & Dolo. & Quar. & $\begin{array}{l}\mathrm{K}- \\
\text { Feld. }\end{array}$ & Plag. & Kaol. & Mica & Chlo. & Mont. & Pyri. & Bari. & Clin. & Phil. \\
\hline 1 & 1 & $81-82$ & 89.1 & 67.9 & 100.0 & 00.0 & 00.0 & 00.0 & 00.0 & 00.0 & 00.0 & 00.0 & 00.0 & 00.0 & 00.0 & 00.0 & 00.0 \\
\hline 1 & 1 & $141-142$ & 90.3 & 71.5 & 100.0 & 00.0 & 00.0 & 00.0 & 00.0 & 00.0 & 00.0 & 00.0 & 00.0 & 00.0 & 00.0 & 00.0 & 00.0 \\
\hline 1 & 2 & $6-7$ & 90.5 & 72.1 & 100.0 & 00.0 & 00.0 & 00.0 & 00.0 & 00.0 & 00.0 & 00.0 & 00.0 & 00.0 & 00.0 & 00.0 & 00.0 \\
\hline 1 & 2 & $139-140$ & 90.2 & 71.2 & 100.0 & 00.0 & 00.0 & 00.0 & 00.0 & 00.0 & 00.0 & 00.0 & 00.0 & 00.0 & 00.0 & 00.0 & 00.0 \\
\hline 1 & 3 & $26-27$ & 92.2 & 100.0 & 00.0 & 00.0 & 00.0 & 00.0 & 00.0 & 00.0 & 00.0 & 00.0 & 00.0 & 00.0 & 00.0 & 00.0 & 00.0 \\
\hline 1 & 3 & $131-132$ & 90.1 & 70.9 & 100.0 & 00.0 & 00.0 & 00.0 & 00.0 & 00.0 & 00.0 & 00.0 & 00.0 & 00.0 & 00.0 & 00.0 & 00.0 \\
\hline 1 & 4 & $16-17$ & 91.3 & 74.4 & 100.0 & 00.0 & 00.0 & 00.0 & 00.0 & 00.0 & 00.0 & 00.0 & 00.0 & 00.0 & 00.0 & 00.0 & 00.0 \\
\hline 1 & 4 & $131-133$ & 92.1 & 100.0 & 00.0 & 00.0 & 00.0 & 00.0 & 00.0 & 00.0 & 00.0 & 00.0 & 00.0 & 00.0 & 00.0 & 00.0 & 00.0 \\
\hline 1 & 5 & $92-93$ & 92.1 & 100.0 & 00.0 & 00.0 & 00.0 & 00.0 & 00.0 & 00.0 & 00.0 & 00.0 & 00.0 & 00.0 & 00.0 & 00.0 & 00.0 \\
\hline 1 & 5 & $123-124$ & 91.4 & 74.9 & 100.0 & 00.0 & 00.0 & 00.0 & 00.0 & 00.0 & 00.0 & 00.0 & 00.0 & 00.0 & 00.0 & 00.0 & 00.0 \\
\hline
\end{tabular}


Results of X-Ray Diffraction Analysis of Bulk Samples from Leg 5 - Continued

Hole 43

\begin{tabular}{|c|c|c|c|c|c|c|c|c|c|c|c|c|c|c|c|c|c|}
\hline Core & Section & $\begin{array}{l}\text { Depth } \\
(\mathrm{cm})\end{array}$ & Diff. & $\begin{array}{c}\% \\
\text { Amorphous }\end{array}$ & Calc. & Dolo. & Quar. & $\begin{array}{l}\text { K- } \\
\text { Feld. }\end{array}$ & Plag. & Kaol. & Mica & Chlo. & Mont. & Pyri. & Bari. & Clin. & Phil. \\
\hline 1 & 2 & $95-96$ & 90.1 & 70.9 & 00.0 & 00.0 & 3.8 & 00.0 & 21.2 & 00.0 & 00.0 & 9.3 & 65.6 & 00.0 & 00.0 & 00.0 & 00.0 \\
\hline 2 & 1 & $90-91$ & 90.2 & 71.2 & 00.0 & 00.0 & 2.6 & 00.0 & 14.5 & 00.0 & 00.0 & 5.1 & 77.7 & 00.0 & 00.0 & 00.0 & 00.0 \\
\hline 2 & 2 & $10-11$ & 90.4 & 71.8 & 00.0 & 00.0 & 2.5 & 00.0 & 15.7 & 7.1 & 00.0 & 0.00 & 74.6 & 00.0 & 00.0 & 00.0 & 00.0 \\
\hline 2 & 4 & $10-11$ & 90.3 & 71.5 & 00.0 & 00.0 & 4.5 & 00.0 & 30.5 & 00.0 & 00.0 & 0.00 & 65.0 & 00.0 & 00.0 & 00.0 & 00.0 \\
\hline 2 & 5 & $100-101$ & 90.0 & 70.6 & 00.0 & 00.0 & 3.0 & 00.0 & 20.3 & 9.2 & 00.0 & 00.0 & 67.5 & 00.0 & 00.0 & 00.0 & 00.0 \\
\hline
\end{tabular}

